\title{
CONVERGENCE OF A LAGRANGE-GALERKIN METHOD FOR A FLUID-RIGID BODY SYSTEM IN ALE FORMULATION*
}

\author{
Guillaume Legendre $^{1}$ And TAKÉo TAKAhashi ${ }^{2}$
}

\begin{abstract}
We propose a numerical scheme to compute the motion of a two-dimensional rigid body in a viscous fluid. Our method combines the method of characteristics with a finite element approximation to solve an ALE formulation of the problem. We derive error estimates implying the convergence of the scheme.
\end{abstract}

Mathematics Subject Classification. 35Q30, 65M12, 76D05, 76M10.

Received April 19, 2007. Revised November 22, 2007.

Published online June 5, 2008.

\section{INTRODUCTION}

The present work aims at proposing and analyzing a Lagrange-Galerkin scheme for the numerical solution of an Arbitrary Lagrangian Eulerian (ALE) formulation of a fluid-rigid solid interaction problem. While the Lagrange-Galerkin technique has been used for years for the numerical treatment of convection-diffusion equations like the Navier-Stokes equations (see for instance $[1,26,31]$ ), it was more recently introduced in the context of ALE formulations of free surface or two-fluid flow problems $[7,12,22]$ and fluid-structure interaction problems [23,24].

The system we consider is composed of a viscous homogeneous fluid and a rigid solid, both contained in a bounded domain $\mathcal{O}$ of $\mathbb{R}^{2}$ with regular boundary $\partial \mathcal{O}$. At the initial time, the rigid body is assumed to occupy a regular open connected subset $\mathcal{S}$ of $\mathcal{O}$, surrounded by the fluid filling the domain $\mathcal{F}=\mathcal{O} \backslash \overline{\mathcal{S}}$. For the sake of simplicity and without loss of generality, we shall suppose that the center of mass of $\mathcal{S}$ is located at the origin. The domain occupied by the rigid body at each instant $t>0$ is then defined by

$$
\mathcal{S}(\boldsymbol{\zeta}(t), \theta(t))=\left\{\boldsymbol{\zeta}(t)+\mathbf{R}_{\theta(t)} \boldsymbol{x}, \boldsymbol{x} \in \mathcal{S}\right\},
$$

Keywords and phrases. Fluid-structure interaction, incompressible Navier-Stokes equations, arbitrary Lagrangian Eulerian, Lagrange-Galerkin method.

* The work of the first author was supported by FONDECYT under grant $n^{\circ} 3060018$ and by the Centro de Modelamiento Matemático.

${ }^{1}$ Centro de Modelamiento Matemático - FONDAP, UMI 2807 CNRS-Universidad de Chile, Casilla 170 - Correo 3, Santiago, Chile. glegendre@dim.uchile.cl.

Current address: CEREMADE, UMR CNRS 7534, Université de Paris-Dauphine, Place du Maréchal de Lattre de Tassigny, 75775 Paris Cedex 16, France.

${ }^{2}$ Institut de Mathématiques Élie Cartan de Nancy, Université de Nancy-CNRS-INRIA, BP 239, 54506 Vandœuvre-lès-Nancy Cedex, France. takeo.takahashi@iecn.u-nancy.fr 
where $\boldsymbol{\zeta}(t)$ and $\mathbf{R}_{\theta(t)}$ are respectively the position of the center of mass and the orientation of the rigid body at time $t\left(\mathbf{R}_{\theta}\right.$ being the matrix of rotation of angle $\left.\theta\right)$. The fluid then occupies the domain $\mathcal{F}(\boldsymbol{\zeta}(t), \theta(t))=$ $\mathcal{O} \backslash \overline{\mathcal{S}(\zeta(t), \theta(t))}$.

The fluid flow is assumed to be incompressible and modeled by the classical Navier-Stokes equations, and the motion of the rigid body governed by Newton's laws. As a consequence, the following system of partial and ordinary differential equations describes the evolution of the coupled system

$$
\begin{aligned}
& \frac{\partial \boldsymbol{u}}{\partial t}+(\boldsymbol{u} \cdot \boldsymbol{\nabla}) \boldsymbol{u}-\nu \Delta \boldsymbol{u}+\boldsymbol{\nabla} p=\boldsymbol{f} \text { in } \mathcal{F}(\boldsymbol{\zeta}(t), \theta(t)), t \in[0, T], \\
& \operatorname{div} \boldsymbol{u}=0 \text { in } \mathcal{F}(\boldsymbol{\zeta}(t), \theta(t)), t \in[0, T], \\
& \boldsymbol{u}=\mathbf{0} \text { on } \partial \mathcal{O}, t \in[0, T], \\
& \boldsymbol{u}(\boldsymbol{x}, t)=\boldsymbol{\zeta}^{\prime}(t)+\theta^{\prime}(t)(\boldsymbol{x}-\boldsymbol{\zeta}(t))^{\perp}, \boldsymbol{x} \in \partial \mathcal{S}(\boldsymbol{\zeta}(t), \theta(t)), t \in[0, T], \\
& M \boldsymbol{\zeta}^{\prime \prime}(t)=-\int_{\partial \mathcal{S}(\boldsymbol{\zeta}(t), \theta(t))} \boldsymbol{\sigma}(\boldsymbol{u}, p) \boldsymbol{n} \mathrm{d} \Gamma+\int_{\mathcal{S}(\boldsymbol{\zeta}(t), \theta(t))} \rho_{s} \boldsymbol{f}(\boldsymbol{x}, t) \mathrm{d} \boldsymbol{x}, t \in[0, T], \\
& I \theta^{\prime \prime}(t)=-\int_{\partial \mathcal{S}(\boldsymbol{\zeta}(t), \theta(t))} \boldsymbol{\sigma}(\boldsymbol{u}, p) \boldsymbol{n} \cdot(\boldsymbol{x}-\boldsymbol{\zeta}(t))^{\perp} \mathrm{d} \Gamma+\int_{\mathcal{S}(\boldsymbol{\zeta}(t), \theta(t))} \rho_{s} \boldsymbol{f}(\boldsymbol{x}, t) \cdot(\boldsymbol{x}-\boldsymbol{\zeta}(t))^{\perp} \mathrm{d} \boldsymbol{x}, t \in[0, T], \\
& \boldsymbol{u}(\cdot, 0)=\boldsymbol{u}^{(0)} \text { in } \mathcal{F}, \\
& \boldsymbol{\zeta}(0)=\mathbf{0}, \boldsymbol{\zeta}^{\prime}(0)=\boldsymbol{\zeta}^{(1)} \in \mathbb{R}^{2}, \theta(0)=0, \theta^{\prime}(0)=\theta^{(1)} \in \mathbb{R} .
\end{aligned}
$$

In the above equations, the unknowns are the Eulerian velocity field $\boldsymbol{u}(\boldsymbol{x}, t)$ and the pressure field $p(\boldsymbol{x}, t)$ in the fluid, the position $\zeta(t)$ of the center of mass and the angle of rotation $\theta(t)$ of the rigid body. To simplify, we assume that the density of the homogeneous fluid is equal to unity and that the density of the rigid body is a positive constant, denoted by $\rho_{s}$. The positive scalar $\nu$ denotes the viscosity of the fluid and $M$ and $I$ are respectively the mass and the moment of inertia of the solid. The relations between $M, I$ and $\rho_{s}$ are given by

$$
M=\int_{\mathcal{S}} \rho_{s} \mathrm{~d} \boldsymbol{x}, \quad I=\int_{\mathcal{S}} \rho_{s}|\boldsymbol{x}|^{2} \mathrm{~d} \boldsymbol{x} .
$$

The stress tensor $\boldsymbol{\sigma}$ is defined by

$$
\boldsymbol{\sigma}(\boldsymbol{u}, p)=-p \mathbf{I} \mathbf{d}+2 \nu \mathbf{D}(\boldsymbol{u}),
$$

where $\mathbf{I d}$ is the identity tensor and $\mathbf{D}(\boldsymbol{u})$ is the strain tensor given by

$$
\mathbf{D}(\boldsymbol{u})=\frac{1}{2}\left(\boldsymbol{\nabla} \boldsymbol{u}+{ }^{t} \boldsymbol{\nabla} \boldsymbol{u}\right) .
$$

Finally, the field $\boldsymbol{f}(\boldsymbol{x}, t)$ represents the density (per mass unit) of forces applied to the system, $\boldsymbol{n}$ is the unit normal vector to the boundary of the rigid body $\partial \mathcal{S}(\zeta(t), \theta(t))$, pointing into the interior of the solid and, for any $\boldsymbol{x}=\left(\begin{array}{l}x_{1} \\ x_{2}\end{array}\right)$, we have denoted by $\boldsymbol{x}^{\perp}$ the vector $\left(\begin{array}{c}-x_{2} \\ x_{1}\end{array}\right)$.

The well-posedness of this type of problem has been the subject of a large number of papers (see for instance [32] and the references given therein) and we aim at approximating strong solutions of the above system. As far as the numerical solution of such fluid-solid interaction problems is concerned, several different approaches have been introduced in the literature, based on ALE formulations [7,17,23,24], fictitious domain technique [13], penalty method [20] or Lagrange-Galerkin method [29], but only a few actually received a rigorous analysis of their properties. On this very topic, let us mention the paper of Grandmont et al. [15] for proofs of convergence of time decoupling algorithms used to solve an ALE formulation of a one-dimensional fluid-structure interaction problem. More recently, the convergence of a numerical scheme based on a Lagrange-Galerkin method, using 
a fixed mesh, has been established in [29]. Also of interest, since the present work involves a finite element approximation for solving an ALE formulation, are the paper of Gastaldi [11], which focuses on the derivation of a priori estimates in space and time in the case of an advection-diffusion equation in a moving two-dimensional domain, and the proof of convergence in [30] of a scheme based on an ALE formulation, a mixed finite element discretization in space and an implicit Euler scheme in time, for the non-steady Stokes equations in a two-dimensional, non-cylindrical domain. However, to the best of our knowledge, there is no convergence result for the numerical approximation of system (1.1)-(1.8) within an ALE framework. Our main result, stated in Theorem 3.5, asserts that the solution to a Lagrange-Galerkin discretization scheme of an ALE formulation of system (1.1)-(1.8) converges towards the exact solution of the problem, provided some assumptions on the regularity of this exact solution, on the finite element mesh and on the discrete time step. We emphasize that we managed to remove the hypothesis of a one-dimensional model [15] or of equality of the solid and fluid densities [29], which were present in the above mentioned works.

The main difficulties when studying system (1.1)-(1.8) are that the Navier-Stokes equations are coupled with some ordinary differential equations and that it defines a free boundary problem, the position of the rigid body being one of the unknowns of the problem. These issues remain at the discrete level and must be taken into consideration when devising a numerical scheme. The method presented here is characterized by the use of a semi-implicit coupling algorithm (see [9,14,27] for precisions on this terminology), in the sense that the computational domain at the next time step is obtained explicitly, by moving the nodes of the mesh with an arbitrary velocity in order to follow the motion of the rigid body, while the nonlinearities and the fluid-body coupling are treated implicitly.

Let us now discuss some of the points that contribute to make the numerical analysis in this paper technical. In spite of the fact that part of the problems encountered in establishing a convergence result can be circumvented with the help of techniques for fixed domains existing in the literature, other ones, intrinsically associated to the discretization, still have to be adequately addressed. To begin with, the notion of convergence has to be specified, since the exact and discrete solutions are defined over two different spatial domains which change with time. Their comparison will involve the essential use of a change of variables. Another important technical obstacle comes from the construction of the mesh associated to the finite element approximation of the problem. To be more precise, one would like to assume that, at least at initial time, the exact fluid domain and its approximation coincide. Without resorting to involved techniques like curved elements (whose applicability is briefly discussed in Sect. 3.3), this simple assumption requires in particular the solid body to be a polygon. As a consequence, the fluid domain is polygonal and possesses reentrant corners, which results in the exact solution of (1.1)-(1.8) not being smooth in general. Since minimal regularity is needed to establish a convergence result, we suppose that the domain is smooth and rule out the case of a boundary fitted mesh made of straight triangles. In this context, it would be natural to approximate the rigid body by a polygon which vertices are situated on the boundary of the exact body. However, this results in the approximated fluid domain not being included in the exact one and leads to a further nonconforming approximation of the fluid velocity. Dealing with such a case complicates severely the study and adds numerous technicalities that are, in our opinion, only loosely related to the free boundary aspect of the problem on which we restricted our attention. In order to keep the analysis tractable, we chose to use an approximation of the fluid domain guaranteeing the above mentioned inclusion (see Sect. 3.1). While we benefit from some previously established results on the finite element approximation, this fact yields a loss in the accuracy in space of the scheme.

An outline of the article is the following. A characteristics-ALE weak formulation of problem (1.1)-(1.8) is presented in Section 2. In Section 3, we propose a discretization scheme for this problem, describe its practical construction, and state an associated convergence theorem. The remainder of the paper, divided into four sections, is devoted to the proof of the main result: Section 4 introduces the change of variables which is the tool used for comparing the exact and approximate solutions, various preliminary error estimates for both the ALE and characteristics mappings are derived in Sections 5 and 6, and the numerical analysis of the scheme is carried out in Section 7, where the main result is finally established. 


\section{A ChARACTERISTICS-ALE FORMULATION OF THE PROBLEM}

\subsection{Hypotheses}

Throughout the paper, we shall assume that the data satisfy

$$
\begin{aligned}
& \boldsymbol{f} \in \mathrm{C}\left([0, T] ; \mathrm{H}^{1}(\mathcal{O})^{2}\right), \boldsymbol{u}^{(0)} \in \mathrm{H}^{1}(\mathcal{F})^{2}, \operatorname{div} \boldsymbol{u}^{(0)}=0 \text { in } \mathcal{F}, \\
& \boldsymbol{u}^{(0)}(\boldsymbol{x})=\boldsymbol{\zeta}^{(1)}+\theta^{(1)} \boldsymbol{x}^{\perp}, \forall \boldsymbol{x} \in \partial \mathcal{S}, \text { and } \boldsymbol{u}^{(0)}=\mathbf{0} \text { on } \partial \mathcal{O} .
\end{aligned}
$$

We moreover suppose that

$$
\operatorname{dist}(\mathcal{S}(\boldsymbol{\zeta}(t), \theta(t)), \partial \mathcal{O})>0, \forall t \in[0, T] .
$$

Owing to the result in [32], we have the following regularity for the solution to problem (1.1)-(1.8):

$$
\begin{gathered}
\boldsymbol{u} \in \mathrm{L}^{2}\left(0, T ; \mathrm{H}^{2}(\mathcal{F}(\boldsymbol{\zeta}(t), \theta(t)))^{2}\right) \cap \mathrm{H}^{1}\left(0, T ; \mathrm{L}^{2}(\mathcal{F}(\boldsymbol{\zeta}(t), \theta(t)))^{2}\right) \cap \mathrm{C}\left([0, T] ; \mathrm{H}^{1}(\mathcal{F}(\boldsymbol{\zeta}(t), \theta(t)))^{2}\right), \\
p \in \mathrm{L}^{2}\left(0, T ; \mathrm{H}^{1}(\mathcal{F}(\boldsymbol{\zeta}(t), \theta(t)))\right), \boldsymbol{\zeta} \in \mathrm{H}^{2}\left(0, T ; \mathbb{R}^{2}\right), \theta \in \mathrm{H}^{2}(0, T ; \mathbb{R}) .
\end{gathered}
$$

\subsection{Weak formulation of the problem}

For any $\boldsymbol{\zeta} \in \mathcal{O}$ and $\theta \in \mathbb{R}$ such that $\operatorname{dist}(\mathcal{S}(\boldsymbol{\zeta}, \theta), \partial \mathcal{O})>0$, we introduce the functional spaces

$$
\mathcal{V}(\boldsymbol{\zeta}, \theta)=\left\{\left(\boldsymbol{v}, \boldsymbol{\xi}_{\boldsymbol{v}}, \omega_{\boldsymbol{v}}\right) \in \mathrm{H}^{1}(\mathcal{F}(\boldsymbol{\zeta}, \theta))^{2} \times \mathbb{R}^{3} ; \boldsymbol{v}=\mathbf{0} \text { on } \partial \mathcal{O} \text { and } \boldsymbol{v}(\boldsymbol{x})=\boldsymbol{\xi}_{\boldsymbol{v}}+\omega_{\boldsymbol{v}}(\boldsymbol{x}-\boldsymbol{\zeta})^{\perp}, \forall \boldsymbol{x} \in \partial \mathcal{S}(\boldsymbol{\zeta}, \theta)\right\}
$$

and

$$
\mathcal{Q}(\boldsymbol{\zeta}, \theta)=\mathrm{L}_{0}^{2}(\mathcal{F}(\boldsymbol{\zeta}, \theta))=\left\{q \in \mathrm{L}^{2}(\mathcal{F}(\boldsymbol{\zeta}, \theta)) ; \int_{\mathcal{F}(\boldsymbol{\zeta}, \theta)} q(\boldsymbol{x}) \mathrm{d} \boldsymbol{x}=0\right\} .
$$

We denote by $\boldsymbol{\xi}=\boldsymbol{\zeta}^{\prime}$ and $\omega=\theta^{\prime}$ the translational and angular velocities of the rigid body, and use the notations

$$
\boldsymbol{f}_{\boldsymbol{M}}(t)=\int_{\mathcal{S}(\boldsymbol{\zeta}(t), \theta(t))} \rho_{s} \boldsymbol{f}(\cdot, t) \mathrm{d} \boldsymbol{x} \text { and } f_{I}(t)=\int_{\mathcal{S}(\boldsymbol{\zeta}(t), \theta(t))} \rho_{s} \boldsymbol{f}(\boldsymbol{x}, t) \cdot(\boldsymbol{x}-\boldsymbol{\zeta}(t))^{\perp} \mathrm{d} \boldsymbol{x}, \forall t \in[0, T] .
$$

One can easily check (see $[13,17,23])$ that the strong solution of $(1.1)-(1.8)$ satisfies the following mixed variational formulation: Find $(\boldsymbol{u}, \boldsymbol{\zeta}, \theta, p)$ verifying (1.7), (1.8), (2.3), and, for almost every $t$ in $(0, T)$,

$$
\begin{gathered}
\int_{\mathcal{F}(\boldsymbol{\zeta}(t), \theta(t))}\left(\frac{\partial \boldsymbol{u}}{\partial t}+(\boldsymbol{u} \cdot \boldsymbol{\nabla}) \boldsymbol{u}\right) \cdot \boldsymbol{v} \mathrm{d} \boldsymbol{x}+M \boldsymbol{\xi}^{\prime} \cdot \boldsymbol{\xi}_{\boldsymbol{v}}+I \omega^{\prime} \omega_{\boldsymbol{v}}+2 \nu \int_{\mathcal{F}(\boldsymbol{\zeta}(t), \theta(t))} \mathbf{D}(\boldsymbol{u}): \mathbf{D}(\boldsymbol{v}) \mathrm{d} \boldsymbol{x}-\int_{\mathcal{F}(\boldsymbol{\zeta}(t), \theta(t))} p \operatorname{div} \boldsymbol{v} \mathrm{d} \boldsymbol{x} \\
=\int_{\mathcal{F}(\boldsymbol{\zeta}(t), \theta(t))} \boldsymbol{f} \cdot \boldsymbol{v} \mathrm{d} \boldsymbol{x}+\boldsymbol{f}_{M} \cdot \boldsymbol{\xi}_{\boldsymbol{v}}+f_{I} \omega_{\boldsymbol{v}}, \forall\left(\boldsymbol{v}, \boldsymbol{\xi}_{\boldsymbol{v}}, \omega_{\boldsymbol{v}}\right) \in \mathcal{V}(\boldsymbol{\zeta}(t), \theta(t)), \\
-\int_{\mathcal{F}(\boldsymbol{\zeta}(t), \theta(t))} q \operatorname{div} \boldsymbol{u} \mathrm{d} \boldsymbol{x}=0, \forall q \in \mathcal{Q}(\boldsymbol{\zeta}(t), \theta(t)) .
\end{gathered}
$$

In conjunction with this weak formulation of the problem, a feature of the numerical scheme we consider is the use of the method of characteristics for the treatment of the nonlinear convection term in the Navier-Stokes equations. It is well known (see for instance [26]) that the material derivative in the flow $\boldsymbol{u}$ can be written as a total derivative

$$
\left(\frac{\partial \boldsymbol{u}}{\partial t}+(\boldsymbol{u} \cdot \boldsymbol{\nabla}) \boldsymbol{u}\right)(\boldsymbol{x}, t)=\frac{\mathrm{d}}{\mathrm{d} t}[\boldsymbol{u}(\mathcal{C}(t ; s, \boldsymbol{x}), t)]_{\left.\right|_{s=t}},
$$

by employing the characteristic function $\mathcal{C}$, which, for all $\boldsymbol{x}$ in $\mathcal{F}(\boldsymbol{\zeta}(s), \theta(s))$, is solution to the initial value problem

$$
\left\{\begin{array}{l}
\frac{\partial \mathcal{C}}{\partial t}(t ; s, \boldsymbol{x})=\boldsymbol{u}(\mathcal{C}(t ; s, \boldsymbol{x}), t) \\
\mathcal{C}(s ; s, \boldsymbol{x})=\boldsymbol{x}
\end{array}\right.
$$


These characteristics are defined over the moving domain $\mathcal{F}(\boldsymbol{\zeta}(s), \theta(s)$ ), which complicates their effective computation in a discrete setting. The idea introduced by Maury in [23,24] consists of adapting this method to an ALE framework. We address the specifics of this combination in the next subsections.

\subsection{Domain velocity and ALE mapping}

A very popular technique for the simulation of fluid-structure interaction problems since its introduction at the beginning of the eighties [6,18], the Arbitrary Lagrangian Eulerian (ALE) formulation combines advantages of both Lagrangian and Eulerian formalisms by introducing a domain velocity which makes it possible for the space discretization mesh to follow the motion of the fluid domain. Such a velocity can be defined quite arbitrarily, as long as it satisfies a compatibility condition, with respect to the fluid velocity, on the boundary of the domain $[17,22,23]$. This being done, one is able to construct a transformation linking any point of a reference configuration to a point of the current configuration, simply by using the characteristic curves associated to the domain velocity.

Choosing the fluid domain at the initial time as the frame of reference, we introduce a family of ALE mappings $\mathcal{A}(t ; 0, \cdot)$, which, at each $t$ in $[0, T]$, maps $\mathcal{F}$ into $\mathcal{F}(\boldsymbol{\zeta}(t), \theta(t))$. At each instant $t$ in $(0, T)$, it is assumed that the application $\mathcal{A}(t ; 0, \cdot)$ is an homeomorphism, that is, $\mathcal{A}(t ; 0, \cdot) \in \mathrm{C}(\overline{\mathcal{F}})^{2}$ is invertible with continuous inverse $\mathcal{A}(t ; 0, \cdot)^{-1} \in \mathrm{C}(\overline{\mathcal{F}(\boldsymbol{\zeta}(t), \theta(t))})^{2}$, and that, for all $\boldsymbol{x}$ in $\mathcal{F}$, the application $t \mapsto \mathcal{A}(t ; 0, \boldsymbol{x})$ is differentiable almost everywhere in $[0, T]$. The domain velocity $\boldsymbol{w}$ is defined by

$$
\boldsymbol{w}(\boldsymbol{x}, t)=\frac{\partial \mathcal{A}}{\partial t}\left(t ; 0, \mathcal{A}(t ; 0, \cdot)^{-1}(\boldsymbol{x})\right), \forall \boldsymbol{x} \in \mathcal{F}(\boldsymbol{\zeta}(t), \theta(t))
$$

and the ALE mapping between two time levels $s$ and $t$ in $[0, T]$ is given by

$$
\mathcal{A}(t ; s, \cdot)=\mathcal{A}(t ; 0, \cdot) \circ \mathcal{A}(s ; 0, \cdot)^{-1}
$$

It is easily seen that the application $t \mapsto \mathcal{A}(t ; s, \boldsymbol{x}), \forall \boldsymbol{x} \in \mathcal{F}(\boldsymbol{\zeta}(s), \theta(s))$, is solution to the initial value problem

$$
\left\{\begin{array}{l}
\frac{\partial \mathcal{A}}{\partial t}(t ; s, \boldsymbol{x})=\boldsymbol{w}(\mathcal{A}(t ; s, \boldsymbol{x}), t) \\
\mathcal{A}(s ; s, \boldsymbol{x})=\boldsymbol{x}
\end{array}\right.
$$

Since we will use the transformations $\mathcal{A}(t ; s, \cdot)$ in the sequel, it is important to ensure that they are compatible with the functional spaces involved in the weak formulation (2.5) of the problem. This is achieved by adding some regularity properties to the ALE mapping. Let us first recall the following classical proposition (see [16], pp. 19-20 and [10]).

Proposition 2.1. Assume that the ALE mapping $\mathcal{A}(t ; 0, \cdot)$ satisfies, for all $t$ in $(0, T)$, the following conditions:

$$
\begin{aligned}
\mathcal{F}(\boldsymbol{\zeta}(t), \theta(t))= & \mathcal{A}(t ; 0, \mathcal{F}) \text { is bounded and the boundary } \partial \mathcal{F}(\boldsymbol{\zeta}(t), \theta(t)) \text { is Lipschitz continuous, } \\
& \mathcal{A}(t ; 0, \cdot) \in \mathrm{W}^{1, \infty}(\mathcal{F})^{2}, \mathcal{A}(t ; 0, \cdot)^{-1} \in \mathrm{W}^{1, \infty}(\mathcal{F}(\boldsymbol{\zeta}(t), \theta(t)))^{2}
\end{aligned}
$$

Then, a function $v$ belongs to $\mathrm{H}^{1}(\mathcal{F}(\boldsymbol{\zeta}(t), \theta(t)))$ if and only if $\hat{v}=v \circ \mathcal{A}(t ; 0, \cdot)$ belongs to $\mathrm{H}^{1}(\mathcal{F})$.

As recalled in the references $[10,11]$, there exist several techniques in the literature to construct a mapping satisfying the above assumptions. We follow [8,11], in which the reference domain is viewed as an elastic solid being deformed into the current domain. This leads us to solve a linear elasticity problem: For all $t$ in $(0, T)$, find $d(\cdot, t)$ satisfying

$$
\left\{\begin{array}{l}
-\Delta \boldsymbol{d}(\cdot, t)-\lambda \boldsymbol{\nabla} \operatorname{div} \boldsymbol{d}(\cdot, t)=\mathbf{0} \text { in } \mathcal{F} \\
\boldsymbol{d}(\boldsymbol{x}, t)=\boldsymbol{\zeta}(t)+\mathbf{R}_{\theta(t)} \boldsymbol{x}-\boldsymbol{x} \text { on } \partial \mathcal{S} \\
\boldsymbol{d}(\cdot, t)=\mathbf{0} \text { on } \partial \mathcal{O}
\end{array}\right.
$$


where $\lambda$ is an arbitrary positive constant. Existence, uniqueness and regularity issues for solutions of this type of system have been extensively studied and it is known (see, for instance, [4,11]) that, for all $r \geqslant 2$,

$$
\|\boldsymbol{d}(\cdot, t)\|_{W^{2, r}(\mathcal{F})^{2}} \leqslant C(|\boldsymbol{\zeta}(t)|+|\theta(t)|), \forall t \in(0, T) .
$$

The ALE mapping is then defined by

$$
\mathcal{A}(t ; 0, \boldsymbol{x})=\boldsymbol{x}+\boldsymbol{d}(\boldsymbol{x}, t), \forall \boldsymbol{x} \in \mathcal{F},
$$

and we have the following result.

Lemma 2.2. Assume that

$$
\|\boldsymbol{\zeta}\|_{L^{\infty}(0, T)^{2}}+\|\theta\|_{L^{\infty}(0, T)} \leqslant c_{0}
$$

with $c_{0}$ a small enough constant. Then, for all $t$ in $(0, T)$, the mapping $\mathcal{A}(t ; 0, \cdot)$ is a diffeomorphism from $\mathcal{F}$ onto $\mathcal{F}(\boldsymbol{\zeta}(t), \theta(t))$. Moreover, it satisfies assumptions (2.12) and (2.13).

Proof. The mapping $\mathcal{A}(t ; 0, \cdot)$ is first extended to the whole of $\mathbb{R}^{2}$ by setting

$$
\boldsymbol{d}(\boldsymbol{x}, t)=\boldsymbol{\zeta}(t)+\mathbf{R}_{\theta(t)} \boldsymbol{x}-\boldsymbol{x}, \forall \boldsymbol{x} \in \mathcal{S}(\boldsymbol{\zeta}(t), \theta(t)), \text { and } \boldsymbol{d}(\cdot, t)=\mathbf{0} \text { in } \mathbb{R}^{2} \backslash \mathcal{O} .
$$

Using inequality (2.15) and assuming that the constant $c_{0}$ appearing in (2.17) is small enough, we deduce that $\boldsymbol{d}(\cdot, t)$ is a contraction. This implies the invertibility of the mapping defined by (2.16) from $\mathbb{R}^{2}$ onto $\mathbb{R}^{2}$. Since it is clear that $\mathcal{A}\left(t ; 0, \mathbb{R}^{2} \backslash \mathcal{O}\right)=\mathbb{R}^{2} \backslash \mathcal{O}$ and $\mathcal{A}(t ; 0, \mathcal{S})=\mathcal{S}(\boldsymbol{\zeta}(t), \theta(t))$, we have proved the assertion.

Remark 2.3. Assumption (2.17) is important and supposed to hold hereafter. It expresses the fact that the displacement of the rigid solid is not too large. This restriction cannot be avoided when using an ALE formulation. Indeed, as described below, the principle of this approach is to modify the mesh, according to a discrete ALE mapping, in order to follow the solid in its movement. To preserve the desired properties of the space discretization (like the regularity and quasi-uniformity of the mesh triangulation, for instance), we must assume that the displacements of the body are small.

Remark 2.4. In the proof of Lemma 2.2 , we have extended $\mathcal{A}(t ; 0, \cdot)$ to $\mathbb{R}^{2}$ and showed that it is a diffeomorphism from $\mathbb{R}^{2}$ onto $\mathbb{R}^{2}$. From now on, we will identify the mapping with its extension. Notice that it is of the form (2.16), with $\boldsymbol{d}(\cdot, t)$ small enough. More precisely, we will consider that the constant $c_{0}$ in $(2.17)$ is such that

$$
\|\boldsymbol{d}\|_{\mathrm{L}^{\infty}\left(0, T ; \mathrm{W}^{1, \infty}(\mathcal{O})^{2}\right)} \leqslant \frac{1}{4} .
$$

\subsection{Characteristics-ALE formulation}

The introduction of the ALE mapping allows us to define a new characteristic function, which involves a fixed spatial domain and is as such more manageable from a discrete point of view. The importance of this mapping comes from the fact that the material derivative in the flow can be written as a total derivative, as seen in (2.7). Let $\mathcal{B}$ be a characteristic function such that

$$
\mathcal{C}(t ; s, \boldsymbol{x})=\mathcal{A}(t ; s, \mathcal{B}(t ; s, \boldsymbol{x})), \forall \boldsymbol{x} \in \mathcal{F}(\boldsymbol{\zeta}(s), \theta(s)) .
$$

For all $t$ and $s$ in $(0, T)$, we have that the application $\mathcal{B}(t ; s, \cdot): \mathcal{F}(\boldsymbol{\zeta}(s), \theta(s)) \rightarrow \mathcal{F}(\boldsymbol{\zeta}(s), \theta(s))$ is a diffeomorphism satisfying, for all $\boldsymbol{x}$ in $\mathcal{F}(\boldsymbol{\zeta}(s), \theta(s))$, the initial value problem

$$
\left\{\begin{array}{l}
\frac{\partial \mathcal{B}}{\partial t}(t ; s, \boldsymbol{x})=(\overline{\boldsymbol{u}}-\overline{\boldsymbol{w}})(\mathcal{B}(t ; s, \boldsymbol{x}), t), \\
\mathcal{B}(s ; s, \boldsymbol{x})=\boldsymbol{x}
\end{array}\right.
$$


where the functions $\overline{\boldsymbol{u}}$ and $\overline{\boldsymbol{w}}$ are respectively defined by

$$
\overline{\boldsymbol{u}}(\boldsymbol{x}, t)=[\boldsymbol{\nabla} \mathcal{A}(t ; s, \boldsymbol{x})]^{-1} \boldsymbol{u}(\mathcal{A}(t ; s, \boldsymbol{x}), t) \text { and } \overline{\boldsymbol{w}}(\boldsymbol{x}, t)=[\boldsymbol{\nabla} \mathcal{A}(t ; s, \boldsymbol{x})]^{-1} \boldsymbol{w}(\mathcal{A}(t ; s, \boldsymbol{x}), t),
$$

for all $\boldsymbol{x}$ in $\mathcal{F}(\boldsymbol{\zeta}(s), \theta(s))$ and $t$ in $(0, T)$.

Remark 2.5. By extending the velocity field $\boldsymbol{u}(\cdot, t)$ to $\mathbb{R}^{2}$ by

$$
\boldsymbol{u}(\boldsymbol{x}, t)=\boldsymbol{\xi}(t)+\omega(t)(\boldsymbol{x}-\boldsymbol{\zeta}(t))^{\perp}, \forall \boldsymbol{x} \in \mathcal{S}(\boldsymbol{\zeta}(t), \theta(t)), \text { and } \boldsymbol{u}(\boldsymbol{x}, t)=\mathbf{0}, \forall \boldsymbol{x} \in \mathbb{R}^{2} \backslash \mathcal{O}, \forall t \in[0, T],
$$

the unique solution $\mathcal{C}(\cdot ; s, \boldsymbol{x})$ of the initial value problem $(2.8)$ exists for any $\boldsymbol{x}$ in $\mathbb{R}^{2}$. Owing to Remark 2.4, the ALE mapping $\mathcal{A}(t ; s, \cdot)$ is now defined in $\mathbb{R}^{2}$ and, consequently, so is the domain velocity $\boldsymbol{w}(\cdot, t)$. Considering these extensions, problem (2.20) actually defines a diffeomorphism $\mathcal{B}(t ; s, \cdot)$ from $\mathbb{R}^{2}$ onto $\mathbb{R}^{2}$.

Expressions (2.7) and (2.19) are finally substituted into the system (2.5)-(2.6) to yield an equivalent weak formulation of problem (1.1)-(1.8): For almost every $t$ in $(0, T)$, find $(\boldsymbol{u}, \boldsymbol{\zeta}, \theta, p)$ such that $(\boldsymbol{u}(\cdot, t), \boldsymbol{\xi}(t), \omega(t)) \in$ $\mathcal{V}(\boldsymbol{\zeta}(t), \theta(t))$ and $p(\cdot, t) \in \mathcal{Q}(\boldsymbol{\zeta}(t), \theta(t))$ are solution to

$$
\begin{aligned}
\int_{\mathcal{F}(\boldsymbol{\zeta}(t), \theta(t))} \frac{\mathrm{d}}{\mathrm{d} t}[\boldsymbol{u}(\mathcal{A}(t ; \cdot, \boldsymbol{\mathcal { B }}(t ; \cdot, \boldsymbol{x})), t)](t) \cdot \boldsymbol{v} \mathrm{d} \boldsymbol{x}+M \boldsymbol{\xi}^{\prime}(t) \cdot \boldsymbol{\xi}_{\boldsymbol{v}}+I \omega^{\prime}(t) \omega_{\boldsymbol{v}} & \\
& +2 \nu \int_{\mathcal{F}(\boldsymbol{\zeta}(t), \theta(t))} \mathbf{D}(\boldsymbol{u}): \mathbf{D}(\boldsymbol{v}) \mathrm{d} \boldsymbol{x}-\int_{\mathcal{F}(\boldsymbol{\zeta}(t), \theta(t))} p \operatorname{div} \boldsymbol{v} \mathrm{d} \boldsymbol{x} \\
= & \int_{\mathcal{F}(\boldsymbol{\zeta}(t), \theta(t))} \boldsymbol{f} \cdot \boldsymbol{v} \mathrm{d} \boldsymbol{x}+\boldsymbol{f}_{\boldsymbol{M}}(t) \cdot \boldsymbol{\xi}_{\boldsymbol{v}}+f_{I}(t) \omega_{\boldsymbol{v}}, \quad \forall\left(\boldsymbol{v}, \boldsymbol{\xi}_{\boldsymbol{v}}, \omega_{\boldsymbol{v}}\right) \in \mathcal{V}(\boldsymbol{\zeta}(t), \theta(t)) \\
& -\int_{\mathcal{F}(\boldsymbol{\zeta}(t), \theta(t))} q \operatorname{div} \boldsymbol{u} \mathrm{d} \boldsymbol{x}=0, \quad \forall q \in \mathcal{Q}(\boldsymbol{\zeta}(t), \theta(t))
\end{aligned}
$$

\section{Discretization OF THE PROBlem AND CONVERGENCE RESUlt}

This section describes the discrete scheme we propose for computing an approximation of the solution to the variational problem (2.22)-(2.23). While clearly inspired from the method introduced by Maury in [23,24] to simulate the motion of two-dimensional rigid particles in a viscous incompressible fluid, our scheme differs on two main points. First, the discrete domain velocity is derived from its associated discrete ALE mapping in a different manner. Second, for the needs of the error analysis in the convergence study, the mesh of the fluid domain must satisfy some special, non-standard features which are absent from references [23,24].

Here and subsequently, we suppose that $\mathcal{O}$ is the interior of a convex polygon. This assumption is not essential, but it allows to make simpler the forthcoming finite element analysis, while guaranteeing the expected regularity for the solution of the problem. The more general case of a domain $\mathcal{O}$ with a curved boundary $\partial \mathcal{O}$ could be dealt with by using the classical techniques presented in [5] for instance.

\subsection{Discrete scheme}

Fix $N$ in $\mathbb{N}^{*}$ and introduce a partition of the time interval $[0, T]$ by defining $t^{k}=k \delta t$ for any $k \in\{0, \ldots, N\}$, where $\delta t=T / N$. The quantities $\boldsymbol{u}_{h}^{k}, p_{h}^{k}, \boldsymbol{\zeta}_{h}^{k}, \theta_{h}^{k}, \boldsymbol{\xi}_{h}^{k}$ and $\omega_{h}^{k}$ are then the respective approximations of $\boldsymbol{u}\left(\cdot, t^{k}\right)$, $p\left(\cdot, t^{k}\right), \boldsymbol{\zeta}\left(t^{k}\right), \theta\left(t^{k}\right), \boldsymbol{\xi}\left(t^{k}\right)$ and $\omega\left(t^{k}\right)$.

\subsubsection{Initialization}

At the initial time, we consider an approximation $\mathcal{F}_{h}^{0}$ of the fluid domain $\mathcal{F}$, which is the union of straight triangles of a regular, quasi-uniform triangulation $\mathscr{T}_{h}^{0}, h$ being the discretization parameter, and satisfies the inclusion property

$$
\mathcal{F}_{h}^{0} \subset \mathcal{F}
$$




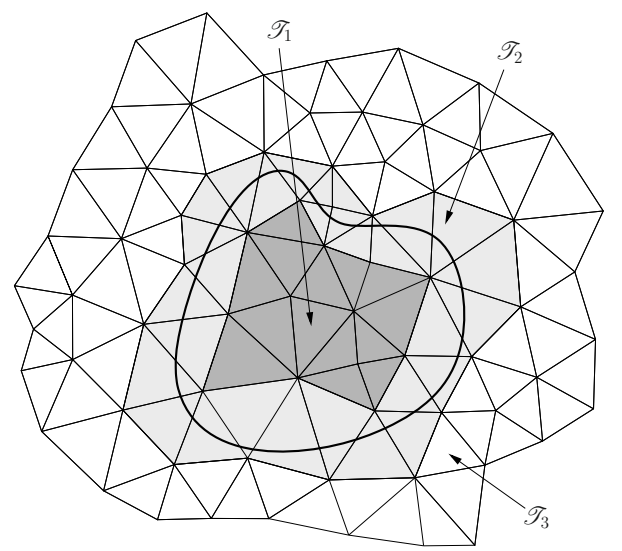

FIGURE 1. Detail of the discretization mesh with the position of the rigid solid and the three categories of triangles.

Since $\mathcal{F}$ is not convex, even if $\mathcal{O}$ is convex, hypothesis (3.1) is certainly not standard. It implies in particular that the boundary $\partial \mathcal{F}_{h}^{0}$ is a piecewise linear continuous curve whose nodes do not necessarily belong to $\partial \mathcal{F}$.

In the present work, the following process is adopted for the construction of the approximate domain ${ }^{1}$. We first build a regular, quasi-uniform triangulation $\widetilde{\mathscr{T}}_{h}^{0}$ of the whole domain $\mathcal{O}$. We then define $\mathcal{H}_{h}$, the union of all triangles in $\widetilde{\mathscr{T}}_{h}^{0}$ such that their three vertices are contained in $\overline{\mathcal{G}_{h}}$, with

$$
\mathcal{G}_{h}=\underset{\substack{K \in \widetilde{T}_{h}^{0} \\ \hdashline \cap \mathcal{S} \neq \emptyset}}{\cup} K
$$

and divide the triangles into three categories as follows (see Fig. 1):

- $\mathscr{T}_{1}$ is the subset of $\widetilde{\mathscr{T}}_{h}^{0}$ formed by all triangles $K \in \widetilde{\mathscr{T}}_{h}^{0}$ such that $\bar{K} \subset \mathcal{S}$;

- $\mathscr{T}_{2}$ is the subset formed by all triangles $K \in \widetilde{\mathscr{T}}_{h}^{0} \backslash \mathscr{T}_{1}$ such that $\bar{K} \subset \overline{\mathcal{H}_{h}}$;

- $\mathscr{T}_{3}=\widetilde{\mathscr{T}}_{h}^{0} \backslash\left(\mathscr{T}_{1} \cup \mathscr{T}_{2}\right)$.

We finally set $\mathscr{T}_{h}^{0}=\mathscr{T}_{3}$ and

$$
\mathcal{F}_{h}^{0}=\bigcup_{K \in \mathscr{T}_{h}^{0}} K, \quad \mathcal{S}_{h}^{0}=\mathcal{O} \backslash \overline{\mathcal{F}_{h}^{0}}=\bigcup_{K \in \widetilde{\mathscr{T}}_{h}^{0} \backslash \mathscr{T}_{h}^{0}} K .
$$

Notice that this approximate domain is such that it satisfies (3.1) by construction and

$$
\operatorname{dist}\left(\mathcal{F}_{h}^{0}, \mathcal{F}\right)<C h \text {. }
$$

We next define the finite element space $\mathcal{P}_{h}^{0}=\left\{\gamma \in \mathrm{C}\left(\overline{\mathcal{F}_{h}^{0}}\right)^{2} ; \gamma_{\left.\right|_{K}} \in \mathbb{P}_{1}(K), \forall K \in \mathscr{T}_{h}^{0}\right\}$, where $\mathbb{P}_{1}(K)$ denotes the set of affine functions on $K$, and its analogue $\widetilde{\mathcal{P}}_{h}^{0}=\left\{\gamma \in \mathrm{C}(\overline{\mathcal{O}})^{2} ; \gamma_{\left.\right|_{K}} \in \mathbb{P}_{1}(K), \forall K \in \widetilde{\mathscr{T}}_{h}^{0}\right\}$ over the triangulation of $\mathcal{O}$.

\footnotetext{
${ }^{1}$ It will also be the process repeated whenever a remeshing is needed (i.e., when the quality of the triangulation degrades too much due to the changes in the mesh geometry). Of course, this step, while common in practical applications of the method (see [23], in which the domain is said to be remeshed every five or ten time steps in actual computations), cannot be taken into account in the study of convergence of the scheme and we assume that the mesh remains regular enough during the whole course of its use, which is consistent with assumption (2.17).
} 
We finally take $\boldsymbol{\zeta}_{h}^{0}=\mathbf{0}, \theta_{h}^{0}=0$ and we obtain the initial approximate velocity field $\left(\boldsymbol{u}_{h}^{0}, \boldsymbol{\xi}_{h}^{0}, \omega_{h}^{0}\right)$ by first extending $\boldsymbol{u}^{(0)}$ using the rigid velocity formula

$$
\boldsymbol{u}^{(0)}(\boldsymbol{x})=\boldsymbol{\zeta}^{(1)}+\theta^{(1)} \boldsymbol{x}^{\perp}, \forall \boldsymbol{x} \in \mathcal{S},
$$

then by considering $\boldsymbol{u}_{h}^{0}$ as the projection of this extended field on $\left(\widetilde{\mathcal{P}}_{h}^{0}\right)^{2}$ and setting

$$
\boldsymbol{\xi}_{h}^{0}=\int_{\mathcal{S}_{h}^{0}} \rho_{s} \boldsymbol{u}_{h}^{0} \mathrm{~d} \boldsymbol{x} \text { and } \omega_{h}^{0}=\int_{\mathcal{S}_{h}^{0}} \rho_{s} \boldsymbol{u}_{h}^{0}(\boldsymbol{x}) \cdot\left(\boldsymbol{x}-\boldsymbol{\zeta}_{h}^{0}\right)^{\perp} \mathrm{d} \boldsymbol{x} .
$$

\subsubsection{Computation of the new domain}

Suppose that the quantities $\boldsymbol{\zeta}_{h}^{k}, \theta_{h}^{k}, \boldsymbol{\xi}_{h}^{k}$ and $\omega_{h}^{k}$ are known for some $k$ in $\{0, \ldots, N-1\}$. We approximate the position of the center of mass and the orientation of the rigid body at instant $t^{k+1}$ by

$$
\boldsymbol{\zeta}_{h}^{k+1}=\boldsymbol{\zeta}_{h}^{k}+(\delta t) \boldsymbol{\xi}_{h}^{k} \text { and } \theta_{h}^{k+1}=\theta_{h}^{k}+(\delta t) \omega_{h}^{k} .
$$

The approximations of the domains occupied respectively by the solid and fluid at instant $t^{k+1}$ are then

$$
\mathcal{S}_{h}^{k+1}=\left\{\boldsymbol{\zeta}_{h}^{k+1}+\mathbf{R}_{\theta_{h}^{k+1}-\theta_{h}^{k}}\left(\boldsymbol{x}-\boldsymbol{\zeta}_{h}^{k}\right), \boldsymbol{x} \in \mathcal{S}_{h}^{k}\right\} \text { and } \mathcal{F}_{h}^{k+1}=\mathcal{O} \backslash \overline{\mathcal{S}_{h}^{k+1}} .
$$

\subsubsection{Computation of the ALE mapping and of the characteristic function}

The finite element approximation of the ALE mapping at time $t^{k+1}$, denoted by $\mathcal{A}_{h}^{k+1}$, is defined by

$$
\mathcal{A}_{h}^{k+1}(\boldsymbol{x})=\boldsymbol{x}+\boldsymbol{d}_{h}^{k+1}(\boldsymbol{x}), \forall \boldsymbol{x} \in \mathcal{F}_{h}^{0},
$$

where the field $\boldsymbol{d}_{h}^{k+1} \in\left(\mathcal{P}_{h}^{0}\right)^{2}$ is uniquely determined by

$$
\boldsymbol{d}_{h}^{k+1}(\boldsymbol{x})=\boldsymbol{\zeta}_{h}^{k+1}+\mathbf{R}_{\theta_{h}^{k+1}} \boldsymbol{x}-\boldsymbol{x}, \forall \boldsymbol{x} \in \partial \mathcal{S}_{h}^{0}, \text { and } \boldsymbol{d}_{h}^{k+1}=\mathbf{0} \text { on } \partial \mathcal{O}
$$

and

$$
\int_{\mathcal{F}_{h}^{0}} \boldsymbol{\nabla} \boldsymbol{d}_{h}^{k+1}: \nabla \gamma_{h} \mathrm{~d} \boldsymbol{x}+\lambda \int_{\mathcal{F}_{h}^{0}}\left(\operatorname{div} \boldsymbol{d}_{h}^{k+1}\right)\left(\operatorname{div} \gamma_{h}\right) \mathrm{d} \boldsymbol{x}=0, \forall \gamma_{h} \in\left(\mathcal{P}_{h}^{0}\right)^{2} \text {, s.t. } \gamma_{h}=\mathbf{0} \text { on } \partial \mathcal{F}_{h}^{0} .
$$

Remark 3.1. Problem (3.6)-(3.7) is well-posed, but we do not know whether the mapping defined in (3.5) is invertible. Consider the field $\check{\boldsymbol{d}}_{h}^{k+1}$ solution to

$$
\left\{\begin{array}{l}
-\Delta \check{\boldsymbol{d}}_{h}^{k+1}-\lambda \boldsymbol{\nabla} \operatorname{div} \check{\boldsymbol{d}}_{h}^{k+1}=\mathbf{0} \text { in } \mathcal{F} \\
\check{\boldsymbol{d}}_{h}^{k+1}(\boldsymbol{x})=\boldsymbol{\zeta}_{h}^{k+1}+\mathbf{R}_{\theta_{h}^{k+1}} \boldsymbol{x}-\boldsymbol{x}, \forall \boldsymbol{x} \in \partial \mathcal{S} \\
\check{\boldsymbol{d}}_{h}^{k+1}=\mathbf{0} \text { on } \partial \mathcal{O}
\end{array}\right.
$$

Inequality $(4.26)$ in $[11]$ (see also $[25,28]$ ) yields the following estimate

$$
\left\|\check{\boldsymbol{d}}_{h}^{k+1}-\boldsymbol{d}_{h}^{k+1}\right\|_{\mathrm{W}^{1, \infty}\left(\mathcal{F}_{h}^{0}\right)^{2}} \leqslant C h|\log h|\left\|\check{\boldsymbol{d}}_{h}^{k+1}\right\|_{\mathrm{W}^{2, \infty}\left(\mathcal{F}_{h}^{0}\right)^{2}} .
$$

By continuity of the solution of (3.8) with respect to the data, we have

$$
\left\|\check{\boldsymbol{d}}_{h}^{k+1}\right\|_{\mathrm{W}^{2, \infty}\left(\mathcal{F}_{h}^{0}\right)^{2}} \leqslant C\left(\left|\boldsymbol{\zeta}_{h}^{k+1}\right|+\left|\theta_{h}^{k+1}\right|\right)
$$


hence

$$
\left\|\boldsymbol{d}_{h}^{k+1}\right\|_{\mathrm{W}^{1, \infty}\left(\mathcal{F}_{h}^{0}\right)^{2}} \leqslant C\left(\left|\zeta_{h}^{k+1}\right|+\left|\theta_{h}^{k+1}\right|\right)
$$

which implies, as in the continuous case and if $\left|\boldsymbol{\zeta}_{h}^{k+1}\right|+\left|\theta_{h}^{k+1}\right|$ is small enough, that $\mathcal{A}_{h}^{k+1}$ is invertible. Setting

$$
\mathcal{A}_{h}^{k+1, k}=\mathcal{A}_{h}^{k} \circ\left(\mathcal{A}_{h}^{k+1}\right)^{-1}
$$

we have in particular that $\mathcal{A}_{h}^{k+1, k}\left(\mathcal{F}_{h}^{k+1}\right)=\mathcal{F}_{h}^{k}$.

To end this remark, notice that the mapping $\mathcal{A}_{h}^{k+1}$ can be easily extended into a diffeomorphism of $\mathbb{R}^{2}$ by

$$
\boldsymbol{d}_{h}^{k+1}(\boldsymbol{x})=\boldsymbol{\zeta}_{h}^{k+1}+\mathbf{R}_{\theta_{h}^{k+1}} \boldsymbol{x}-\boldsymbol{x}, \forall \boldsymbol{x} \in \mathcal{S}_{h}^{0}, \text { and } \boldsymbol{d}_{h}^{k+1}=\mathbf{0} \text { on } \mathbb{R}^{2} \backslash \mathcal{O} .
$$

We will identify $\mathcal{A}_{h}^{k+1}$ with its extension without any change in the notation in what follows.

In order to define the approximate domain velocity $\boldsymbol{w}_{h}^{k}: \mathcal{F}_{h}^{k} \rightarrow \mathbb{R}^{2}$, we introduce the following linear interpolations in time of the approximate center of mass and orientation of the rigid solid:

$$
\theta_{h}(t)=\left(\frac{t^{k+1}-t}{\delta t}\right) \theta_{h}^{k}+\left(\frac{t-t^{k}}{\delta t}\right) \theta_{h}^{k+1} \text { and } \boldsymbol{\zeta}_{h}(t)=\left(\frac{t^{k+1}-t}{\delta t}\right) \boldsymbol{\zeta}_{h}^{k}+\left(\frac{t-t^{k}}{\delta t}\right) \boldsymbol{\zeta}_{h}^{k+1}, \forall t \in\left[t^{k}, t^{k+1}\right]
$$

and of the discrete ALE mapping:

$$
\mathcal{A}_{h}(\boldsymbol{x}, t)=\left(\frac{t^{k+1}-t}{\delta t}\right) \mathcal{A}_{h}^{k}(\boldsymbol{x})+\left(\frac{t-t^{k}}{\delta t}\right) \mathcal{A}_{h}^{k+1}(\boldsymbol{x}), \forall \boldsymbol{x} \in \mathcal{F}_{h}^{0}, \forall t \in\left[t^{k}, t^{k+1}\right] .
$$

Using (3.5) and (3.9), we infer that $\mathcal{A}_{h}(\cdot, t)$ is invertible from $\mathcal{F}_{h}^{0}$ onto $\mathcal{F}_{h}(t)=\mathcal{A}_{h}\left(\mathcal{F}_{h}^{0}, t\right)$ and set

$$
\boldsymbol{w}_{h}(\boldsymbol{x}, t)=\frac{\partial \mathcal{A}_{h}}{\partial t}\left(t, \mathcal{A}_{h}(\cdot, t)^{-1}(\boldsymbol{x})\right), \forall \boldsymbol{x} \in \mathcal{F}_{h}(t) .
$$

Introducing the finite element space $\mathcal{P}_{h}^{k}=\left\{\gamma \in \mathrm{C}\left(\overline{\mathcal{F}_{h}^{k}}\right) ; \gamma_{\left.\right|_{K}} \in \mathbb{P}_{1}(K), \forall K \in \mathscr{T}_{h}^{k}\right\}$, the approximate domain velocity at time $t^{k}, \boldsymbol{w}_{h}^{k} \in\left(\mathcal{P}_{h}^{k}\right)^{2}$, is defined as

$$
\boldsymbol{w}_{h}^{k}=\lim _{t \rightarrow t^{k}, t>t^{k}} \boldsymbol{w}_{h}(\cdot, t) .
$$

Remark 3.2. It is easily seen that, $\forall t \in\left(t^{k}, t^{k+1}\right), \boldsymbol{w}_{h}(\boldsymbol{x}, t)=\boldsymbol{\xi}_{h}^{k}+\omega_{h}^{k}\left(\boldsymbol{x}-\boldsymbol{\zeta}_{h}(t)\right)^{\perp}, \forall \boldsymbol{x} \in \partial \mathcal{S}_{h}(t)$, and $\boldsymbol{w}_{h}(\cdot, t)=\mathbf{0}$ on $\partial \mathcal{O}$, which obviously implies that $\boldsymbol{w}_{h}^{k}(\boldsymbol{x})=\boldsymbol{\xi}_{h}^{k}+\omega_{h}^{k}\left(\boldsymbol{x}-\boldsymbol{\zeta}_{\boldsymbol{h}}^{\boldsymbol{k}}\right)^{\perp}, \forall \boldsymbol{x} \in \partial \mathcal{S}_{h}^{k}$, and $\boldsymbol{w}_{h}^{k}=\mathbf{0}$ on $\partial \mathcal{O}$.

We next consider the approximate characteristic function $\mathcal{B}_{h}$ which, for all $\boldsymbol{x}$ in $\mathcal{F}_{h}^{k+1}$, is solution to

$$
\left\{\begin{array}{l}
\frac{\partial \mathcal{B}_{h}}{\partial t}\left(t ; t^{k+1}, \boldsymbol{x}\right)=\left(\overline{\boldsymbol{u}}_{\boldsymbol{h}}^{\boldsymbol{k}}-\overline{\boldsymbol{w}}_{\boldsymbol{h}}^{\boldsymbol{k}}\right)\left(\boldsymbol{\mathcal { B }}_{h}\left(t ; t^{k+1}, \boldsymbol{x}\right)\right), \\
\mathcal{B}_{h}\left(t^{k+1} ; t^{k+1}, \boldsymbol{x}\right)=\boldsymbol{x}
\end{array}\right.
$$

where

$$
\overline{\boldsymbol{u}}_{h}^{k}(\boldsymbol{x})=\left[\boldsymbol{\nabla} \mathcal{A}_{h}^{k+1, k}(\boldsymbol{x})\right]^{-1} \boldsymbol{u}_{h}^{k}\left(\mathcal{A}_{h}^{k+1, k}(\boldsymbol{x})\right) \text { and } \overline{\boldsymbol{w}}_{h}^{k}(\boldsymbol{x})=\left[\boldsymbol{\nabla} \mathcal{A}_{h}^{k+1, k}(\boldsymbol{x})\right]^{-1} \boldsymbol{w}_{h}^{k}\left(\mathcal{A}_{h}^{k+1, k}(\boldsymbol{x})\right), \forall \boldsymbol{x} \in \mathcal{F}_{h}^{k+1},
$$

and we denote $\mathcal{B}_{h}^{k}=\mathcal{B}_{h}\left(t^{k} ; t^{k+1}, \cdot\right)$.

Remark 3.3. Since the discrete ALE mappings have been extended to the whole of $\mathbb{R}^{2}$ (see Rem. 3.1), it is enough to extend the discrete velocity field $\boldsymbol{u}_{h}^{k}$ as previously done in the continuous case to define the characteristic mapping $\boldsymbol{B}_{h}^{k}$ over $\mathbb{R}^{2}$ using problem (3.14). 


\subsubsection{Calculation of the new velocity and pressure}

The triangulation $\mathscr{T}_{h}^{k+1}$ of the new domain $\mathcal{F}_{h}^{k+1}$ is obtained as the image of the triangulation $\mathscr{T}_{h}^{k}$ at the previous step by the ALE application $\mathcal{A}_{h}^{k, k+1}$. Likewise, the triangulation of $\mathcal{S}_{h}^{k+1}$ is given by $\widetilde{\mathscr{T}}_{h}^{k+1} \backslash \mathscr{T}_{h}^{k+1}$, where $\widetilde{\mathscr{T}}_{h}^{k+1}$ is the image of $\widetilde{\mathscr{T}}_{h}^{k}$ by the ALE application $\mathcal{A}_{h}^{k, k+1}$. Defining the finite element spaces

$$
\begin{aligned}
\mathcal{V}_{h}^{k+1}=\left\{\left(\boldsymbol{v}_{h}^{k+1}, \boldsymbol{\xi}_{\boldsymbol{v}_{h}^{k+1}}, \omega_{\boldsymbol{v}_{h}^{k+1}}\right) \in \mathrm{C}\left(\overline{\mathcal{F}_{h}^{k+1}}\right)^{2} \times \mathbb{R}^{3} ;\left.\boldsymbol{v}_{h}^{k+1}\right|_{\mid K} \in\left(\mathbb{P}_{1}(K) \oplus\left\langle\lambda_{1} \lambda_{2} \lambda_{3}\right\rangle\right)^{2}, \forall K \in \mathscr{T}_{h}^{k+1},\right. \\
\left.\boldsymbol{v}_{h}^{k+1}=\mathbf{0} \text { on } \partial \mathcal{O} \text { and } \boldsymbol{v}_{h}^{k+1}(\boldsymbol{x})=\boldsymbol{\xi}_{\boldsymbol{v}_{h}^{k+1}}+\omega_{\boldsymbol{v}_{h}^{k+1}}\left(\boldsymbol{x}-\boldsymbol{\zeta}_{h}^{k+1}\right)^{\perp}, \forall \boldsymbol{x} \in \partial \mathcal{S}_{h}^{k+1}\right\},
\end{aligned}
$$

with $\left\{\lambda_{i}\right\}_{i=1,2,3}$ the set of barycentric coordinates (with respect to the vertices of triangle $K$ ), and

$$
\mathcal{Q}_{h}^{k+1}=\left\{q_{h}^{k+1} \in \mathrm{C}\left(\overline{\mathcal{F}_{h}^{k+1}}\right) \cap \mathrm{L}_{0}^{2}\left(\mathcal{F}_{h}^{k+1}\right) ;\left.q_{h}^{k+1}\right|_{K} \in \mathbb{P}_{1}(K), \forall K \in \mathscr{T}_{h}^{k+1}\right\}
$$

the discrete velocity and pressure at instant $t^{k+1}$ are obtained as the solution of a discrete generalized Stokes problem: Find $\left(\boldsymbol{u}_{h}^{k+1}, \boldsymbol{\xi}_{h}^{k+1}, \omega_{h}^{k+1}\right) \in \mathcal{V}_{h}^{k+1}$ and $p_{h}^{k+1} \in \mathcal{Q}_{h}^{k+1}$ such that

$$
\begin{gathered}
\int_{\mathcal{F}_{h}^{k+1}}\left(\frac{\boldsymbol{u}_{h}^{k+1}-\boldsymbol{u}_{h}^{k} \circ \mathcal{A}_{h}^{k+1, k} \circ \mathcal{B}_{h}^{k}}{\delta t}\right) \cdot \boldsymbol{v}_{h}^{k+1} \mathrm{~d} \boldsymbol{x}+M \frac{\boldsymbol{\xi}_{h}^{k+1}-\boldsymbol{\xi}_{h}^{k}}{\delta t} \cdot \boldsymbol{\xi}_{\boldsymbol{v}_{h}^{k+1}}+I \frac{\omega_{h}^{k+1}-\omega_{h}^{k}}{\delta t} \omega_{\boldsymbol{v}_{h}^{k+1}} \\
+2 \nu \int_{\mathcal{F}_{h}^{k+1}} \mathbf{D}\left(\boldsymbol{u}_{h}^{k+1}\right): \mathbf{D}\left(\boldsymbol{v}_{h}^{k+1}\right) \mathrm{d} \boldsymbol{x}-\int_{\mathcal{F}_{h}^{k+1}} p_{h}^{k+1} \operatorname{div} \boldsymbol{v}_{h}^{k+1} \mathrm{~d} \boldsymbol{x} \\
=\int_{\mathcal{F}_{h}^{k+1}} \boldsymbol{f}_{h}^{k+1} \cdot \boldsymbol{v}_{h}^{k+1} \mathrm{~d} \boldsymbol{x}+\boldsymbol{f}_{h, \boldsymbol{M}}^{k+1} \cdot \boldsymbol{\xi}_{\boldsymbol{v}_{h}^{k+1}}+f_{h, I}^{k+1} \omega_{\boldsymbol{v}_{h}^{k+1}}, \quad \forall\left(\boldsymbol{v}_{h}^{k+1}, \boldsymbol{\xi}_{\boldsymbol{v}_{h}^{k+1}}, \omega_{\boldsymbol{v}_{h}^{k+1}}\right) \in \mathcal{V}_{h}^{k+1}, \\
-\int_{\mathcal{F}_{h}^{k+1}} q_{h}^{k+1} \operatorname{div} \boldsymbol{u}_{h}^{k+1} \mathrm{~d} \boldsymbol{x}=0, \quad \forall q_{h}^{k+1} \in \mathcal{Q}_{h}^{k+1},
\end{gathered}
$$

where $\boldsymbol{f}_{h}^{k+1}$ stands for the projection of $\boldsymbol{f}\left(\cdot, t^{k+1}\right)$ on $\left(\widetilde{\mathcal{P}}_{h}^{k+1}\right)^{2}$, with $\widetilde{\mathcal{P}}_{h}^{k+1}=\left\{\gamma \in \mathrm{C}(\overline{\mathcal{O}})^{2} ; \gamma_{\left.\right|_{K}} \in \mathbb{P}_{1}(K), \forall K \in\right.$ ${\widetilde{\mathscr{T}_{h}^{k}}}^{k}$, and

$$
\boldsymbol{f}_{h, M}^{k+1}=\int_{\mathcal{S}_{h}^{k+1}} \rho_{s} \boldsymbol{f}_{h}^{k+1} \mathrm{~d} \boldsymbol{x}, f_{h, I}^{k+1}=\int_{\mathcal{S}_{h}^{k+1}} \rho_{s} \boldsymbol{f}_{h}^{k+1}(\boldsymbol{x}) \cdot\left(\boldsymbol{x}-\boldsymbol{\zeta}_{h}^{k+1}\right)^{\perp} \mathrm{d} \boldsymbol{x} .
$$

Remark 3.4. It is worth pointing out that the discrete mixed problem (3.16)-(3.17) is well-posed for any $k \in$ $\{0, \ldots, N\}$. Indeed, by adapting the proof of Lemma 4.3 in [29], one can prove a discrete inf-sup condition, that is, there exists a positive constant $\beta_{k}$, possibly depending on $h$, such that

$$
\inf _{\boldsymbol{v}_{h} \in \mathcal{V}_{h}^{k}} \sup _{q_{h} \in \mathcal{Q}_{h}^{k}} \frac{\int_{\mathcal{F}_{h}^{k}} q_{h} \operatorname{div} \boldsymbol{v}_{h} \mathrm{~d} \boldsymbol{x}}{\left\|\boldsymbol{v}_{h}\right\|_{\mathrm{H}^{1}\left(\mathcal{F}_{h}^{k}\right)^{2}}\left\|q_{h}\right\|_{\mathrm{L}^{2}\left(\mathcal{F}_{h}^{k}\right)}} \geqslant \beta_{k} .
$$

\subsection{Statement of the main result}

Let us recall the hypotheses made so far. We have supposed that the domain $\mathcal{O}$ is the interior of a convex polygon, that there is no contact between the solid and the boundary $\partial \mathcal{O}$, a condition expressed by (2.2), and that the data verify the regularity and compatibility conditions (2.1). We shall now assume that the solution to 
problem (1.1)-(1.8) is smoother than the regularity previously given in (2.3) by making the additional hypotheses

$$
\begin{gathered}
\boldsymbol{u} \in \mathrm{C}\left([0, T] ; \mathrm{H}^{2}(\mathcal{F}(\boldsymbol{\zeta}(t), \theta(t)))^{2}\right), \frac{\mathrm{d} \boldsymbol{u}}{\mathrm{d} t} \in \mathrm{C}\left([0, T] ; \mathrm{L}^{\infty}(\mathcal{F}(\boldsymbol{\zeta}(t), \theta(t)))^{2}\right), \\
\frac{\mathrm{d}^{2} \boldsymbol{u}}{\mathrm{d} t^{2}} \in \mathrm{L}^{\infty}\left(0, T ; \mathrm{L}^{2}(\mathcal{F}(\boldsymbol{\zeta}(t), \theta(t)))^{2}\right), p \in \mathrm{C}\left([0, T] ; \mathrm{H}^{1}(\mathcal{F}(\boldsymbol{\zeta}(t), \theta(t)))\right), \\
\boldsymbol{\zeta} \in \mathrm{W}^{3, \infty}(0, T)^{2}, \omega \in \mathrm{W}^{2, \infty}(0, T),
\end{gathered}
$$

and

Our main result is the following.

$$
\boldsymbol{f} \in \mathrm{C}\left([0, T] ; \mathrm{L}^{\infty}(\mathcal{O})^{2}\right)
$$

Theorem 3.5. Assume that there exist two positive constants $c_{s}$ and $C_{s}$ such that

$$
c_{s} h^{1 / 2} \leqslant \delta t \leqslant C_{s} h^{1 / 2} .
$$

Then, under hypotheses (2.2), (2.3), (2.17), (3.18), (3.19) and the usual assumptions on the space discretization, there exist two positive constants $C$ and $\kappa$, depending on neither $h$ nor $\delta t$, such that, for all $\delta t$ in $(0, \kappa)$ and $k$ in $\{0, \ldots, N\}$, we have

and

$$
\left|\boldsymbol{\zeta}\left(t^{k}\right)-\boldsymbol{\zeta}_{h}^{k}\right|+\left|\theta\left(t^{k}\right)-\theta_{h}^{k}\right| \leqslant C(\delta t)
$$

$$
\left\|\boldsymbol{u}\left(\boldsymbol{\mathcal { A }}\left(t^{k} ; 0, \cdot\right), t^{k}\right)-\boldsymbol{u}_{h}^{k} \circ \mathcal{A}_{h}^{k}\right\|_{\mathrm{L}^{2}(\mathcal{F})^{2}}+\left|\boldsymbol{\xi}\left(t^{k}\right)-\boldsymbol{\xi}_{h}^{k}\right|+\left|\omega\left(t^{k}\right)-\omega_{h}^{k}\right| \leqslant C(\delta t) .
$$

Remark 3.6. In the above result, the ALE mappings appear in the error estimates for the velocity since the exact and approximate fields are not defined a priori in the same domain at instant $t^{k}$. Of course, we could alternatively use the extensions of these fields given in the previous sections to state a similar result without ALE mappings and quantities defined over the whole domain $\mathcal{O}$.

\subsection{Comments}

The order of convergence given in Theorem 3.5 is not as good as one would expect. Indeed, the method is shown to have an error of $O\left(\delta t+h^{1 / 2}\right)$, which is suboptimal as a piecewise linear finite element approximation is used. Moreover, this appears somewhat paradoxical since, contrary to "global" methods (like the fictitious domain formulation, the penalty technique or the Lagrange-Galerkin scheme introduced respectively in $[13,20$, 29]), the ALE formulation should allow the scheme to accurately track the motion of the rigid body. This loss in accuracy stems from the fact that the approximate domain at initial time is not based on an exact triangulation of $\mathcal{F}$. We justified this choice in the introduction by pointing out the difficulties in the analysis when using a boundary fitted mesh. In our opinion, the present paper should be viewed as a starting point for the rigorous study of more complex schemes.

An obvious extension of this work would be to employ curved finite elements for an exact (or at least fairly good) approximation of the boundary of the rigid body (see the work of Lenoir in [21]), which would hopefully give rise to a better order of convergence with respect to the space discretization parameter. Still, one should keep in mind that the no-slip condition coupling the fluid and rigid body appears in the discretization space for the fluid velocity. As a consequence, issues with the polynomial approximation of this condition at the discrete level are most likely to occur due to the mappings involved when dealing with curved simplices.

Let us finally mention that, if a polygonal rigid body is considered, the algorithm presented in these pages could certainly be worked out within the framework of singular complement methods to numerically solve the problem. This type of approximation technique relies on a decomposition of the solution into a regular part, for which our scheme provides a finite element approximation that is optimal (the domain being now exactly approximated), and a singular part, taken into account explicitly since the space of the singularities is of finite dimension. 


\subsection{Summary of the proof}

The remainder of this paper will be devoted to the proof of Theorem 3.5. Let us develop below the main ideas behind it.

First, we use the regularity of the solution to replace the time derivatives in (1.1)-(1.8) by the finite difference operators appearing in the scheme, leading to a perturbed system of semidiscretized equations which includes truncation errors. The obtained equations are next transformed according to a change of variables that maps at each time step the exact fluid domain onto the approximate one. Actually, since these two domains cannot be exactly superposed onto each other due to the space discretization, the rigid body is translated and rotated with respect to the position and orientation of his approximate counterpart. The resulting system contains variable coefficients and is thus rewritten as a system with constant coefficients and a perturbation term. This term and the errors arising from the discrepancy between the exact and approximate domains after the change of variables are then estimated.

Next, the fluid velocity and pressure are projected onto their corresponding finite element spaces. At this point, we have obtained that the projection of the solution satisfies a mixed formulation, similar to the one verified by the approximate solution, which comprises error terms emanating from the time discretization, the change of variables and the geometrical and finite element approximations. The last step consists of considering the difference between these two mixed variational formulations, taking as a test function the difference between the projected and the approximate solutions. The result is proved after some more calculations.

\section{Change of variables}

To prove Theorem 3.5, one needs to compare the exact solution, defined at instant $t^{k}$ in the domain $\mathcal{F}\left(\boldsymbol{\zeta}\left(t^{k}\right), \theta\left(t^{k}\right)\right)$, with the approximate one, defined at the same moment in the approximate domain $\mathcal{F}_{h}^{k}$. This is accomplished with the help of a change of variables similar to the one featured in [19] and subsequently used in [29] for an identical purpose. A description of this transformation and some of its properties are briefly recalled in the next subsections.

\subsection{Construction of the change of variables}

We introduce a family of changes of variable which, for any $k$ in $\{0, \ldots, N\}$, transforms a function defined on $\mathcal{F}\left(\boldsymbol{\zeta}\left(t^{k}\right), \theta\left(t^{k}\right)\right)$ into a function defined on $\mathcal{F}\left(\boldsymbol{\zeta}_{h}^{k}, \theta_{h}^{k}\right)$. First, hypotheses (2.2) and (2.3) imply the existence of $\eta>0$ such that

We then assume ${ }^{2}$ that

$$
\operatorname{dist}(\mathcal{S}(\boldsymbol{\zeta}(t), \theta(t)), \partial \mathcal{O})>\eta, \forall t \in[0, T] .
$$

$$
\left|\boldsymbol{\zeta}\left(t^{k}\right)-\boldsymbol{\zeta}_{h}^{k}\right|+\left|\theta\left(t^{k}\right)-\theta_{h}^{k}\right| \leqslant C(\delta t)
$$

for some positive constant $C$ independent of $h$ and $k$. As a consequence, for $\delta t$ and $h$ both small enough, we verify that, for all $\lambda$ in $[0,1]$,

$$
\lambda \boldsymbol{\zeta}\left(t^{k}\right)+(1-\lambda) \boldsymbol{\zeta}_{h}^{k}+\mathbf{R}_{\lambda\left(\theta\left(t^{k}\right)-\theta_{h}^{k}\right)}\left(\mathcal{S}\left(\boldsymbol{\zeta}_{h}^{k}, \theta_{h}^{k}\right)-\boldsymbol{\zeta}_{h}^{k}\right) \subset \mathcal{O},
$$

and

$$
\operatorname{dist}\left(\lambda \boldsymbol{\zeta}\left(t^{k}\right)+(1-\lambda) \boldsymbol{\zeta}_{h}^{k}+\mathbf{R}_{\lambda\left(\theta\left(t^{k}\right)-\theta_{h}^{k}\right)}\left(\mathcal{S}\left(\boldsymbol{\zeta}_{h}^{k}, \theta_{h}^{k}\right)-\boldsymbol{\zeta}_{h}^{k}\right), \partial \mathcal{O}\right)>\frac{\eta}{2} .
$$

Let $\chi \in \mathrm{C}^{\infty}\left(\mathbb{R}^{2} ; \mathbb{R}\right)$ be a function with compact support contained in $\mathcal{O}$, such that, for all $\boldsymbol{x}$ in $\mathcal{O}$,

$$
\chi(\boldsymbol{x})= \begin{cases}1 & \text { if } \operatorname{dist}(\boldsymbol{x}, \partial \mathcal{O})>\frac{\eta}{2}, \\ 0 & \text { if } \operatorname{dist}(\boldsymbol{x}, \partial \mathcal{O})<\frac{\eta}{4} .\end{cases}
$$

\footnotetext{
${ }^{2}$ This assumption will be proved later by induction.
} 
Defining the following smooth functions for all $\boldsymbol{x}$ in $\mathcal{O}$ and all $\lambda$ in $[0,1]$

$\tau^{k}(\boldsymbol{x}, \lambda)=\left(\boldsymbol{\zeta}\left(t^{k}\right)-\boldsymbol{\zeta}_{h}^{k}\right) \cdot \boldsymbol{x}^{\perp}+\left(\theta\left(t^{k}\right)-\theta_{h}^{k}\right)\left(\frac{|\boldsymbol{x}|^{2}}{2}-\left(\lambda \boldsymbol{\zeta}\left(t^{k}\right)+(1-\lambda) \boldsymbol{\zeta}_{h}^{k}\right) \cdot \boldsymbol{x}\right)$ and $\boldsymbol{\Lambda}^{k}(\boldsymbol{x}, \lambda)=\boldsymbol{\nabla}\left[\chi(\boldsymbol{x}) \tau^{k}(\boldsymbol{x}, \lambda)\right]^{\perp}$,

we check that

$$
\boldsymbol{\Lambda}^{k}(\boldsymbol{x}, \lambda)= \begin{cases}\boldsymbol{\zeta}\left(t^{k}\right)-\boldsymbol{\zeta}_{h}^{k}+\left(\theta\left(t^{k}\right)-\theta_{h}^{k}\right)\left(\boldsymbol{x}-\lambda \boldsymbol{\zeta}\left(t^{k}\right)-(1-\lambda) \boldsymbol{\zeta}_{h}^{k}\right)^{\perp} & \text { if } \operatorname{dist}(\boldsymbol{x}, \partial \mathcal{O})>\frac{\eta}{2} \\ \mathbf{0} & \text { if } \operatorname{dist}(\boldsymbol{x}, \partial \mathcal{O})<\frac{\eta}{4}\end{cases}
$$

and

$$
\operatorname{div} \boldsymbol{\Lambda}^{k}(\boldsymbol{x}, \lambda)=0
$$

Considering the following initial value problem

$$
\left\{\begin{array}{l}
\frac{\partial \psi}{\partial \lambda}(\boldsymbol{y}, \lambda)=\boldsymbol{\Lambda}^{k}(\boldsymbol{\psi}(\boldsymbol{y}, \lambda), \lambda) \\
\boldsymbol{\psi}(\boldsymbol{y}, 0)=\boldsymbol{y}, \boldsymbol{y} \in \mathcal{O}
\end{array}\right.
$$

we can show that the application

$$
\mathbf{X}^{k}(\boldsymbol{y})=\boldsymbol{\psi}(\boldsymbol{y}, 1), \forall \boldsymbol{y} \in \mathcal{F}\left(\boldsymbol{\zeta}_{h}^{k}, \theta_{h}^{k}\right),
$$

is a diffeomorphism which maps $\mathcal{F}\left(\boldsymbol{\zeta}_{h}^{k}, \theta_{h}^{k}\right)$ onto $\mathcal{F}\left(\boldsymbol{\zeta}\left(t^{k}\right), \theta\left(t^{k}\right)\right)$ and satisfies

$$
\mathbf{X}^{k}(\boldsymbol{y})=\boldsymbol{\zeta}\left(t^{k}\right)+\mathbf{R}_{\theta\left(t^{k}\right)-\theta_{h}^{k}}\left(\boldsymbol{y}-\boldsymbol{\zeta}_{h}^{k}\right)
$$

for any $\boldsymbol{y}$ in a neighborhood of $\partial \mathcal{S}\left(\boldsymbol{\zeta}_{h}^{k}, \theta_{h}^{k}\right)$.

\subsection{Transformed system}

The purpose of the change of variables defined by the mapping $\mathbf{X}^{k}$ is to be applied to the exact solution of problem (1.1)-(1.8). We thus introduce the following quantities

$$
\begin{gathered}
\mathbf{U}^{k}(\boldsymbol{y})=J_{\mathbf{Y}^{k}}\left(\mathbf{X}^{k}(\boldsymbol{y})\right) \boldsymbol{u}\left(\mathbf{X}^{k}(\boldsymbol{y}), t^{k}\right), \mathrm{P}^{k}(\boldsymbol{y})=p\left(\mathbf{X}^{k}(\boldsymbol{y}), t^{k}\right), \forall \boldsymbol{y} \in \mathcal{F}\left(\boldsymbol{\zeta}_{h}^{k}, \theta_{h}^{k}\right), \\
\boldsymbol{\Xi}^{k}=\mathbf{R}_{\theta_{h}^{k}-\theta\left(t^{k}\right)} \boldsymbol{\xi}\left(t^{k}\right) \text { and } \Omega^{k}=\omega\left(t^{k}\right),
\end{gathered}
$$

where we have denoted by $\mathbf{Y}^{k}$ the inverse of $\mathbf{X}^{k}$ and by $J_{\mathbf{Y}^{k}}$ the Jacobian matrix of $\mathbf{Y}^{k}$

$$
J_{\mathbf{Y}^{k}}=\left(\frac{\partial Y^{k}{ }_{i}}{\partial x_{j}}\right)_{1 \leqslant i, j \leqslant 2} .
$$

Also needed will be the transformed characteristic function

$$
\mathcal{C}^{k}=\mathbf{Y}^{k} \circ \mathcal{C}\left(t^{k} ; t^{k+1}, \cdot\right) \circ \mathbf{X}^{k+1} .
$$

This change of variables is inspired by the work of Inoue and Wakimoto in [19]. In particular, the fact that the field $\boldsymbol{\Lambda}^{k}$ has a vanishing divergence yields the conservation of the divergence of the fluid velocity field through the transformation. More precisely, owing to a Liouville lemma (see for instance [2], p. 251) and property (4.2), we have

$$
\operatorname{div} \mathbf{U}^{k}=0 \text { in } \mathcal{F}\left(\boldsymbol{\zeta}_{h}^{k}, \theta_{h}^{k}\right),
$$


and, using notably (4.4), we verify that

$$
\mathbf{U}^{k}(\boldsymbol{y})=\boldsymbol{\Xi}^{k}+\Omega^{k}\left(\boldsymbol{y}-\boldsymbol{\zeta}_{h}^{k}\right)^{\perp}, \forall \boldsymbol{y} \in \partial \mathcal{S}\left(\boldsymbol{\zeta}_{h}^{k}, \theta_{h}^{k}\right), \text { and } \mathbf{U}^{k}=\mathbf{0} \text { on } \partial \mathcal{O} .
$$

Finally, in order to write down the expressions of $\Delta \boldsymbol{u}$ and $\boldsymbol{\nabla} p$ after the change of variables, we define (see [19])

$$
\begin{gathered}
{\left[L^{k} \mathbf{U}^{k}\right]_{i}=\sum_{j, l=1}^{2} \frac{\partial}{\partial y_{j}}\left(g^{j l} \frac{\partial \mathrm{U}^{k}}{\partial y_{l}}\right)+2 \sum_{j, l, m=1}^{2} g^{l m} \Gamma_{j l}^{i} \frac{\partial \mathrm{U}_{j}^{k}}{\partial y_{m}}+\sum_{j, l, m=1}^{2}\left(\frac{\partial}{\partial y_{l}}\left(g^{l m} \Gamma_{j m}^{i}\right)+\sum_{n=1}^{2} g^{l m} \Gamma_{j m}^{n} \Gamma_{l n}^{i}\right) \mathrm{U}_{j}^{k},} \\
{\left[\boldsymbol{G}^{k} \mathrm{P}^{k}\right]_{i}=\sum_{j=1}^{2} g^{i j} \frac{\partial \mathrm{P}^{k}}{\partial y_{j}}} \\
g^{i j}=\sum_{l=1}^{2} \frac{\partial \mathrm{Y}_{i}^{k}}{\partial x_{l}} \frac{\partial \mathrm{Y}_{j}^{k}}{\partial x_{l}}, g_{i j}=\sum_{l=1}^{2} \frac{\partial \mathrm{X}_{i}^{k}}{\partial y_{l}} \frac{\partial \mathrm{X}_{j}^{k}}{\partial y_{l}} \\
\Gamma_{i j}^{l}=\frac{1}{2} \sum_{m=1}^{2} g^{l m}\left(\frac{\partial g_{i m}}{\partial y_{j}}+\frac{\partial g_{j m}}{\partial y_{i}}+\frac{\partial g_{i j}}{\partial y_{m}}\right)
\end{gathered}
$$

\subsection{Properties}

We end this section by stating a series of estimates satisfied by the change of variables. Their proofs can be obtained from [32] and [29], where the same kind of estimates are derived.

All the letters $C$ appearing throughout the paper will denote generic, strictly positive constants which are independent of $\delta t, h$ and $k$.

Lemma 4.1. The function $\mathbf{X}^{k}$, defined by (4.3), and its inverse $\mathbf{Y}^{k}$ satisfy the following inequalities

$$
\begin{gathered}
\left\|\mathbf{X}^{k}\right\|_{\mathrm{L}^{\infty}\left(\mathcal{F}\left(\boldsymbol{\zeta}_{h}^{k}, \theta_{h}^{k}\right)\right)^{2}} \leqslant C,\left\|\mathbf{Y}^{k}\right\|_{\mathrm{L}^{\infty}\left(\mathcal{F}\left(\boldsymbol{\zeta}\left(t^{k}\right), \theta\left(t^{k}\right)\right)\right)^{2}} \leqslant C \\
\left\|\mathbf{X}^{k}-\mathbf{I d}\right\|_{\mathrm{L}^{\infty}(\mathcal{O})^{2}} \leqslant C\left(\left|\boldsymbol{\zeta}\left(t^{k}\right)-\boldsymbol{\zeta}_{h}^{k}\right|+\left|\theta\left(t^{k}\right)-\theta_{h}^{k}\right|\right),\left\|\mathbf{Y}^{k}-\mathbf{I d}\right\|_{\mathrm{L}^{\infty}(\mathcal{O})^{2}} \leqslant C\left(\left|\boldsymbol{\zeta}\left(t^{k}\right)-\boldsymbol{\zeta}_{h}^{k}\right|+\left|\theta\left(t^{k}\right)-\theta_{h}^{k}\right|\right), \\
\left\|J_{\mathbf{X}^{k}}-\mathbf{I d}\right\|_{\mathrm{L}^{\infty}(\mathcal{O})^{4}} \leqslant C\left(\left|\boldsymbol{\zeta}\left(t^{k}\right)-\boldsymbol{\zeta}_{h}^{k}\right|+\left|\theta\left(t^{k}\right)-\theta_{h}^{k}\right|\right),\left\|J_{\mathbf{Y}^{k}}-\mathbf{I d}\right\|_{\mathrm{L}^{\infty}(\mathcal{O})^{4}} \leqslant C\left(\left|\boldsymbol{\zeta}\left(t^{k}\right)-\boldsymbol{\zeta}_{h}^{k}\right|+\left|\theta\left(t^{k}\right)-\theta_{h}^{k}\right|\right) \\
\left\|\frac{\partial^{2} \mathbf{X}^{k}}{\partial y_{i} \partial y_{j}}\right\|_{L^{\infty}(\mathcal{O})^{2}} \leqslant C\left(\left|\boldsymbol{\zeta}\left(t^{k}\right)-\boldsymbol{\zeta}_{h}^{k}\right|+\left|\theta\left(t^{k}\right)-\theta_{h}^{k}\right|\right),\left\|\frac{\partial^{2} \mathbf{Y}^{k}}{\partial x_{i} \partial x_{j}}\right\|_{L^{\infty}(\mathcal{O})^{2}} \leqslant C\left(\left|\boldsymbol{\zeta}\left(t^{k}\right)-\boldsymbol{\zeta}_{h}^{k}\right|+\left|\theta\left(t^{k}\right)-\theta_{h}^{k}\right|\right),
\end{gathered}
$$

Lemma 4.2. Assume that $\left(\mathbf{U}^{k}, \mathrm{P}^{k}\right) \in \mathrm{H}^{2}\left(\mathcal{F}\left(\boldsymbol{\zeta}_{h}^{k}, \theta_{h}^{k}\right)\right)^{2} \times \mathrm{H}^{1}\left(\mathcal{F}\left(\boldsymbol{\zeta}_{h}^{k}, \theta_{h}^{k}\right)\right)$. We have

$$
\begin{gathered}
\left\|\nu\left(L^{k}-\Delta\right) \mathbf{U}^{k}\right\|_{L^{2}\left(\mathcal{F}\left(\boldsymbol{\zeta}_{h}^{k}, \theta_{h}^{k}\right)\right)^{2}} \leqslant C\left(\left|\boldsymbol{\zeta}\left(t^{k}\right)-\boldsymbol{\zeta}_{h}^{k}\right|+\left|\theta\left(t^{k}\right)-\theta_{h}^{k}\right|\right)\left\|\mathbf{U}^{k}\right\|_{\mathrm{H}^{2}\left(\mathcal{F}\left(\boldsymbol{\zeta}_{h}^{k}, \theta_{h}^{k}\right)\right)^{2}}, \\
\left\|\left(\boldsymbol{\nabla}-\boldsymbol{G}^{k}\right) \mathrm{P}^{k}\right\|_{\mathrm{L}^{2}\left(\mathcal{F}\left(\boldsymbol{\zeta}_{h}^{k}, \theta_{h}^{k}\right)\right)^{2}} \leqslant C\left(\left|\boldsymbol{\zeta}\left(t^{k}\right)-\boldsymbol{\zeta}_{h}^{k}\right|+\left|\theta\left(t^{k}\right)-\theta_{h}^{k}\right|\right)\left\|\mathrm{P}^{k}\right\|_{\mathrm{H}^{1}\left(\mathcal{F}\left(\boldsymbol{\zeta}_{h}^{k}, \theta_{h}^{k}\right)\right)} .
\end{gathered}
$$

Lemma 4.3. The function $\mathbf{X}^{k}$, defined by (4.3), and its inverse $\mathbf{Y}^{k}$ satisfy the following inequalities

$$
\begin{aligned}
& \left\|\mathbf{X}^{k+1}-\mathbf{X}^{k}\right\|_{\mathrm{L}^{\infty}(\mathcal{O})^{2}} \leqslant C(\delta t)\left(\left|\theta\left(t^{k}\right)-\theta_{h}^{k}\right|+\left|\boldsymbol{\xi}\left(t^{k}\right)-\boldsymbol{\xi}_{h}^{k}\right|+\left|\omega\left(t^{k}\right)-\omega_{h}^{k}\right|+\delta t\right), \\
& \left\|\mathbf{Y}^{k+1}-\mathbf{Y}^{k}\right\|_{L^{\infty}(\mathcal{O})^{2}} \leqslant C(\delta t)\left(\left|\theta\left(t^{k}\right)-\theta_{h}^{k}\right|+\left|\boldsymbol{\xi}\left(t^{k}\right)-\boldsymbol{\xi}_{h}^{k}\right|+\left|\omega\left(t^{k}\right)-\omega_{h}^{k}\right|+\delta t\right), \\
& \left\|J_{\mathbf{X}^{k+1}}-J_{\mathbf{X}^{k}}\right\|_{\mathrm{L}^{\infty}(\mathcal{O})^{4}} \leqslant C(\delta t)\left(\left|\theta\left(t^{k}\right)-\theta_{h}^{k}\right|+\left|\boldsymbol{\xi}\left(t^{k}\right)-\boldsymbol{\xi}_{h}^{k}\right|+\left|\omega\left(t^{k}\right)-\omega_{h}^{k}\right|+\delta t\right) .
\end{aligned}
$$




\section{ERror Bounds on the ALE MAPPINGS}

A number of preliminary results related to the ALE mappings are established in this section. As previously, it is assumed that (4.1) holds and that $\delta t$ and $h$ are small enough, so that the preceding results are valid. We also suppose that $\delta t$ and $h$ are both smaller than unity to simplify the estimates.

We first complete Lemma 2.2 by giving additional results on the continuous ALE mapping. By differentiating problem $(2.14)$ with respect to time, we obtain that $\frac{\partial \boldsymbol{d}}{\partial t}(\cdot, t)$ is solution of the system

$$
\left\{\begin{array}{l}
-\Delta\left(\frac{\partial \boldsymbol{d}}{\partial t}\right)(\cdot, t)-\lambda \boldsymbol{\nabla} \operatorname{div}\left(\frac{\partial \boldsymbol{d}}{\partial t}\right)(\cdot, t)=\mathbf{0} \text { in } \mathcal{F} \\
\left(\frac{\partial \boldsymbol{d}}{\partial t}\right)(\boldsymbol{x}, t)=\boldsymbol{\xi}(t)+\omega(t) \mathbf{R}_{\theta(t)} \boldsymbol{x}^{\perp}, \forall \boldsymbol{x} \in \partial \mathcal{S} \\
\left(\frac{\partial \boldsymbol{d}}{\partial t}\right)(\cdot, t)=\mathbf{0} \text { on } \partial \mathcal{O}
\end{array}\right.
$$

In particular, the following estimate holds (see for instance [4,11])

$$
\left\|\frac{\partial \boldsymbol{d}}{\partial t}(\cdot, t)\right\|_{W^{2, \infty}(\mathcal{F})^{2}} \leqslant C(|\boldsymbol{\xi}(t)|+|\omega(t)|), \forall t \in(0, T) .
$$

Using regularity assumptions (2.1), we conclude that

$$
\boldsymbol{d}, \boldsymbol{A}(\cdot ; 0, \cdot) \in \mathrm{W}^{2, \infty}\left(0, T ; \mathrm{W}^{2, \infty}(\mathcal{F})^{2}\right)
$$

One also has, owing to definition (2.9),

$$
\boldsymbol{w} \in \mathrm{W}^{1, \infty}\left(0, T ; \mathrm{W}^{2, \infty}(\mathcal{F})^{2}\right)
$$

and, from the proof of Lemma 2.2,

$$
(\boldsymbol{x}, t) \mapsto(\mathcal{A}(t ; 0, \cdot))^{-1}(\boldsymbol{x}) \in \mathrm{W}^{2, \infty}\left(0, T ; \mathrm{W}^{2, \infty}(\mathcal{F}(\boldsymbol{\zeta}(t), \theta(t)))^{2}\right) .
$$

We now give an estimate on the difference between the mapping $\mathcal{A}\left(t^{k} ; 0, \cdot\right)$ and its discrete counterpart $\mathcal{A}_{h}^{k}$. Recall that both of these mappings have been extended (see Rems. 2.4 and 3.1) to the whole of $\mathbb{R}^{2}$.

Lemma 5.1. There exists a constant $C$ such that, for all $k \in\{0, \ldots, N\}$,

$$
\begin{gathered}
\left\|\boldsymbol{A}\left(t^{k} ; 0, \cdot\right)-\mathcal{A}_{h}^{k}\right\|_{L^{\infty}(\mathcal{O})^{2}} \leqslant C\left(\left|\boldsymbol{\zeta}\left(t^{k}\right)-\boldsymbol{\zeta}_{h}^{k}\right|+\left|\theta\left(t^{k}\right)-\theta_{h}^{k}\right|+h\right), \\
\left\|\boldsymbol{\nabla} \mathcal{A}\left(t^{k} ; 0, \cdot\right)-\boldsymbol{\nabla} \mathcal{A}_{h}^{k}\right\|_{L^{2}(\mathcal{O})^{4}} \leqslant C\left(\left|\boldsymbol{\zeta}\left(t^{k}\right)-\boldsymbol{\zeta}_{h}^{k}\right|+\left|\theta\left(t^{k}\right)-\theta_{h}^{k}\right|+h^{1 / 2}\right) .
\end{gathered}
$$

Proof. From definitions (2.16) and (3.5), one has, for all $\boldsymbol{x}$ in $\mathcal{O}$,

$$
\mathcal{A}\left(t^{k} ; 0, \boldsymbol{x}\right)-\mathcal{A}_{h}^{k}(\boldsymbol{x})=\boldsymbol{d}\left(\boldsymbol{x}, t^{k}\right)-\boldsymbol{d}_{h}^{k}(\boldsymbol{x}) .
$$

On the one hand, error estimates (4.26) and (4.25) in [11] respectively yield

$$
\begin{gathered}
\left\|\check{\boldsymbol{d}}_{h}^{k}-\boldsymbol{d}_{h}^{k}\right\|_{\mathrm{L}^{\infty}\left(\mathcal{F}_{h}^{0}\right)^{2}} \leqslant C h^{2}|\log h|\left(\left|\boldsymbol{\zeta}_{h}^{k}\right|+\left|\theta_{h}^{k}\right|\right), \\
\left\|\boldsymbol{\nabla} \check{\boldsymbol{d}}_{h}^{k}-\boldsymbol{\nabla} \boldsymbol{d}_{h}^{k}\right\|_{L^{2}\left(\mathcal{F}_{h}^{0}\right)^{4}} \leqslant C h\left(\left|\boldsymbol{\zeta}_{h}^{k}\right|+\left|\theta_{h}^{k}\right|\right),
\end{gathered}
$$


and, on the other hand, it holds

$$
\begin{aligned}
& \left\|\boldsymbol{d}\left(\cdot, t^{k}\right)-\check{\boldsymbol{d}}_{h}^{k}\right\|_{\mathrm{L}^{\infty}(\mathcal{O})^{2}} \leqslant C\left(\left|\boldsymbol{\zeta}\left(t^{k}\right)-\boldsymbol{\zeta}_{h}^{k}\right|+\left|\theta\left(t^{k}\right)-\theta_{h}^{k}\right|\right) \\
& \left\|\boldsymbol{\nabla} \boldsymbol{d}\left(\cdot, t^{k}\right)-\nabla \check{\boldsymbol{d}}_{h}^{k}\right\|_{\mathrm{L}^{2}(\mathcal{O})^{4}} \leqslant C\left(\left|\boldsymbol{\zeta}\left(t^{k}\right)-\boldsymbol{\zeta}_{h}^{k}\right|+\left|\theta\left(t^{k}\right)-\theta_{h}^{k}\right|\right) \text {. }
\end{aligned}
$$

We infer from these relations and the triangle inequality that

$$
\begin{gathered}
\left\|\boldsymbol{d}\left(\cdot, t^{k}\right)-\boldsymbol{d}_{h}^{k}\right\|_{L^{\infty}\left(\mathcal{F}_{h}^{0}\right)^{2}} \leqslant C\left(\left|\boldsymbol{\zeta}\left(t^{k}\right)-\boldsymbol{\zeta}_{h}^{k}\right|+\left|\theta\left(t^{k}\right)-\theta_{h}^{k}\right|+h^{2}|\log h|\right), \\
\left\|\boldsymbol{\nabla} \boldsymbol{d}\left(\cdot, t^{k}\right)-\nabla \boldsymbol{d}_{h}^{k}\right\|_{L^{2}\left(\mathcal{F}_{h}^{0}\right)^{4}} \leqslant C\left(\left|\boldsymbol{\zeta}\left(t^{k}\right)-\boldsymbol{\zeta}_{h}^{k}\right|+\left|\theta\left(t^{k}\right)-\theta_{h}^{k}\right|+h\right) .
\end{gathered}
$$

Then, given the form of the extensions of the ALE mappings (see Rems. 2.4 and 3.1), it is easy to check that

$$
\begin{gathered}
\left\|\boldsymbol{d}\left(\cdot, t^{k}\right)-\boldsymbol{d}_{h}^{k}\right\|_{L^{\infty}(\mathcal{S})^{2}} \leqslant C\left(\left|\boldsymbol{\zeta}\left(t^{k}\right)-\boldsymbol{\zeta}_{h}^{k}\right|+\left|\theta\left(t^{k}\right)-\theta_{h}^{k}\right|\right), \\
\left\|\boldsymbol{\nabla} \boldsymbol{d}\left(\cdot, t^{k}\right)-\boldsymbol{\nabla} \boldsymbol{d}_{h}^{k}\right\|_{\mathrm{L}^{2}(\mathcal{S})^{4}} \leqslant C\left|\theta\left(t^{k}\right)-\theta_{h}^{k}\right| .
\end{gathered}
$$

Finally, owing to the mean value theorem and property (3.2), it follows that

$$
\left\|\boldsymbol{d}\left(\cdot, t^{k}\right)-\boldsymbol{d}_{h}^{k}\right\|_{\mathrm{L}^{\infty}\left(\mathcal{F} \backslash \mathcal{F}_{h}^{0}\right)^{2}} \leqslant C\left(h\left\|\boldsymbol{d}\left(\cdot, t^{k}\right)\right\|_{\mathrm{W}^{1, \infty}(\mathcal{F})^{2}}+\left|\boldsymbol{\zeta}\left(t^{k}\right)-\boldsymbol{\zeta}_{h}^{k}\right|+\left|\theta\left(t^{k}\right)-\theta_{h}^{k}\right|\right)
$$

and, using directly (3.2),

$$
\left\|\boldsymbol{\nabla} \boldsymbol{d}\left(\cdot, t^{k}\right)-\boldsymbol{\nabla} \boldsymbol{d}_{h}^{k}\right\|_{\mathrm{L}^{2}\left(\mathcal{F} \backslash \mathcal{F}_{h}^{0}\right)^{4}} \leqslant C h^{1 / 2}\left(\left\|\boldsymbol{d}\left(\cdot, t^{k}\right)\right\|_{\mathrm{W}^{1, \infty}(\mathcal{F})^{2}}+\left|\mathbf{R}_{\theta_{h}^{k}}-\mathbf{I d}\right|\right),
$$

which ends the proof of the lemma.

It is also natural to compare the discrete mapping $\mathcal{A}_{h}^{k}$ with the diffeomorphism

$$
\widehat{\mathcal{A}}^{k}=\mathbf{Y}^{k} \circ \mathcal{A}\left(t^{k} ; 0, \cdot\right)
$$

which verifies $\widehat{\mathcal{A}}^{k}(\mathcal{F})=\mathcal{F}\left(\boldsymbol{\zeta}_{h}^{k}, \theta_{h}^{k}\right)$. From assumptions (2.1) and Lemma 4.1, we have

$$
\left\|\widehat{\mathcal{A}}^{k}\right\|_{\mathrm{W}^{2, \infty}(\mathcal{O})^{2}} \leqslant C, 0 \leqslant k \leqslant N
$$

and, using (5.4) and Lemma 4.1, we can also see that

$$
\left\|\left(\widehat{\mathcal{A}}^{k}\right)^{-1}\right\|_{\mathrm{W}^{2, \infty}(\mathcal{O})^{2}} \leqslant C, 0 \leqslant k \leqslant N .
$$

Lemma 5.2. There exists a constant $C$ such that, for all $k \in\{0, \ldots, N\}$,

$$
\begin{gathered}
\left\|\widehat{\mathcal{A}}^{k}-\mathcal{A}_{h}^{k}\right\|_{\mathrm{L}^{\infty}(\mathcal{O})^{2}} \leqslant C\left(\left|\boldsymbol{\zeta}\left(t^{k}\right)-\boldsymbol{\zeta}_{h}^{k}\right|+\left|\theta\left(t^{k}\right)-\theta_{h}^{k}\right|+h\right), \\
\left\|\left(\widehat{\mathcal{A}}^{k}\right)^{-1}-\left(\mathcal{A}_{h}^{k}\right)^{-1}\right\|_{L^{\infty}(\mathcal{O})^{2}} \leqslant C\left(\left|\boldsymbol{\zeta}\left(t_{k}\right)-\boldsymbol{\zeta}_{h}^{k}\right|+\left|\theta\left(t_{k}\right)-\theta_{h}^{k}\right|+h\right) .
\end{gathered}
$$

Proof. The first assertion is an easy consequence of Lemmata 4.1 and 5.1. To prove the second one, we write $\left\|\left(\widehat{\mathcal{A}}^{k}\right)^{-1}-\left(\mathcal{A}_{h}^{k}\right)^{-1}\right\|_{L^{\infty}(\mathcal{O})^{2}}=\left\|\left(\widehat{\mathcal{A}}^{k}\right)^{-1} \circ \mathcal{A}_{h}^{k}-\left(\mathcal{A}_{h}^{k}\right)^{-1} \circ \mathcal{A}_{h}^{k}\right\|_{L^{\infty}(\mathcal{O})^{2}}=\left\|\left(\widehat{\mathcal{A}}^{k}\right)^{-1} \circ \mathcal{A}_{h}^{k}-\left(\widehat{\mathcal{A}}^{k}\right)^{-1} \circ \widehat{\mathcal{A}}^{k}\right\|_{L^{\infty}(\mathcal{O})^{2}}$. 
Consequently, using the mean value theorem and (5.7), we find

$$
\left\|\left(\widehat{\mathcal{A}}^{k}\right)^{-1}-\left(\mathcal{A}_{h}^{k}\right)^{-1}\right\|_{L^{\infty}(\mathcal{O})^{2}} \leqslant C\left\|\widehat{\mathcal{A}}^{k}-\mathcal{A}_{h}^{k}\right\|_{L^{\infty}(\mathcal{O})^{2}} .
$$

The above inequality and the first claim of the lemma then imply the desired result.

We next recall a classical result (see [1]).

Lemma 5.3. Assume that $\boldsymbol{Z}_{i}: \mathcal{O} \rightarrow \mathcal{O}(i=1,2)$ are two diffeomorphisms such that, for all $\mu$ in $[0,1]$,

$$
\mu \boldsymbol{Z}_{1}+(1-\mu) \boldsymbol{Z}_{2}: \mathcal{O} \rightarrow \mathcal{O}
$$

is a diffeomorphism with Jacobian $J_{\mu}$ satisfying

$$
J_{\mu} \geqslant c
$$

with $c$ a positive constant. Then, for any $\gamma$ in $\mathrm{H}^{1}(\mathcal{O})$, we have

$$
\left\|\gamma \circ \boldsymbol{Z}_{1}-\gamma \circ \boldsymbol{Z}_{2}\right\|_{\mathrm{L}^{1}(\mathcal{O})} \leqslant \frac{1}{c}\|\boldsymbol{\nabla} \gamma\|_{\mathrm{L}^{2}(\mathcal{O})^{2}}\left\|\boldsymbol{Z}_{1}-\boldsymbol{Z}_{2}\right\|_{\mathrm{L}^{2}(\mathcal{O})^{2}} .
$$

From the above lemma, we infer the following.

Lemma 5.4. Assume that $\delta t$ and $h$ are both small enough. Then, for all $k$ in $\{0, \ldots, N\}$ and $\mu$ in $[0,1]$, the mapping

$$
\mu \widehat{\mathcal{A}}^{k}+(1-\mu) \mathcal{A}_{h}^{k}: \mathcal{O} \rightarrow \mathcal{O}
$$

is a diffeomorphism with Jacobian satisfying inequality (5.8). The same property holds for the family of mappings

$$
\mu\left(\widehat{\mathcal{A}}^{k}\right)^{-1}+(1-\mu)\left(\mathcal{A}_{h}^{k}\right)^{-1}: \mathcal{O} \rightarrow \mathcal{O} .
$$

Proof. Recall that the constant $c_{0}$ in (2.17) has been chosen in such a way that bound (2.18) is satisfied. We first deduce from estimate (3.9) and hypothesis (4.1) that, for all $k$ in $\{0, \ldots, N\}$,

$$
\left\|\boldsymbol{d}_{h}^{k}\right\|_{\mathrm{W}^{1, \infty}(\mathcal{O})^{2}} \leqslant C\left(c_{0}+\delta t+h^{1 / 2}\right) .
$$

Then, choosing $c_{0}, \bar{h}$ and $\kappa$ small enough, we obtain that, for all $h$ in $(0, \bar{h})$ and $\delta t$ in $(0, \kappa)$,

$$
\left\|\boldsymbol{d}_{h}^{k}\right\|_{\mathrm{W}^{1, \infty}(\mathcal{O})^{2}} \leqslant \frac{1}{4}
$$

For all $k$ in $\{0, \ldots, N\}$ and $\mu$ in $[0,1]$, this implies that

$$
\left\|\mu \boldsymbol{d}\left(\cdot, t^{k}\right)+(1-\mu) \boldsymbol{d}_{h}^{k}\right\|_{\mathrm{W}^{1, \infty}(\mathcal{O})^{2}} \leqslant \frac{1}{4},
$$

which yields the first result (see the proof of Lem. 2.2 for details). Next, using again bounds (2.18) and (5.9), we infer that, for all $k$ in $\{0, \ldots, N\}$,

$$
\left(\widehat{\mathcal{A}}^{k}\right)^{-1}=\mathbf{I d}+\underline{\boldsymbol{d}}^{k},\left(\mathcal{A}_{h}^{k}\right)^{-1}=\mathbf{I d}+\underline{\boldsymbol{d}}_{h}^{k}
$$

with

$$
\left\|\underline{\boldsymbol{d}}^{k}\right\|_{\mathrm{W}^{1, \infty}(\mathcal{O})^{2}} \leqslant \frac{1}{2} \text { and }\left\|\underline{\boldsymbol{d}}_{h}^{k}\right\|_{\mathrm{W}^{1, \infty}(\mathcal{O})^{2}} \leqslant \frac{1}{2}
$$

so that, for all $\mu$ in $[0,1]$, the mapping $\mu\left(\widehat{\mathcal{A}}^{k}\right)^{-1}+(1-\mu)\left(\mathcal{A}_{h}^{k}\right)^{-1}$ is a diffeomorphism of $\mathcal{O}$ onto itself with Jacobian satisfying (5.8). 
We are now in position to prove the following result.

Lemma 5.5. There exists a constant $C$ such that, for all $k \in\{0, \ldots, N-1\}$,

$$
\left\|\left[\boldsymbol{\nabla}\left(\widehat{\mathcal{A}}^{k} \circ\left(\widehat{\mathcal{A}}^{k+1}\right)^{-1}\right)\right]^{-1}-\left[\boldsymbol{\nabla} \mathcal{A}_{h}^{k+1, k}\right]^{-1}\right\|_{L^{2}(\mathcal{O})^{4}} \leqslant C\left(\left|\boldsymbol{\zeta}\left(t^{k}\right)-\boldsymbol{\zeta}_{h}^{k}\right|+\left|\theta\left(t^{k}\right)-\theta_{h}^{k}\right|+h^{1 / 2}\right) .
$$

Proof. Differentiating, we have respectively

$$
\left[\nabla\left(\widehat{\mathcal{A}}^{k} \circ\left(\hat{\mathcal{A}}^{k+1}\right)^{-1}\right)\right]^{-1}=\left[\nabla \widehat{\mathcal{A}}^{k+1}\left(\left(\widehat{\mathcal{A}}^{k+1}\right)^{-1}\right)\right]\left[\nabla \widehat{\mathcal{A}}^{k}\left(\left(\widehat{\mathcal{A}}^{k+1}\right)^{-1}\right)\right]^{-1}
$$

and

$$
\left[\boldsymbol{\nabla} \mathcal{A}_{h}^{k+1, k}\right]^{-1}=\left[\boldsymbol{\nabla} \mathcal{A}_{h}^{k+1}\left(\left(\mathcal{A}_{h}^{k+1}\right)^{-1}\right)\right]\left[\nabla \mathcal{A}_{h}^{k}\left(\left(\mathcal{A}_{h}^{k+1}\right)^{-1}\right)\right]^{-1} .
$$

We use this to rewrite the following difference as the sum of four terms:

$$
\left[\boldsymbol{\nabla}\left(\widehat{\mathcal{A}}^{k} \circ\left(\widehat{\mathcal{A}}^{k+1}\right)^{-1}\right)\right]^{-1}-\left[\nabla \mathcal{A}_{h}^{k+1, k}\right]^{-1}=\sum_{i=1}^{4} R_{i}
$$

where

$$
\begin{aligned}
& R_{1}=\left[\nabla \widehat{\mathcal{A}}^{k+1}\left(\left(\widehat{\mathcal{A}}^{k+1}\right)^{-1}\right)\right]\left[\nabla \widehat{\mathcal{A}}^{k}\left(\left(\widehat{\mathcal{A}}^{k+1}\right)^{-1}\right)\right]^{-1}-\left[\boldsymbol{\nabla} \widehat{\mathcal{A}}^{k+1}\left(\left(\mathcal{A}_{h}^{k+1}\right)^{-1}\right)\right]\left[\nabla \widehat{\mathcal{A}}^{k}\left(\left(\widehat{\mathcal{A}}^{k+1}\right)^{-1}\right)\right]^{-1}, \\
& R_{2}=\left[\nabla \widehat{\mathcal{A}}^{k+1}\left(\left(\mathcal{A}_{h}^{k+1}\right)^{-1}\right)\right]\left[\nabla \widehat{\mathcal{A}}^{k}\left(\left(\hat{\mathcal{A}}^{k+1}\right)^{-1}\right)\right]^{-1}-\left[\boldsymbol{\nabla} \mathcal{A}_{h}^{k+1}\left(\left(\mathcal{A}_{h}^{k+1}\right)^{-1}\right)\right]\left[\nabla \widehat{\mathcal{A}}^{k}\left(\left(\hat{\mathcal{A}}^{k+1}\right)^{-1}\right)\right]^{-1}, \\
& R_{3}=\left[\nabla \mathcal{A}_{h}^{k+1}\left(\left(\mathcal{A}_{h}^{k+1}\right)^{-1}\right)\right]\left[\left[\nabla \widehat{\mathcal{A}}^{k}\left(\left(\widehat{\mathcal{A}}^{k+1}\right)^{-1}\right)\right]^{-1}-\left[\nabla \widehat{\mathcal{A}}^{k}\left(\left(\mathcal{A}_{h}^{k+1}\right)^{-1}\right)\right]^{-1}\right] \\
& R_{4}=\left[\boldsymbol{\nabla} \mathcal{A}_{h}^{k+1}\left(\left(\mathcal{A}_{h}^{k+1}\right)^{-1}\right)\right]\left[\nabla \widehat{\mathcal{A}}^{k}\left(\left(\mathcal{A}_{h}^{k+1}\right)^{-1}\right)\right]^{-1}-\left[\boldsymbol{\nabla} \mathcal{A}_{h}^{k+1}\left(\left(\mathcal{A}_{h}^{k+1}\right)^{-1}\right)\right]\left[\nabla \mathcal{A}_{h}^{k}\left(\left(\mathcal{A}_{h}^{k+1}\right)^{-1}\right)\right]^{-1} .
\end{aligned}
$$

Using bounds (5.6) and (5.7), we infer from Lemma 5.4 that

$$
\left\|R_{1}\right\|_{\mathrm{L}^{2}(\mathcal{O})^{4}} \leqslant C\left\|\left(\widehat{\mathcal{A}}^{k+1}\right)^{-1}-\left(\mathcal{A}_{h}^{k+1}\right)^{-1}\right\|_{\mathrm{L}^{2}(\mathcal{O})^{2}} .
$$

Combining the above inequality with Lemma 5.2 then yields

$$
\left\|R_{1}\right\|_{L^{2}(\mathcal{O})^{4}} \leqslant C\left(\left|\boldsymbol{\zeta}\left(t_{k}\right)-\boldsymbol{\zeta}_{h}^{k}\right|+\left|\theta\left(t_{k}\right)-\theta_{h}^{k}\right|+h\right)
$$

Next, using Lemmata 5.1 and 5.4, we obtain

$$
\left\|R_{2}\right\|_{L^{2}(\mathcal{O})^{4}} \leqslant C\left(\left|\boldsymbol{\zeta}\left(t_{k}\right)-\zeta_{h}^{k}\right|+\left|\theta\left(t_{k}\right)-\theta_{h}^{k}\right|+h^{1 / 2}\right) .
$$

To estimate $R_{3}$, we first write

$$
\begin{aligned}
{\left[\nabla \widehat{\mathcal{A}}^{k}\left(\left(\widehat{\mathcal{A}}^{k+1}\right)^{-1}\right)\right]^{-1}-\left[\nabla \widehat{\mathcal{A}}^{k}\left(\left(\mathcal{A}_{h}^{k+1}\right)^{-1}\right)\right]^{-1}=} & \\
& {\left[\nabla \widehat{\mathcal{A}}^{k}\left(\left(\widehat{\mathcal{A}}^{k+1}\right)^{-1}\right)\right]^{-1}\left[\nabla \widehat{\mathcal{A}}^{k}\left(\left(\mathcal{A}_{h}^{k+1}\right)^{-1}\right)-\nabla \widehat{\mathcal{A}}^{k}\left(\left(\widehat{\mathcal{A}}^{k+1}\right)^{-1}\right)\right]\left[\nabla \widehat{\mathcal{A}}^{k}\left(\left(\mathcal{A}_{h}^{k+1}\right)^{-1}\right)\right]^{-1} }
\end{aligned}
$$


Consequently, using Lemmata 5.2, 5.3 and 5.4, we get

$$
\left\|R_{3}\right\|_{\mathrm{L}^{2}(\mathcal{O})^{4}} \leqslant C\left\|\left(\widehat{\mathcal{A}}^{k+1}\right)^{-1}-\left(\mathcal{A}_{h}^{k+1}\right)^{-1}\right\|_{L^{2}(\mathcal{O})^{2}} \leqslant C\left(\left|\boldsymbol{\zeta}\left(t_{k}\right)-\boldsymbol{\zeta}_{h}^{k}\right|+\left|\theta\left(t_{k}\right)-\theta_{h}^{k}\right|+h\right) .
$$

Finally, using the relation

$$
\left[\boldsymbol{\nabla} \widehat{\mathcal{A}}^{k}\right]^{-1}-\left[\boldsymbol{\nabla} \mathcal{A}_{h}^{k}\right]^{-1}=\left[\boldsymbol{\nabla} \widehat{\mathcal{A}}^{k}\right]^{-1}\left(\boldsymbol{\nabla} \mathcal{A}_{h}^{k}-\boldsymbol{\nabla} \widehat{\mathcal{A}}^{k}\right)\left[\boldsymbol{\nabla} \mathcal{A}_{h}^{k}\right]^{-1}
$$

in conjunction with Lemmata 5.1, 5.3 and 5.4, it can be shown that

$$
\left\|R_{4}\right\|_{\mathrm{L}^{2}(\mathcal{O})^{4}} \leqslant C\left(\left|\boldsymbol{\zeta}\left(t_{k}\right)-\boldsymbol{\zeta}_{h}^{k}\right|+\left|\theta\left(t_{k}\right)-\theta_{h}^{k}\right|+h^{1 / 2}\right),
$$

which completes the proof.

We now derive an error estimate for the discrete domain velocity. Denote

$$
\mathbf{W}^{k}(\boldsymbol{y})=J_{\mathbf{Y}^{k}}\left(\mathbf{X}^{k}(\boldsymbol{y})\right) \boldsymbol{w}\left(\mathbf{X}^{k}(\boldsymbol{y}), t^{k}\right)
$$

We have the following result.

Lemma 5.6. There exists a constant $C$ such that, for all $k \in\{0, \ldots, N\}$,

$$
\left\|\mathbf{W}^{k}-\boldsymbol{w}_{h}^{k}\right\|_{\mathrm{L}^{2}(\mathcal{O})^{2}} \leqslant C\left(\left|\boldsymbol{\zeta}\left(t^{k}\right)-\boldsymbol{\zeta}_{h}^{k}\right|+\left|\theta\left(t^{k}\right)-\theta_{h}^{k}\right|+\left|\boldsymbol{\xi}\left(t^{k}\right)-\boldsymbol{\xi}_{h}^{k}\right|+\left|\omega\left(t^{k}\right)-\omega_{h}^{k}\right|+\delta t+h\right) .
$$

Proof. From definition (2.9) of the domain velocity and identity (2.16) defining the ALE mapping, it follows that

$$
\boldsymbol{w}\left(\mathcal{A}\left(t^{k+1} ; 0, \cdot\right), t\right)=\frac{\partial \mathcal{A}}{\partial t}(t ; 0, \cdot)=\frac{\partial \boldsymbol{d}}{\partial t}(\cdot, t) \text { in } \mathcal{F}
$$

where $\frac{\partial \boldsymbol{d}}{\partial t}(\cdot, t)$ is solution to problem (5.1). On the other hand, combining definitions (3.11), (3.12) and (3.13), we obtain that

$$
\boldsymbol{w}_{h}^{k} \circ \mathcal{A}_{h}^{k}=\frac{\mathcal{A}_{h}^{k+1}-\mathcal{A}_{h}^{k}}{\delta t}
$$

Moreover, using (3.5), (3.6) and (3.7), an easy computation shows that the field $\Upsilon_{h}^{k}=\frac{\mathcal{A}_{h}^{k+1}-\mathcal{A}_{h}^{k}}{\delta t}$ satisfies

$$
\boldsymbol{\Upsilon}_{h}^{k}(\boldsymbol{x})=\boldsymbol{\xi}_{h}^{k+1}+\omega_{h}^{k} \mathbf{R}_{\theta_{h}^{k}} \boldsymbol{x}^{\perp}+(\delta t) \boldsymbol{e}_{\Upsilon_{h}^{k}}(\boldsymbol{x}), \forall \boldsymbol{x} \in \partial \mathcal{S}_{h}^{0}, \text { and } \boldsymbol{\Upsilon}_{h}^{k}=\mathbf{0} \text { on } \partial \mathcal{O}
$$

where

$$
\boldsymbol{e}_{\Upsilon_{h}^{k}}(\boldsymbol{x})=-\left(\omega_{h}^{k}\right)^{2} \int_{0}^{1}(1-s) \mathbf{R}_{s(\delta t) \omega_{h}^{k}} \boldsymbol{x} \mathrm{d} s
$$

and

$$
\int_{\mathcal{F}_{h}^{0}} \boldsymbol{\nabla} \boldsymbol{\Upsilon}_{h}^{k}: \nabla \gamma_{h} \mathrm{~d} \boldsymbol{x}+\lambda \int_{\mathcal{F}_{h}^{0}}\left(\operatorname{div} \boldsymbol{\Upsilon}_{h}^{k}\right)\left(\operatorname{div} \boldsymbol{\gamma}_{h}\right) \mathrm{d} \boldsymbol{x}=0, \forall \boldsymbol{\gamma}_{h} \in\left(\mathcal{P}_{h}^{0}\right)^{2} \text { s.t. } \boldsymbol{\gamma}_{h}=\mathbf{0} \text { on } \partial \mathcal{F}_{h}^{0}
$$

Then, using inequality (4.26) in reference [11] and proceeding as in the proof of Lemma 5.1, we deduce that

$$
\left\|\frac{\partial \mathcal{A}}{\partial t}\left(t^{k} ; 0, \cdot\right)-\frac{\mathcal{A}_{h}^{k+1}-\mathcal{A}_{h}^{k}}{\delta t}\right\|_{L^{2}(\mathcal{O})^{2}} \leqslant C\left(\left|\boldsymbol{\xi}\left(t^{k}\right)-\boldsymbol{\xi}_{h}^{k}\right|+\left|\omega\left(t^{k}\right)-\omega_{h}^{k}\right|+\delta t+h\right) .
$$


According to the definition of $\mathbf{W}^{k}$, we can write

$$
\begin{aligned}
\mathbf{W}^{k}-\boldsymbol{w}_{h}^{k}=J_{\mathbf{Y}^{k}}\left(\mathbf{X}^{k}\right) \boldsymbol{w}\left(\mathbf{X}^{k}, t^{k}\right)-\boldsymbol{w}_{h}^{k}=\left(J_{\mathbf{Y}^{k}}\left(\mathbf{X}^{k}\right)-\mathbf{I d}\right) \boldsymbol{w}\left(\mathbf{X}^{k}, t^{k}\right) & +\frac{\partial \boldsymbol{d}}{\partial t}\left(\left(\widehat{\mathcal{A}}^{k}\right)^{-1}, t^{k}\right)-\frac{\partial \boldsymbol{d}}{\partial t}\left(\left(\mathcal{A}_{h}^{k}\right)^{-1}, t^{k}\right) \\
& +\frac{\partial \boldsymbol{d}}{\partial t}\left(\left(\mathcal{A}_{h}^{k}\right)^{-1}, t^{k}\right)-\frac{\mathcal{A}_{h}^{k+1}-\mathcal{A}_{h}^{k}}{\delta t} \circ\left(\mathcal{A}_{h}^{k}\right)^{-1} .
\end{aligned}
$$

Assumptions (2.1) and Lemma 4.1 then give

$$
\left\|\left(J_{\mathbf{Y}^{k}}\left(\mathbf{X}^{k}\right)-\mathbf{I d}\right) \boldsymbol{w}\left(\mathbf{X}^{k}, t^{k}\right)\right\|_{L^{2}(\mathcal{O})^{2}} \leqslant C\left(\left|\boldsymbol{\zeta}\left(t^{k}\right)-\boldsymbol{\zeta}_{h}^{k}\right|+\left|\theta\left(t^{k}\right)-\theta_{h}^{k}\right|\right) .
$$

From the regularity of $\boldsymbol{d}$ and Lemma 5.2, we deduce that

$$
\left\|\frac{\partial \boldsymbol{d}}{\partial t}\left(\left(\widehat{\mathcal{A}}^{k}\right)^{-1}, t^{k}\right)-\frac{\partial \boldsymbol{d}}{\partial t}\left(\left(\mathcal{A}_{h}^{k}\right)^{-1}, t^{k}\right)\right\|_{L^{2}(\mathcal{O})^{2}} \leqslant C\left(\left|\boldsymbol{\zeta}\left(t^{k}\right)-\boldsymbol{\zeta}_{h}^{k}\right|+\left|\theta\left(t^{k}\right)-\theta_{h}^{k}\right|+h\right),
$$

and, from inequality (5.11) and Lemma 5.4, we obtain

$$
\left\|\frac{\partial \boldsymbol{d}}{\partial t}\left(\left(\mathcal{A}_{h}^{k}\right)^{-1}, t^{k}\right)-\frac{\mathcal{A}_{h}^{k+1}-\mathcal{A}_{h}^{k}}{\delta t} \circ\left(\mathcal{A}_{h}^{k}\right)^{-1}\right\|_{L^{2}(\mathcal{O})^{2}} \leqslant C\left(\left|\boldsymbol{\xi}\left(t^{k}\right)-\boldsymbol{\xi}_{h}^{k}\right|+\left|\omega\left(t^{k}\right)-\omega_{h}^{k}\right|+\delta t+h\right) .
$$

The last four relations and the triangle inequality establish the result.

Lemma 5.7. There exists a constant $C$ such that, for all $k \in\{0, \ldots, N-1\}$,

$$
\begin{aligned}
&\left\|\widehat{\mathcal{A}}^{k} \circ\left(\widehat{\mathcal{A}}^{k+1}\right)^{-1}-\mathcal{A}_{h}^{k} \circ\left(\mathcal{A}_{h}^{k+1}\right)^{-1}\right\|_{L^{2}(\mathcal{O})^{2}} \leqslant \\
& C(\delta t)\left(\left|\boldsymbol{\zeta}\left(t^{k}\right)-\boldsymbol{\zeta}_{h}^{k}\right|+\left|\theta\left(t^{k}\right)-\theta_{h}^{k}\right|+\left|\boldsymbol{\xi}\left(t^{k}\right)-\boldsymbol{\xi}_{h}^{k}\right|+\left|\omega\left(t^{k}\right)-\omega_{h}^{k}\right|+\delta t+h\right) .
\end{aligned}
$$

Proof. We have

$$
\begin{aligned}
\widehat{\mathcal{A}}^{k} \circ\left(\widehat{\mathcal{A}}^{k+1}\right)^{-1}-\mathcal{A}_{h}^{k} \circ\left(\mathcal{A}_{h}^{k+1}\right)^{-1}= & \left(\widehat{\mathcal{A}}^{k}-\widehat{\mathcal{A}}^{k+1}\right) \circ\left(\widehat{\mathcal{A}}^{k+1}\right)^{-1}-\left(\mathcal{A}_{h}^{k}-\mathcal{A}_{h}^{k+1}\right) \circ\left(\mathcal{A}_{h}^{k+1}\right)^{-1} \\
= & \left(\mathcal{A}_{h}^{k+1}-\mathcal{A}_{h}^{k}\right) \circ\left(\mathcal{A}_{h}^{k+1}\right)^{-1}-\left(\mathcal{A}^{k}-\mathcal{A}^{k+1}\right) \circ\left(\widehat{\mathcal{A}}^{k+1}\right)^{-1} \\
& +\left(\mathbf{Y}^{k}-\mathbf{Y}^{k+1}\right) \circ \mathcal{A}^{k} \circ\left(\widehat{\mathcal{A}}^{k+1}\right)^{-1} \\
& +\left(\mathbf{Y}^{k}-\mathbf{I d}\right) \circ\left(\mathcal{A}^{k}-\mathcal{A}^{k+1}\right) \circ\left(\widehat{\mathcal{A}}^{k+1}\right)^{-1} .
\end{aligned}
$$

Owing to (3.18), the mapping $\mathcal{A}(\cdot ; 0, \cdot)$ belongs to $\mathrm{W}^{2, \infty}\left(0, T ; \mathrm{L}^{2}(\mathcal{O})^{2}\right)$ and we thus can write

$$
\frac{\mathcal{A}^{k}-\mathcal{A}^{k+1}}{\delta t} \circ\left(\widehat{\mathcal{A}}^{k+1}\right)^{-1}=\frac{\partial \mathcal{A}}{\partial t}\left(t^{k} ; 0, \cdot\right) \circ\left(\widehat{\mathcal{A}}^{k+1}\right)^{-1}+\boldsymbol{e}_{\partial_{t}}^{k} \mathcal{A}
$$

with

$$
\left\|\boldsymbol{e}_{\partial_{t} \mathcal{A}}^{k}\right\|_{L^{2}(\mathcal{O})^{2}} \leqslant C(\delta t)
$$


Consequently, using (5.2), (5.6) and Lemma 5.4, we deduce that

$$
\begin{aligned}
\left\|\hat{\mathcal{A}}^{k} \circ\left(\widehat{\mathcal{A}}^{k+1}\right)^{-1}-\mathcal{A}_{h}^{k} \circ\left(\mathcal{A}_{h}^{k+1}\right)^{-1}\right\|_{L^{2}(\mathcal{O})^{2}} \leqslant C(\delta t) & \left(\left\|\frac{\partial \mathcal{A}}{\partial t}\left(t^{k} ; 0, \cdot\right)-\frac{\mathcal{A}_{h}^{k+1}-\mathcal{A}_{h}^{k}}{\delta t}\right\|_{L^{2}(\mathcal{O})^{2}}\right. \\
& \left.+\left\|\left(\widehat{\mathcal{A}}^{k+1}\right)^{-1}-\left(\mathcal{A}_{h}^{k+1}\right)^{-1}\right\|_{L^{2}(\mathcal{O})^{2}}+\left\|\frac{\mathbf{Y}^{k}-\mathbf{Y}^{k+1}}{\delta t}\right\|_{L^{2}(\mathcal{O})^{2}}+\left\|\mathbf{Y}^{k}-\mathbf{I d}\right\|_{L^{2}(\mathcal{O})^{2}}+\delta t\right) .
\end{aligned}
$$

Combining the above inequality with estimate (5.11) and Lemmata 4.1, 4.3 and 5.2 then gives the result.

The following lemma is stated without proof, since it follows from arguments similar to those used to show Lemmata 5.1 and 5.2 (using regularity properties (5.2) and (5.7)).

Lemma 5.8. There exists a constant $C$ such that, for all $k \in\{0, \ldots, N\}$,

$$
\begin{gathered}
\left\|\mathcal{A}\left(t^{k} ; 0, \cdot\right)-\mathcal{A}_{h}^{k}\right\|_{\mathrm{W}^{1, \infty}(\mathcal{O})^{2}} \leqslant C\left(\left|\boldsymbol{\zeta}\left(t^{k}\right)-\boldsymbol{\zeta}_{h}^{k}\right|+\left|\theta\left(t^{k}\right)-\theta_{h}^{k}\right|+h|\log h|\right), \\
\left\|\left(\widehat{\mathcal{A}}^{k}\right)^{-1}-\left(\mathcal{A}_{h}^{k}\right)^{-1}\right\|_{\mathrm{W}^{1, \infty}(\mathcal{O})^{2}} \leqslant C\left(\left|\boldsymbol{\zeta}\left(t_{k}\right)-\boldsymbol{\zeta}_{h}^{k}\right|+\left|\theta\left(t_{k}\right)-\theta_{h}^{k}\right|+h|\log h|\right) .
\end{gathered}
$$

As a corollary, we have:

Corollary 5.9. There exists a constant $C$ such that, for all $k \in\{0, \ldots, N\}$,

$$
\begin{gathered}
\left\|\mathcal{A}_{h}^{k}\right\|_{\mathrm{W}^{1, \infty}(\mathcal{O})^{2}} \leqslant C\left(1+\left|\boldsymbol{\zeta}\left(t^{k}\right)-\boldsymbol{\zeta}_{h}^{k}\right|+\left|\theta\left(t^{k}\right)-\theta_{h}^{k}\right|\right), \\
\left\|\left(\mathcal{A}_{h}^{k}\right)^{-1}\right\|_{\mathrm{W}^{1, \infty}(\mathcal{O})^{2}} \leqslant C\left(1+\left|\boldsymbol{\zeta}\left(t_{k}\right)-\boldsymbol{\zeta}_{h}^{k}\right|+\left|\theta\left(t_{k}\right)-\theta_{h}^{k}\right|\right) .
\end{gathered}
$$

In particular, if we denote by $h^{k}$ the discretization parameter relative to $\widetilde{\mathscr{T}_{h}^{k}}$, then there exist two positive constants $c_{1}$ and $c_{2}$ such that, for all $k \in\{0, \ldots, N\}$,

$$
c_{1} h\left(1+\left|\boldsymbol{\zeta}\left(t_{k}\right)-\boldsymbol{\zeta}_{h}^{k}\right|+\left|\theta\left(t_{k}\right)-\theta_{h}^{k}\right|\right) \leqslant h^{k} \leqslant c_{2} h\left(1+\left|\boldsymbol{\zeta}\left(t_{k}\right)-\boldsymbol{\zeta}_{h}^{k}\right|+\left|\theta\left(t_{k}\right)-\theta_{h}^{k}\right|\right) .
$$

\section{ERROR BOUNDS ON THE CHARACTERISTICS}

In the forthcoming proof of the main result, we will need some estimates on the difference $\mathcal{C}^{k}-\mathcal{A}_{h}^{k+1, k} \circ \mathcal{B}_{h}^{k}$ between the exact and approximated characteristics. We assume that (4.1) holds and that $\delta t$ and $h$ are small enough, so that all the previously established results are valid. We still suppose that $\delta t$ and $h$ are smaller than unity to simplify the estimates.

Let us recall once more that the various mappings are now defined in $\mathbb{R}^{2}$. In the sequel, the diffeomorphisms $\mathbf{X}^{k}$ and $\mathbf{Y}^{k}$ are extended to $\mathbb{R}^{2}$ by setting $\mathbf{X}^{k}=\mathbf{Y}^{k}=\mathbf{I d}$ in $\mathbb{R}^{2} \backslash \mathcal{O}$. We moreover assume that there exists a constant $\varepsilon>0$ such that the relations

$$
(\delta t)\left\|\mathbf{Y}^{k+1} \circ(\overline{\boldsymbol{u}}-\overline{\boldsymbol{w}}) \circ \mathbf{X}^{k+1}\right\|_{L^{\infty}\left(t^{k}, t^{k+1} ; \mathrm{W}^{1, \infty}(\mathcal{O})^{2}\right)} \leqslant \varepsilon
$$

and

$$
(\delta t)\left\|\overline{\boldsymbol{u}}_{\boldsymbol{h}}^{\boldsymbol{k}}-\overline{\boldsymbol{w}}_{\boldsymbol{h}}^{\boldsymbol{k}}\right\|_{\mathrm{W}^{1, \infty}(\mathcal{O})^{2}} \leqslant \varepsilon,
$$

hold, where, for all $t$ in $\left[t^{k}, t^{k+1}\right]$ and $\boldsymbol{x}$ in $\mathcal{F}\left(\boldsymbol{\zeta}\left(t^{k+1}\right), \theta\left(t^{k+1}\right)\right)$, the functions $\overline{\boldsymbol{u}}$ and $\overline{\boldsymbol{w}}$ are given by

$$
\overline{\boldsymbol{u}}(\boldsymbol{x}, t)=\left[\boldsymbol{\nabla} \mathcal{A}\left(t ; t^{k+1}, \boldsymbol{x}\right)\right]^{-1} \boldsymbol{u}\left(\mathcal{A}\left(t ; t^{k+1}, \boldsymbol{x}\right), t\right), \quad \overline{\boldsymbol{w}}(\boldsymbol{x}, t)=\left[\boldsymbol{\nabla} \mathcal{A}\left(t ; t^{k+1}, \boldsymbol{x}\right)\right]^{-1} \boldsymbol{w}\left(\mathcal{A}\left(t ; t^{k+1}, \boldsymbol{x}\right), t\right) .
$$


These assumptions are stability results to be proven by induction in Section 7 , the positive constant $\varepsilon$ being fixed below. The main result of this section is the:

Theorem 6.1. For all $k$ in $\{0, \ldots, N-1\}$, we have the following error estimate for the characteristics

$$
\left\|\mathcal{C}^{k}-\mathcal{A}_{h}^{k+1, k} \circ \mathcal{B}_{h}^{k}\right\|_{L^{2}(\mathcal{O})^{2}} \leqslant C(\delta t)\left(\left\|\mathbf{U}^{k}-\boldsymbol{u}_{h}^{k}\right\|_{L^{2}(\mathcal{O})^{2}}+\left|\boldsymbol{\zeta}\left(t^{k}\right)-\boldsymbol{\zeta}_{h}^{k}\right|+\left|\theta\left(t^{k}\right)-\theta_{h}^{k}\right|+\delta t+h^{1 / 2}\right)
$$

Auxiliary results are needed to prove Theorem 6.1. First, let us define the mapping

$$
\widehat{\mathcal{B}}\left(t ; t^{k+1}, \cdot\right)=\mathbf{Y}^{k+1} \circ \mathcal{B}\left(t ; t^{k+1}, \cdot\right) \circ \mathbf{X}^{k+1}, \forall t \in\left[t^{k}, t^{k+1}\right]
$$

and denote

$$
\widehat{\mathcal{B}}^{k}=\widehat{\mathcal{B}}\left(t^{k} ; t^{k+1}, \cdot\right)
$$

From problem $(2.20)$, we know that $\widehat{\mathcal{B}}\left(t ; t^{k+1}, \cdot\right)$ satisfies, for any $\boldsymbol{x}$ in $\mathcal{F}\left(\boldsymbol{\zeta}_{h}^{k+1}, \theta_{h}^{k+1}\right)$, the initial value problem

$$
\left\{\begin{array}{l}
\frac{\partial \widehat{\mathcal{B}}}{\partial t}\left(t ; t^{k+1}, \boldsymbol{x}\right)=\mathbf{Y}^{k+1} \circ(\overline{\boldsymbol{u}}-\overline{\boldsymbol{w}})(\cdot, t) \circ \mathbf{X}^{k+1}\left(\widehat{\mathcal{B}}\left(t ; t^{k+1}, \boldsymbol{x}\right), t\right), \\
\widehat{\mathcal{B}}\left(t^{k+1} ; t^{k+1}, \boldsymbol{x}\right)=\boldsymbol{x}
\end{array}\right.
$$

in which the functions $\overline{\boldsymbol{u}}$ and $\overline{\boldsymbol{w}}$ are defined by (6.3). Using bounds (6.1) and (6.2) for $\varepsilon$ small enough, we can show that, for any $\mu$ in $[0,1]$, the mapping $\mu \widehat{\mathcal{B}}^{k}+(1-\mu) \boldsymbol{B}_{h}^{k}$ is a diffeomorphism with Jacobian satisfying (5.8) for some positive constant $c$.

Lemma 6.2. Consider the functions $\overline{\boldsymbol{u}}$ and $\overline{\boldsymbol{w}}$ given by (6.3), and $\overline{\boldsymbol{u}}_{\boldsymbol{h}}^{\boldsymbol{k}}$ and $\overline{\boldsymbol{w}}_{\boldsymbol{h}}^{\boldsymbol{k}}$ given by (3.15). Then, for all $k$ in $\{0, \ldots, N-1\}$, we have

$$
\begin{aligned}
\int_{t^{k}}^{t^{k+1}}\left\|\mathbf{Y}^{k+1} \circ(\overline{\boldsymbol{u}}-\overline{\boldsymbol{w}})(\cdot, t) \circ \mathbf{X}^{k+1}-\left(\overline{\boldsymbol{u}}_{\boldsymbol{h}}^{\boldsymbol{k}}-\overline{\boldsymbol{w}}_{\boldsymbol{h}}^{\boldsymbol{k}}\right)\right\|_{\mathrm{L}^{2}(\mathcal{O})^{2}} \mathrm{~d} t \leqslant \\
C(\delta t)\left(\left\|\mathbf{U}^{k}-\boldsymbol{u}_{h}^{k}\right\|_{L^{2}(\mathcal{O})^{2}}+\left|\boldsymbol{\zeta}\left(t^{k}\right)-\boldsymbol{\zeta}_{h}^{k}\right|+\left|\theta\left(t^{k}\right)-\theta_{h}^{k}\right|+\delta t+h^{1 / 2}\right) .
\end{aligned}
$$

Proof. From definitions (3.15) and (6.3), it follows that, for any $\boldsymbol{y}$ in $\mathcal{O}$ and $t$ in $\left[t^{k}, t^{k+1}\right]$,

$$
\begin{aligned}
\mathbf{Y}^{k+1} \circ(\overline{\boldsymbol{u}}-\overline{\boldsymbol{w}})(\cdot, t) \circ \mathbf{X}^{k+1}(\boldsymbol{y})-\left(\overline{\boldsymbol{u}}_{\boldsymbol{h}}^{\boldsymbol{k}}-\overline{\boldsymbol{w}}_{\boldsymbol{h}}^{\boldsymbol{k}}\right)(\boldsymbol{y})= & \\
\mathbf{Y}^{k+1}\left(\left[\boldsymbol{\nabla} \mathcal{A}\left(t ; t^{k+1}, \mathbf{X}^{k+1}(\boldsymbol{y})\right)\right]^{-1}(\boldsymbol{u}-\boldsymbol{w})\left(\mathcal{A}\left(t ; t^{k+1}, \mathbf{X}^{k+1}(\boldsymbol{y})\right), t\right)\right) & -\left[\boldsymbol{\nabla} \mathcal{A}_{h}^{k+1, k}(\boldsymbol{y})\right]^{-1}\left(\boldsymbol{u}_{h}^{k}-\boldsymbol{w}_{h}^{k}\right)\left(\mathcal{A}_{h}^{k+1, k}(\boldsymbol{y})\right) .
\end{aligned}
$$

The right hand side of the above identity is then decomposed into a sum of five terms:

$$
\mathbf{Y}^{k+1} \circ(\overline{\boldsymbol{u}}-\overline{\boldsymbol{w}})(\cdot, t) \circ \mathbf{X}^{k+1}(\boldsymbol{y})-\left(\overline{\boldsymbol{u}}_{\boldsymbol{h}}^{\boldsymbol{k}}-\overline{\boldsymbol{w}}_{\boldsymbol{h}}^{\boldsymbol{k}}\right)(\boldsymbol{y})=\sum_{i=1}^{5} \mathcal{E}_{i}(\boldsymbol{y})
$$


where

$$
\begin{gathered}
\mathcal{E}_{1}(\boldsymbol{y})=\left(\mathbf{Y}^{k+1}-\mathbf{I d}\right)\left(\left[\boldsymbol{\nabla} \mathcal{A}\left(t ; t^{k+1}, \mathbf{X}^{k+1}(\boldsymbol{y})\right)\right]^{-1}(\boldsymbol{u}-\boldsymbol{w})\left(\mathcal{A}\left(t ; t^{k+1}, \mathbf{X}^{k+1}(\boldsymbol{y})\right), t\right)\right), \\
\mathcal{E}_{2}(\boldsymbol{y})=\left[\boldsymbol{\nabla} \mathcal{A}\left(t ; t^{k+1}, \mathbf{X}^{k+1}(\boldsymbol{y})\right)\right]^{-1}(\boldsymbol{u}-\boldsymbol{w})\left(\mathcal{A}\left(t ; t^{k+1}, \mathbf{X}^{k+1}(\boldsymbol{y})\right), t\right) \\
-\left[\boldsymbol{\nabla} \mathcal{A}\left(t^{k} ; t^{k+1}, \mathbf{X}^{k+1}(\boldsymbol{y})\right)\right]^{-1}(\boldsymbol{u}-\boldsymbol{w})\left(\mathcal{A}\left(t^{k} ; t^{k+1}, \mathbf{X}^{k+1}(\boldsymbol{y})\right), t^{k}\right), \\
\mathcal{E}_{3}(\boldsymbol{y})=\left(\left[\boldsymbol{\nabla} \widehat{\mathcal{A}}^{k} \circ\left(\widehat{\mathcal{A}}^{k+1}\right)^{-1}(\boldsymbol{y})\right]^{-1}-\left[\boldsymbol{\nabla} \mathcal{A}_{h}^{k+1, k}(\boldsymbol{y})\right]^{-1}\right)\left(\mathbf{U}^{k}-\mathbf{W}^{k}\right)\left(\widehat{\mathcal{A}}^{k} \circ\left(\widehat{\mathcal{A}}^{k+1}\right)^{-1}(\boldsymbol{y})\right), \\
\mathcal{E}_{4}(\boldsymbol{y})=\left[\boldsymbol{\nabla} \mathcal{A}_{h}^{k+1, k}(\boldsymbol{y})\right]^{-1}\left(\left(\mathbf{U}^{k}-\mathbf{W}^{k}\right)\left(\widehat{\mathcal{A}}^{k} \circ\left(\widehat{\mathcal{A}}^{k+1}\right)^{-1}(\boldsymbol{y})\right)-\left(\mathbf{U}^{k}-\mathbf{W}^{k}\right)\left(\mathcal{A}_{h}^{k+1, k}(\boldsymbol{y})\right)\right), \\
\mathcal{E}_{5}(\boldsymbol{y})=\left[\boldsymbol{\nabla} \mathcal{A}_{h}^{k+1, k}(\boldsymbol{y})\right]^{-1}\left(\left(\mathbf{U}^{k}-\mathbf{W}^{k}\right)-\left(\boldsymbol{u}_{h}^{k}-\boldsymbol{w}_{h}^{k}\right)\right)\left(\mathcal{A}_{h}^{k+1, k}(\boldsymbol{y})\right) .
\end{gathered}
$$

First, we deduce from Lemma 4.1 that

$$
\int_{t^{k}}^{t^{k+1}}\left\|\mathcal{E}_{1}\right\|_{\mathrm{L}^{2}(\mathcal{O})^{2}} \mathrm{~d} t \leqslant C(\delta t)\left\|\mathbf{Y}^{k+1}-\mathbf{I d}\right\|_{\mathrm{L}^{\infty}(\mathcal{O})^{2}} \leqslant C(\delta t)\left(\left|\boldsymbol{\zeta}\left(t^{k}\right)-\boldsymbol{\zeta}_{h}^{k}\right|+\left|\theta\left(t^{k}\right)-\theta_{h}^{k}\right|\right) .
$$

We infer from (3.18), (5.2), (5.3) and Lemma 4.1 that the family of mappings

$$
(\boldsymbol{y}, t) \mapsto\left[\nabla \mathcal{A}\left(t ; t^{k+1}, \mathbf{X}^{k+1}(\boldsymbol{y})\right)\right]^{-1}(\boldsymbol{u}-\boldsymbol{w})\left(\mathcal{A}\left(t ; t^{k+1}, \mathbf{X}^{k+1}(\boldsymbol{y})\right), t\right)
$$

is bounded in $\mathrm{W}^{1, \infty}\left(0, T ; \mathrm{L}^{\infty}(\mathcal{O})^{2}\right)$ with respect to $k$ and we thus find that

$$
\int_{t^{k}}^{t^{k+1}}\left\|\mathcal{E}_{2}\right\|_{\mathrm{L}^{2}(\mathcal{O})^{2}} \mathrm{~d} t \leqslant C(\delta t)^{2}
$$

For the next term, the use of (3.18), (5.3), (5.6) and Lemmata 4.1 and 5.5 yields

$$
\int_{t^{k}}^{t^{k+1}}\left\|\mathcal{E}_{3}\right\|_{L^{2}(\mathcal{O})^{2}} \mathrm{~d} t \leqslant C(\delta t)\left(\left|\boldsymbol{\zeta}\left(t^{k}\right)-\boldsymbol{\zeta}_{h}^{k}\right|+\left|\theta\left(t^{k}\right)-\theta_{h}^{k}\right|+h^{1 / 2}\right) .
$$

Using assumption (2.17), it can be shown that $\left\|\left[\nabla \mathcal{A}_{h}^{k+1, k}\right]^{-1}\right\|_{L^{\infty}(\mathcal{O})^{4}}$ is uniformly bounded with respect to $k$ and $h$ (see the proof of Lem. 5.4 for details). In addition, using the regularity hypotheses, we obtain that $\left\|\nabla\left(\mathbf{U}^{k}-\mathbf{W}^{k}\right)\right\|_{\mathrm{L}^{\infty}(\mathcal{O})^{4}}$ is also uniformly bounded with respect to $k$ and $h$. Therefore, we have

$$
\int_{t^{k}}^{t^{k+1}}\left\|\mathcal{E}_{4}\right\|_{L^{2}(\mathcal{O})^{2}} \mathrm{~d} t \leqslant C(\delta t)\left\|\widehat{\mathcal{A}}^{k} \circ\left(\widehat{\mathcal{A}}^{k+1}\right)^{-1}-\mathcal{A}_{h}^{k+1, k}\right\|_{L^{\infty}(\mathcal{O})^{2}}
$$

which in turn implies

$$
\int_{t^{k}}^{t^{k+1}}\left\|\mathcal{E}_{4}\right\|_{\mathrm{L}^{2}(\mathcal{O})^{2}} \mathrm{~d} t \leqslant C(\delta t)\left(\left\|\hat{\mathcal{A}}^{k}-\mathcal{A}_{h}^{k}\right\|_{\mathrm{L}^{\infty}(\mathcal{O})^{2}}+\left\|\left(\widehat{\mathcal{A}}^{k+1}\right)^{-1}-\left(\mathcal{A}_{h}^{k+1}\right)^{-1}\right\|_{\mathrm{L}^{\infty}(\mathcal{O})^{2}}\right) .
$$

Hence, using Lemma 5.2, we obtain

$$
\int_{t^{k}}^{t^{k+1}}\left\|\mathcal{E}_{4}\right\|_{\mathrm{L}^{2}(\mathcal{O})^{2}} \mathrm{~d} t \leqslant C(\delta t)\left(\left|\boldsymbol{\zeta}\left(t^{k}\right)-\boldsymbol{\zeta}_{h}^{k}\right|+\left|\theta\left(t^{k}\right)-\theta_{h}^{k}\right|+h\right) .
$$


Finally, using a change of variables, we reach

$$
\int_{t^{k}}^{t^{k+1}}\left\|\mathcal{E}_{5}\right\|_{\mathrm{L}^{2}(\mathcal{O})^{2}} \mathrm{~d} t \leqslant C(\delta t)\left(\left\|\mathbf{U}^{k}-\boldsymbol{u}_{h}^{k}\right\|_{\mathrm{L}^{2}(\mathcal{O})^{2}}+\left\|\mathbf{W}^{k}-\boldsymbol{w}_{h}^{k}\right\|_{\mathrm{L}^{2}(\mathcal{O})^{2}}\right)
$$

and infer from Lemma 5.6 that

$$
\int_{t^{k}}^{t^{k+1}}\left\|\mathcal{E}_{5}\right\|_{\mathrm{L}^{2}(\mathcal{O})^{2}} \mathrm{~d} t \leqslant C(\delta t)\left(\left\|\mathbf{U}^{k}-\boldsymbol{u}_{h}^{k}\right\|_{\mathrm{L}^{2}(\mathcal{O})^{2}}+\left|\boldsymbol{\zeta}\left(t^{k}\right)-\boldsymbol{\zeta}_{h}^{k}\right|+\left|\theta\left(t^{k}\right)-\theta_{h}^{k}\right|+\delta t+h\right) .
$$

Collecting all these bounds then establishes the lemma.

Proof of Theorem 6.1. From definition (4.5), we have

$$
\mathcal{C}^{k}-\mathcal{A}_{h}^{k+1, k} \circ \mathcal{B}_{h}^{k}=\mathbf{Y}^{k} \circ \mathcal{A}\left(t^{k} ; t^{k+1}, \cdot\right) \circ \mathcal{B}\left(t^{k} ; t^{k+1}, \cdot\right) \circ \mathbf{X}^{k+1}-\mathcal{A}_{h}^{k+1, k} \circ \mathcal{B}_{h}^{k}
$$

and it follows from (5.5) and (6.5) that

$$
\begin{aligned}
\mathcal{C}^{k}-\mathcal{A}_{h}^{k+1, k} \circ \mathcal{B}_{h}^{k} & =\mathbf{Y}^{k} \circ \mathcal{A}\left(t^{k} ; t^{k+1}, \cdot\right) \circ \mathbf{X}^{k+1} \circ \mathbf{Y}^{k+1} \circ \mathcal{B}\left(t^{k} ; t^{k+1}, \cdot\right) \circ \mathbf{X}^{k+1}-\mathcal{A}_{h}^{k+1, k} \circ \mathcal{B}_{h}^{k} \\
& =\widehat{\mathcal{A}}^{k} \circ\left(\widehat{\mathcal{A}}^{k+1}\right)^{-1} \circ \widehat{\mathcal{B}}^{k}-\mathcal{A}_{h}^{k} \circ\left(\mathcal{A}_{h}^{k+1}\right)^{-1} \circ \mathcal{B}_{h}^{k} .
\end{aligned}
$$

The right-hand side of the above equality is then broken into two contributions:

$$
\begin{aligned}
\mathcal{C}^{k}-\mathcal{A}_{h}^{k+1, k} \circ \mathcal{B}_{h}^{k}=\widehat{\mathcal{A}}^{k} \circ\left(\widehat{\mathcal{A}}^{k+1}\right)^{-1} \circ \widehat{\mathcal{B}}^{k}-\widehat{\mathcal{A}}^{k} \circ\left(\widehat{\mathcal{A}}^{k+1}\right)^{-1} \circ \mathcal{B}_{h}^{k} & +\widehat{\mathcal{A}}^{k} \circ\left(\widehat{\mathcal{A}}^{k+1}\right)^{-1} \circ \mathcal{B}_{h}^{k}-\mathcal{A}_{h}^{k} \circ\left(\mathcal{A}_{h}^{k+1}\right)^{-1} \circ \mathcal{B}_{h}^{k} .
\end{aligned}
$$

Using (5.6) and (5.7), we have that

$$
\left\|\widehat{\mathcal{A}}^{k} \circ\left(\widehat{\mathcal{A}}^{k+1}\right)^{-1} \circ \widehat{\mathcal{B}}^{k}-\widehat{\mathcal{A}}^{k} \circ\left(\widehat{\mathcal{A}}^{k+1}\right)^{-1} \circ \mathcal{B}_{h}^{k}\right\|_{\mathrm{L}^{2}(\mathcal{O})^{2}} \leqslant C\left\|\widehat{\mathcal{B}}^{k}-\mathcal{B}_{h}^{k}\right\|_{\mathrm{L}^{2}(\mathcal{O})^{2}} \text {. }
$$

Additionally, taking the difference between problems (6.6) and (3.14), we find that, for all $t$ in $\left[t^{k}, t^{k+1}\right]$,

$$
\begin{aligned}
& \left\|\widehat{\mathcal{B}}\left(t ; t^{k+1}, \cdot\right)-\mathcal{B}_{h}\left(t ; t^{k+1}, \cdot\right)\right\|_{L^{2}(\mathcal{O})^{2}} \leqslant \\
& \int_{t}^{t^{k+1}}\left\|\nabla\left(\mathbf{Y}^{k+1} \circ(\overline{\boldsymbol{u}}-\overline{\boldsymbol{w}})(\cdot, s) \circ \mathbf{X}^{k+1}\right)\right\|_{L^{\infty}(\mathcal{O})^{4}}\left\|\widehat{\mathcal{B}}\left(s ; t^{k+1}, \cdot\right)-\boldsymbol{\mathcal { B }}_{h}\left(s ; t^{k+1}, \cdot\right)\right\|_{L^{2}(\mathcal{O})^{2}} \mathrm{~d} s \\
& \quad+\int_{t}^{t^{k+1}}\left\|\left(\mathbf{Y}^{k+1} \circ(\overline{\boldsymbol{u}}-\overline{\boldsymbol{w}})(\cdot, s) \circ \mathbf{X}^{k+1}-\left(\overline{\boldsymbol{u}}_{\boldsymbol{h}}^{\boldsymbol{k}}-\overline{\boldsymbol{w}}_{\boldsymbol{h}}^{\boldsymbol{k}}\right)\right) \circ \boldsymbol{\mathcal { B }}_{h}\left(s ; t^{k+1}, \cdot\right)\right\|_{L^{2}(\mathcal{O})^{2}} \mathrm{~d} s .
\end{aligned}
$$

Combining the above inequality with Grönwall's inequality and the fact that the Jacobian of $\mathcal{B}_{h}$ is bounded (owing to assumption (6.2)), we obtain

$$
\left\|\widehat{\mathcal{B}}\left(t ; t^{k+1}, \cdot\right)-\mathcal{B}_{h}\left(t ; t^{k+1}, \cdot\right)\right\|_{L^{2}(\mathcal{O})^{2}} \leqslant C \int_{t^{k}}^{t^{k+1}}\left\|\mathbf{Y}^{k+1} \circ(\overline{\boldsymbol{u}}-\overline{\boldsymbol{w}})(\cdot, s) \circ \mathbf{X}^{k+1}-\left(\overline{\boldsymbol{u}}_{\boldsymbol{h}}^{\boldsymbol{k}}-\overline{\boldsymbol{w}}_{\boldsymbol{h}}^{\boldsymbol{k}}\right)\right\|_{\mathrm{L}^{2}(\mathcal{O})^{2}} \mathrm{~d} s
$$

and, using Lemma 6.2, finally deduce that

$$
\left\|\widehat{\mathcal{B}}\left(t^{k} ; t^{k+1}, \cdot\right)-\mathcal{B}_{h}^{k}\right\|_{L^{2}(\mathcal{O})^{2}} \leqslant C(\delta t)\left(\left\|\mathbf{U}^{k}-\boldsymbol{u}_{h}^{k}\right\|_{L^{2}(\mathcal{O})^{2}}+\left|\boldsymbol{\zeta}\left(t^{k}\right)-\boldsymbol{\zeta}_{h}^{k}\right|+\left|\theta\left(t^{k}\right)-\theta_{h}^{k}\right|+\delta t+h^{1 / 2}\right) .
$$


We now turn our attention on the second term in the right-hand side of (6.7). Since the Jacobian of $\boldsymbol{B}_{h}^{k}$ is bounded, we find that

$$
\left\|\hat{\mathcal{A}}^{k} \circ\left(\hat{\mathcal{A}}^{k+1}\right)^{-1} \circ \boldsymbol{B}_{h}^{k}-\mathcal{A}_{h}^{k} \circ\left(\mathcal{A}_{h}^{k+1}\right)^{-1} \circ \boldsymbol{B}_{h}^{k}\right\|_{L^{2}(\mathcal{O})^{2}} \leqslant C\left\|\widehat{\mathcal{A}}^{k} \circ\left(\hat{\mathcal{A}}^{k+1}\right)^{-1}-\mathcal{A}_{h}^{k} \circ\left(\mathcal{A}_{h}^{k+1}\right)^{-1}\right\|_{L^{2}(\mathcal{O})^{2}}
$$

Given the bound obtained in Lemma 5.7, we have

$$
\begin{aligned}
\left\|\widehat{\mathcal{A}}^{k} \circ\left(\widehat{\mathcal{A}}^{k+1}\right)^{-1} \circ \mathcal{B}_{h}^{k}-\mathcal{A}_{h}^{k} \circ\left(\mathcal{A}_{h}^{k+1}\right)^{-1} \circ \boldsymbol{B}_{h}^{k}\right\|_{L^{2}(\mathcal{O})^{2}} \leqslant & \\
& C(\delta t)\left(\left|\boldsymbol{\zeta}\left(t^{k}\right)-\boldsymbol{\zeta}_{h}^{k}\right|+\left|\theta\left(t^{k}\right)-\theta_{h}^{k}\right|+\left|\boldsymbol{\xi}\left(t^{k}\right)-\boldsymbol{\xi}_{h}^{k}\right|+\left|\omega\left(t^{k}\right)-\omega_{h}^{k}\right|+\delta t+h\right) .
\end{aligned}
$$

Collecting (6.7), (6.8), (6.9) and this last inequality therefore yields estimate (6.4).

Lemma 6.3. Assume that $h$ is small enough. Then, for all $k$ in $\{0, \ldots, N-1\}$ and $\mu$ in $[0,1]$, the mapping

$$
\mu \mathcal{C}^{k}+(1-\mu) \mathcal{A}_{h}^{k+1, k} \circ \mathcal{B}_{h}^{k}: \mathcal{O} \rightarrow \mathcal{O}
$$

is a diffeomorphism with Jacobian satisfying (5.8) for some positive constant c.

Proof. It suffices to write

$$
\mu \mathcal{C}^{k}+(1-\mu) \mathcal{A}_{h}^{k+1, k} \circ \mathcal{B}_{h}^{k}=\mu \widehat{\mathcal{A}}^{k} \circ\left(\widehat{\mathcal{A}}^{k+1}\right)^{-1} \circ \widehat{\mathcal{B}}^{k}+(1-\mu) \mathcal{A}_{h}^{k} \circ\left(\mathcal{A}_{h}^{k+1}\right)^{-1} \circ \mathcal{B}_{h}^{k},
$$

and follow the proof of Lemma 5.4 using assumptions (6.1) and (6.2).

Lemma 6.4. For all $k$ in $\{0, \ldots, N\}$ and $\mu$ in $[0,1]$, there exists a constant $C$ such that

$$
\left\|\mathcal{C}^{k}-\mathbf{I d}\right\|_{L^{\infty}(\mathcal{O})^{2}} \leqslant C(\delta t)\left(1+\left|\theta\left(t^{k}\right)-\theta_{h}^{k}\right|+\left|\boldsymbol{\xi}\left(t^{k}\right)-\boldsymbol{\xi}_{h}^{k}\right|+\left|\omega\left(t^{k}\right)-\omega_{h}^{k}\right|\right) .
$$

Proof. For all $t$ in $\left[t^{k}, t^{k+1}\right]$, define $\widehat{\mathcal{C}}\left(t ; t^{k+1}, \cdot\right)=\mathbf{Y}^{k} \circ \mathcal{C}\left(t ; t^{k+1}, \cdot\right) \circ \mathbf{X}^{k+1}$, hence $\widehat{\mathcal{C}}\left(t^{k} ; t^{k+1}, \cdot\right)=\mathcal{C}^{k}$. It is deduced from problem (2.8) that, for all $\boldsymbol{x}$ in $\mathcal{O}$, the function $\widehat{\mathcal{C}}\left(\cdot ; t^{k+1}, \boldsymbol{x}\right)$ satisfies the following initial value problem

$$
\left\{\begin{array}{l}
\frac{\partial \widehat{\mathcal{C}}}{\partial t}\left(t ; t^{k+1}, \boldsymbol{x}\right)=J_{\mathbf{Y}^{k}}\left(\mathbf{X}^{k}\left(\widehat{\mathcal{C}}\left(t ; t^{k+1}, \boldsymbol{x}\right)\right)\right) \boldsymbol{u}\left(\mathbf{X}^{k}\left(\widehat{\mathcal{C}}\left(t ; t^{k+1}, \boldsymbol{x}\right)\right), t\right), \\
\widehat{\mathcal{C}}\left(t^{k+1} ; t^{k+1}, \boldsymbol{x}\right)=\mathbf{Y}^{k} \circ \mathbf{X}^{k+1}(\boldsymbol{x}) .
\end{array}\right.
$$

The Taylor-Lagrange inequality combined with Lemma 4.1 and regularity assumption (3.18) then leads to

$$
\left\|\mathcal{C}^{k}-\mathbf{I} \mathbf{d}\right\|_{\mathrm{L}^{\infty}(\mathcal{O})^{2}} \leqslant\left\|\mathbf{Y}^{k} \circ \mathbf{X}^{k+1}-\mathbf{I} \mathbf{d}\right\|_{L^{\infty}(\mathcal{O})^{2}}+C(\delta t),
$$

from which the result is inferred using Lemma 4.3.

\section{Proof of the MAIN RESUlt}

In this section, it is assumed that every assumption under which Theorem 3.5 is formulated holds. In particular, the quantities $\delta t$ and $h$ are both smaller than unity and we take advantage of this fact to simplify the estimates.

We will now show that the exact strong solution of (1.1)-(1.8) satisfies at each instant $t^{k}$ a perturbed system of semidiscretized equations which leads to a weak formulation similar to problem (3.16)-(3.17), and give estimates 
for the perturbations. Consider $(\boldsymbol{u}, \boldsymbol{\zeta}, \theta, p)$ the solution to (1.1)-(1.8) and assume that it satisfies (3.18). For any $k$ in $\{0, \ldots, N-1\}$, we have that

$$
\begin{gathered}
\frac{\boldsymbol{u}\left(\cdot, t^{k+1}\right)-\boldsymbol{u}\left(\mathcal{C}\left(t^{k} ; t^{k+1}, \cdot\right), t^{k}\right)}{\delta t}-\nu \Delta \boldsymbol{u}\left(\cdot, t^{k+1}\right)+\nabla p\left(\cdot, t^{k+1}\right)=\boldsymbol{f}\left(\cdot, t^{k+1}\right)+\boldsymbol{e}_{d_{t} \boldsymbol{u}}^{k} \text { in } \mathcal{F}\left(\boldsymbol{\zeta}\left(t^{k+1}\right), \theta\left(t^{k+1}\right)\right), \\
M \frac{\boldsymbol{\xi}\left(t^{k+1}\right)-\boldsymbol{\xi}\left(t^{k}\right)}{\delta t}=-\int_{\partial \mathcal{S}\left(\boldsymbol{\zeta}\left(t^{k+1}\right), \theta\left(t^{k+1}\right)\right)} \boldsymbol{\sigma}(\boldsymbol{u}, p) \boldsymbol{n}\left(\boldsymbol{x}, t^{k+1}\right) \mathrm{d} \Gamma+\boldsymbol{f}_{M}\left(t^{k+1}\right)+\boldsymbol{e}_{\xi}^{k} \\
I \frac{\omega\left(t^{k+1}\right)-\omega\left(t^{k}\right)}{\delta t}=-\int_{\partial \mathcal{S}\left(\boldsymbol{\zeta}\left(t^{k+1}\right), \theta\left(t^{k+1}\right)\right)}\left(\boldsymbol{x}-\boldsymbol{\zeta}_{h}^{k+1}\right)^{\perp} \cdot \boldsymbol{\sigma}(\boldsymbol{u}, p) \boldsymbol{n}\left(\boldsymbol{x}, t^{k+1}\right) \mathrm{d} \Gamma+f_{I}\left(t^{k+1}\right)+e_{\omega}^{k}
\end{gathered}
$$

where

$$
\boldsymbol{e}_{d_{t} \boldsymbol{u}}^{k}=\frac{\boldsymbol{u}\left(\cdot, t^{k+1}\right)-\boldsymbol{u}\left(\mathcal{C}\left(t^{k} ; t^{k+1}, \cdot\right), t^{k}\right)}{\delta t}-\frac{\mathrm{d}}{\mathrm{d} t}[\boldsymbol{u} \circ \mathcal{C}]\left(\cdot, t^{k+1}\right),
$$

and

$$
\boldsymbol{e}_{\boldsymbol{\xi}}^{k}=\frac{\boldsymbol{\xi}\left(t^{k+1}\right)-\boldsymbol{\xi}\left(t^{k}\right)}{\delta t}-\boldsymbol{\xi}^{\prime}\left(t^{k+1}\right), \quad e_{\omega}^{k}=\frac{\omega\left(t^{k+1}\right)-\omega\left(t^{k}\right)}{\delta t}-\omega^{\prime}\left(t^{k+1}\right)
$$

Using regularity assumptions (3.18) and the Taylor-Lagrange inequality, we can prove the following result.

Lemma 7.1. For all $k$ in $\{0, \ldots, N-1\}$, there exists a constant $C$ such that the quantities $\boldsymbol{e}_{d_{t} \boldsymbol{u}}^{k}$, $\boldsymbol{e}_{\xi}^{k}$ and $\boldsymbol{e}_{\omega}^{k}$ defined above satisfy the following inequalities

$$
\left\|\boldsymbol{e}_{d_{t} \boldsymbol{u}}^{k}\right\|_{L^{2}\left(\mathcal{F}\left(\boldsymbol{\zeta}\left(t^{k+1}\right), \theta\left(t^{k+1}\right)\right)\right)^{2}} \leqslant C(\delta t), \quad\left|\boldsymbol{e}_{\boldsymbol{\xi}}^{k}\right| \leqslant C(\delta t) \quad \text { and }\left|e_{\omega}^{k}\right| \leqslant C(\delta t) .
$$

The change of variable introduced in Section 4 allows to transform equations (7.1) to (7.3), set at instant $t^{k+1}$ in a domain in which the rigid body has $\zeta\left(t^{k+1}\right)$ as the position for its center of mass and $\theta\left(t^{k+1}\right)$ as its orientation, into a system of equations set in a domain where the solid is centered on $\boldsymbol{\zeta}_{h}^{k+1}$ with orientation $\theta_{h}^{k+1}$. This step permits the subsequent comparison between the exact and approximate solutions of the problem. We suppose that (4.1) holds so that we can consider the change of variables $\mathbf{X}^{k+1}$. Retaining the notations from Section 4 , we obtain that the couple $\left(\mathbf{U}^{k+1}, \mathrm{P}^{k+1}\right)$ satisfies

$$
\frac{\mathbf{U}^{k+1}-\left(J_{\mathbf{Y}^{k+1}} \circ \mathbf{X}^{k+1}\right)\left(J_{\mathbf{X}^{k}} \circ \mathcal{C}^{k}\right)\left(\mathbf{U}^{k} \circ \mathcal{C}^{k}\right)}{\delta t}-\nu\left[L^{k+1} \mathbf{U}^{k+1}\right]+\left[\boldsymbol{G}^{k+1} \mathrm{P}^{k+1}\right]=\mathbf{F}^{k+1}+\mathbf{E}_{d_{t} \boldsymbol{u}}^{k} \text { in } \mathcal{F}\left(\boldsymbol{\zeta}_{h}^{k+1}, \theta_{h}^{k+1}\right),
$$

where $\mathbf{F}^{k+1}(\boldsymbol{y})=J_{\mathbf{Y}^{k+1}}\left(\mathbf{X}^{k+1}(\boldsymbol{y})\right) \boldsymbol{f}\left(\mathbf{X}^{k+1}(\boldsymbol{y}), t^{k+1}\right), \mathbf{E}_{d_{t} \boldsymbol{u}}^{k}(\boldsymbol{y})=J_{\mathbf{Y}^{k+1}}\left(\mathbf{X}^{k+1}(\boldsymbol{y})\right) \boldsymbol{e}_{d_{t} \boldsymbol{u}}^{k}\left(\mathbf{X}^{k+1}(\boldsymbol{y})\right)$, and, from property (4.6),

$$
\operatorname{div} \mathbf{U}^{k+1}=0 \text { in } \mathcal{F}\left(\boldsymbol{\zeta}_{h}^{k+1}, \theta_{h}^{k+1}\right) .
$$

The transformed equations for the rigid solid are obtained using property (4.4) and we have

$$
\begin{gathered}
M \frac{\boldsymbol{\Xi}^{k+1}-\boldsymbol{\Xi}^{k}}{\delta t}=-\int_{\partial \mathcal{S}\left(\boldsymbol{\zeta}_{h}^{k+1}, \theta_{h}^{k+1}\right)} \boldsymbol{\sigma}\left(\mathbf{U}^{k+1}, \mathrm{P}^{k+1}\right) \boldsymbol{n} \mathrm{d} \Gamma+\mathbf{F}_{M}^{k+1}+\mathbf{E}_{\boldsymbol{\xi}}^{k}, \\
I \frac{\Omega^{k+1}-\Omega^{k}}{\delta t}=-\int_{\partial \mathcal{S}\left(\boldsymbol{\zeta}_{h}^{k+1}, \theta_{h}^{k+1}\right)}\left(\boldsymbol{x}-\boldsymbol{\zeta}_{h}^{k+1}\right)^{\perp} \cdot \boldsymbol{\sigma}\left(\mathbf{U}^{k+1}, \mathrm{P}^{k+1}\right) \boldsymbol{n} \mathrm{d} \Gamma+\mathrm{F}_{I}^{k+1}+\mathrm{E}_{\omega}^{k},
\end{gathered}
$$

where $\mathbf{F}_{M}^{k+1}=\mathbf{R}_{\theta_{h}^{k+1}-\theta\left(t^{k+1}\right)} \boldsymbol{f}_{M}\left(t^{k+1}\right), \mathrm{F}_{I}^{k+1}=f_{I}^{k+1}, \mathbf{E}_{\boldsymbol{\xi}}^{k}=\mathbf{R}_{\theta_{h}^{k}-\theta\left(t^{k}\right)} \boldsymbol{e}_{\boldsymbol{\xi}}^{k}$ and $\mathrm{E}_{\omega}^{k}=e_{\omega}^{k}$. 
Observe that, while $\mathcal{V}_{h}^{k+1}$ is not a subspace of $\mathcal{V}\left(\boldsymbol{\zeta}_{h}^{k+1}, \theta_{h}^{k+1}\right)$ due to the nonconforming approximation of the fluid domain, any function $\left(\boldsymbol{v}_{h}^{k+1}, \boldsymbol{\xi}_{\boldsymbol{v}_{h}^{k+1}}, \omega_{\boldsymbol{v}_{h}^{k+1}}\right)$ of $\mathcal{V}_{h}^{k+1}$ can be easily extended into a function of $\mathrm{H}_{0}^{1}(\mathcal{O})^{2} \times \mathbb{R}^{3}$ by setting

$$
\boldsymbol{v}_{h}^{k+1}(\boldsymbol{x})=\boldsymbol{\xi}_{\boldsymbol{v}_{h}^{k+1}}+\omega_{\boldsymbol{v}_{h}^{k+1}}\left(\boldsymbol{x}-\boldsymbol{\zeta}_{h}^{k+1}\right)^{\perp}, \forall \boldsymbol{x} \in \mathcal{S}_{h}^{k+1}
$$

Similarly, any function $q_{h}^{k+1}$ of $\mathcal{Q}_{h}^{k+1}$ can be extended into a function of $\mathrm{L}_{0}^{2}(\mathcal{O})$ by setting $q_{h}^{k+1}=0$ in $\mathcal{S}_{h}^{k+1}$. We therefore introduce the couple of "global" discretization spaces, both defined over the triangulation $\widetilde{\mathscr{T}}_{h}^{k+1}$,

$$
\begin{array}{r}
\hat{\mathcal{V}}_{h}^{k+1}=\left\{\left(\boldsymbol{v}_{h}, \boldsymbol{\xi}_{\boldsymbol{v}_{h}}, \omega_{\boldsymbol{v}_{h}}\right) \in \mathrm{C}(\overline{\mathcal{O}})^{2} \cap \mathrm{H}_{0}^{1}(\mathcal{O})^{2} \times \mathbb{R}^{3} ; \boldsymbol{v}_{\left.h\right|_{K}} \in\left[\mathbb{P}_{1}(K) \oplus\left\langle\lambda_{1} \lambda_{2} \lambda_{3}\right\rangle\right]^{2}, \forall K \in \widetilde{\mathscr{T}}_{h}^{k+1},\right. \\
\text { and } \left.\boldsymbol{v}_{h}(\boldsymbol{x})=\boldsymbol{\xi}_{\boldsymbol{v}_{h}}+\omega_{\boldsymbol{v}_{h}}\left(\boldsymbol{x}-\boldsymbol{\zeta}_{h}^{k+1}\right)^{\perp}, \forall \boldsymbol{x} \in \mathcal{S}_{h}^{k+1}\right\}
\end{array}
$$

and $\hat{\mathcal{Q}}_{h}^{k+1}=\left\{q_{h} \in \mathrm{C}(\overline{\mathcal{O}}) \cap \mathrm{L}_{0}^{2}(\mathcal{O}) ;\left.q_{h}\right|_{K} \in \mathbb{P}_{1}(K), \forall K \in \widetilde{\mathscr{T}}_{h}^{k+1}\right.$, and $q_{h}=0$ in $\left.\mathcal{S}_{h}^{k+1}\right\}$, and their respective continuous counterparts

$$
\hat{\mathcal{V}}\left(\boldsymbol{\zeta}_{h}^{k+1}, \theta_{h}^{k+1}\right)=\left\{\left(\boldsymbol{v}, \boldsymbol{\xi}_{\boldsymbol{v}}, \omega_{\boldsymbol{v}}\right) \in \mathrm{C}(\overline{\mathcal{O}})^{2} \cap \mathrm{H}_{0}^{1}(\mathcal{O})^{2} \times \mathbb{R}^{3} ; \boldsymbol{v}(\boldsymbol{x})=\boldsymbol{\xi}_{\boldsymbol{v}}+\omega_{\boldsymbol{v}}\left(\boldsymbol{x}-\boldsymbol{\zeta}_{h}^{k+1}\right)^{\perp}, \forall \boldsymbol{x} \in \mathcal{S}\left(\boldsymbol{\zeta}_{h}^{k+1}, \theta_{h}^{k+1}\right)\right\}
$$

and $\hat{\mathcal{Q}}\left(\boldsymbol{\zeta}_{h}^{k+1}, \theta_{h}^{k+1}\right)=\left\{q \in \mathrm{C}(\overline{\mathcal{O}}) \cap \mathrm{L}_{0}^{2}(\mathcal{O}) ; q=0\right.$ in $\left.\mathcal{S}\left(\boldsymbol{\zeta}_{h}^{k+1}, \theta_{h}^{k+1}\right)\right\}$. Then, multiplying scalarly equation (7.5)

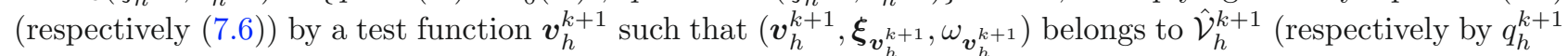
in $\left.\hat{\mathcal{Q}}_{h}^{k+1}\right)$, integrating over the domain $\mathcal{F}\left(\boldsymbol{\zeta}_{h}^{k+1}, \theta_{h}^{k+1}\right)$ and using equations (7.7) and (7.8), we obtain that the quadruplet $\left(\mathbf{U}^{k+1}, \mathbf{\Xi}^{k+1}, \Omega^{k+1}, \mathrm{P}^{k+1}\right)$ is solution to the following variational problem

$$
\begin{aligned}
& \int_{\mathcal{F}\left(\boldsymbol{\zeta}_{h}^{k+1}, \theta_{h}^{k+1}\right)}\left(\frac{\mathbf{U}^{k+1}-\mathbf{U}^{k} \circ \mathcal{C}^{k}}{\delta t}\right) \cdot \boldsymbol{v}_{h}^{k+1} \mathrm{~d} \boldsymbol{x}+M \frac{\boldsymbol{\Xi}^{k+1}-\boldsymbol{\Xi}^{k}}{\delta t} \cdot \boldsymbol{\xi}_{\boldsymbol{v}_{h}^{k+1}}+I \frac{\Omega^{k+1}-\Omega^{k}}{\delta t} \omega_{\boldsymbol{v}_{h}^{k+1}} \\
& +2 \nu \int_{\mathcal{F}\left(\boldsymbol{\zeta}_{h}^{k+1}, \theta_{h}^{k+1}\right)} \mathbf{D}\left(\mathbf{U}^{k+1}\right): \mathbf{D}\left(\boldsymbol{v}_{h}^{k+1}\right) \mathrm{d} \boldsymbol{x}-\int_{\mathcal{F}\left(\boldsymbol{\zeta}_{h}^{k+1}, \theta_{h}^{k+1}\right)} \mathrm{P}^{k+1} \operatorname{div} \boldsymbol{v}_{h}^{k+1} \mathrm{~d} \boldsymbol{x} \\
& =\int_{\mathcal{F}\left(\boldsymbol{\zeta}_{h}^{k+1}, \theta_{h}^{k+1}\right)} \mathbf{F}^{k+1} \cdot \boldsymbol{v}_{h}^{k+1} \mathrm{~d} \boldsymbol{x}+\mathbf{F}_{M}^{k+1} \cdot \boldsymbol{\xi}_{\boldsymbol{v}_{h}^{k+1}}+\mathrm{F}_{I}^{k+1} \omega_{\boldsymbol{v}_{h}^{k+1}} \\
& +\int_{\mathcal{F}\left(\boldsymbol{\zeta}_{h}^{k+1}, \theta_{h}^{k+1}\right)}\left(\mathbf{E}_{d_{t} \boldsymbol{u}}^{k}+\mathbf{E}_{\mathrm{ch}}^{k}\right) \cdot \boldsymbol{v}_{h}^{k+1} \mathrm{~d} \boldsymbol{x}+\mathbf{E}_{\boldsymbol{\xi}}^{k} \cdot \boldsymbol{\xi}_{\boldsymbol{v}_{h}^{k+1}}+\mathrm{E}_{\omega}^{k} \omega_{\boldsymbol{v}_{h}^{k+1}}, \forall\left(\boldsymbol{v}_{h}^{k+1}, \boldsymbol{\xi}_{\left.\boldsymbol{v}_{h}^{k+1}, \omega_{\boldsymbol{v}_{h}^{k+1}}\right) \in \hat{\mathcal{V}}_{h}^{k+1},},\right. \\
& -\int_{\mathcal{F}\left(\boldsymbol{\zeta}_{h}^{k+1}, \theta_{h}^{k+1}\right)} q_{h}^{k+1} \operatorname{div} \mathbf{U}^{k+1} \mathrm{~d} \boldsymbol{x}=0, \quad \forall q_{h}^{k+1} \in \hat{\mathcal{Q}}_{h}^{k+1},
\end{aligned}
$$


with

$$
\mathbf{E}_{\mathrm{ch}}^{k}=\frac{\left(\left(J_{\mathbf{Y}^{k+1}} \circ \mathbf{X}^{k+1}\right)\left(J_{\mathbf{X}^{k}} \circ \mathcal{C}^{k}\right)-\mathbf{I d}\right)\left(\mathbf{U}^{k} \circ \mathcal{C}^{k}\right)}{\delta t}+\nu\left(L^{k+1}-\Delta\right) \mathbf{U}^{k+1}+\left(\boldsymbol{G}^{k+1}-\nabla\right) \mathrm{P}^{k+1}
$$

We infer from Lemmata 4.1, 4.3, 4.2 and 6.4 and assumptions (3.18) that

$$
\begin{array}{r}
\left\|\mathbf{E}_{\mathrm{ch}}^{k}\right\|_{L^{2}\left(\mathcal{F}\left(\boldsymbol{\zeta}_{h}^{k+1}, \theta_{h}^{k+1}\right)\right)^{2}} \leqslant C\left(\left|\boldsymbol{\zeta}\left(t^{k+1}\right)-\boldsymbol{\zeta}_{h}^{k+1}\right|+\left|\theta\left(t^{k+1}\right)-\theta_{h}^{k+1}\right|\right)+C\left(1+\left|\boldsymbol{\zeta}\left(t^{k+1}\right)-\boldsymbol{\zeta}_{h}^{k+1}\right|+\left|\theta\left(t^{k+1}\right)-\theta_{h}^{k+1}\right|\right) \\
\left(1+\left|\boldsymbol{\zeta}\left(t^{k}\right)-\boldsymbol{\zeta}_{h}^{k}\right|+\left|\theta\left(t^{k}\right)-\theta_{h}^{k}\right|+\left|\boldsymbol{\xi}\left(t^{k}\right)-\boldsymbol{\xi}_{h}^{k}\right|+\left|\omega\left(t^{k}\right)-\omega_{h}^{k}\right|\right)^{2} \\
\left(\left|\boldsymbol{\zeta}\left(t^{k}\right)-\boldsymbol{\zeta}_{h}^{k}\right|+\left|\theta\left(t^{k}\right)-\theta_{h}^{k}\right|+\left|\boldsymbol{\xi}\left(t^{k}\right)-\boldsymbol{\xi}_{h}^{k}\right|+\left|\omega\left(t^{k}\right)-\omega_{h}^{k}\right|+\delta t\right)
\end{array}
$$

We will now proceed with the finite element analysis of problem (3.16)-(3.17). The particular construction of the domain $\mathcal{F}_{h}^{k+1}$ makes possible the use of an important result relative to the existence of projectors from [29]. First, notice that

$$
\begin{aligned}
2 \nu \int_{\mathcal{F}\left(\boldsymbol{\zeta}_{h}^{k+1}, \theta_{h}^{k+1}\right)} \mathbf{D}\left(\mathbf{U}^{k+1}\right): \mathbf{D}\left(\boldsymbol{v}_{h}^{k+1}\right) \mathrm{d} \boldsymbol{x}-\int_{\mathcal{F}\left(\boldsymbol{\zeta}_{h}^{k+1}, \theta_{h}^{k+1}\right)} \mathrm{P}^{k+1} \operatorname{div} \boldsymbol{v}_{h}^{k+1} \mathrm{~d} \boldsymbol{x} & =2 \nu \int_{\mathcal{O}} \mathbf{D}\left(\mathbf{U}^{k+1}\right): \mathbf{D}\left(\boldsymbol{v}_{h}^{k+1}\right) \mathrm{d} \boldsymbol{x}-\int_{\mathcal{O}} \mathrm{P}^{k+1} \operatorname{div} \boldsymbol{v}_{h}^{k+1} \mathrm{~d} \boldsymbol{x} \\
& -\int_{\mathcal{F}\left(\boldsymbol{\zeta}_{h}^{k+1}, \theta_{h}^{k+1}\right)} q_{h}^{k+1} \operatorname{div} \mathbf{U}^{k+1} \mathrm{~d} \boldsymbol{x}=-\int_{\mathcal{O}} q_{h}^{k+1} \operatorname{div} \mathbf{U}^{k+1} \mathrm{~d} \boldsymbol{x} .
\end{aligned}
$$

Adapting the proof of Lemma 4.4 in [29] and using Corollary 5.9, we can show the following result.

Proposition 7.2. Suppose that assumption (2.17) holds. Then, for any $k$ in $\{0, \ldots, N\}$, there exists a unique quadruplet $\left(\boldsymbol{V}_{h}^{k}, \boldsymbol{\xi}_{\boldsymbol{V}_{h}^{k}}, \omega_{\boldsymbol{V}_{h}^{k}}, Q_{h}^{k}\right) \in \hat{\mathcal{V}}_{h}^{k} \times \hat{\mathcal{Q}}_{h}^{k}$ satisfying

$$
\begin{gathered}
2 \nu \int_{\mathcal{F}\left(\boldsymbol{\zeta}_{h}^{k}, \theta_{h}^{k}\right)} \mathbf{D}\left(\mathbf{U}^{k}-\boldsymbol{V}_{h}^{k}\right): \mathbf{D}\left(\boldsymbol{v}_{h}^{k}\right) \mathrm{d} \boldsymbol{x}-\int_{\mathcal{F}\left(\boldsymbol{\zeta}_{h}^{k}, \theta_{h}^{k}\right)}\left(\mathrm{P}^{k}-Q_{h}^{k}\right) \operatorname{div} \boldsymbol{v}_{h}^{k} \mathrm{~d} \boldsymbol{x}=0, \\
-\int_{\mathcal{F}\left(\boldsymbol{\zeta}_{h}^{k}, \theta_{h}^{k}\right)} q_{h}^{k} \operatorname{div}\left(\mathbf{U}^{k}-\boldsymbol{V}_{h}^{k}\right) \mathrm{d} \boldsymbol{x}=0,
\end{gathered}
$$

for all $\left(\boldsymbol{v}_{h}^{k}, \boldsymbol{\xi}_{\boldsymbol{v}_{h}^{k}}, \omega_{\boldsymbol{v}_{h}^{k}}, q_{h}^{k}\right)$ in $\hat{\mathcal{V}}_{h}^{k} \times \hat{\mathcal{Q}}_{h}^{k}$. Moreover, there exists a constant $C$ such that

$$
\begin{gathered}
\left\|\mathbf{U}^{k}-\boldsymbol{V}_{h}^{k}\right\|_{\mathrm{L}^{2}(\mathcal{O})^{2}} \leqslant C h\left(1+\left|\boldsymbol{\zeta}\left(t^{k}\right)-\boldsymbol{\zeta}_{h}^{k}\right|+\left|\theta\left(t^{k}\right)-\theta_{h}^{k}\right|\right)^{2}, \\
\left\|\boldsymbol{\nabla}\left(\mathbf{U}^{k}-\boldsymbol{V}_{h}^{k}\right)\right\|_{\mathrm{L}^{2}(\mathcal{O})^{4}} \leqslant C h^{1 / 2}\left(1+\left|\boldsymbol{\zeta}\left(t^{k}\right)-\boldsymbol{\zeta}_{h}^{k}\right|+\left|\theta\left(t^{k}\right)-\theta_{h}^{k}\right|\right)^{3 / 2} .
\end{gathered}
$$


Using the above proposition and equations (7.9) and (7.10), we deduce that

$$
\begin{gathered}
\int_{\mathcal{F}\left(\boldsymbol{(}_{h}^{k+1}, \theta_{h}^{k+1}\right)}\left(\frac{\mathbf{U}^{k+1}-\mathbf{U}^{k} \circ \mathcal{C}^{k}}{\delta t}\right) \cdot \boldsymbol{v}_{h}^{k+1} \mathrm{~d} \boldsymbol{x}+M \frac{\boldsymbol{\Xi}^{k+1}-\boldsymbol{\Xi}^{k}}{\delta t} \cdot \boldsymbol{\xi}_{\boldsymbol{v}_{h}}^{k}+I \frac{\Omega^{k+1}-\Omega^{k}}{\delta t} \omega_{\boldsymbol{v}_{h}}^{k} \\
+2 \nu \int_{\mathcal{F}\left(\boldsymbol{\zeta}_{h}^{k+1}, \theta_{h}^{k+1}\right)} \mathbf{D}\left(\boldsymbol{V}_{h}^{k+1}\right): \mathbf{D}\left(\boldsymbol{v}_{h}^{k+1}\right) \mathrm{d} \boldsymbol{x}-\int_{\mathcal{F}\left(\boldsymbol{\zeta}_{h}^{k+1}, \theta_{h}^{k+1}\right)} Q_{h}^{k+1} \operatorname{div} \boldsymbol{v}_{h}^{k+1} \mathrm{~d} \boldsymbol{x} \\
=\int_{\mathcal{F}\left(\boldsymbol{\zeta}_{h}^{k+1}, \theta_{h}^{k+1}\right)} \mathbf{F}^{k+1} \cdot \boldsymbol{v}_{h}^{k+1} \mathrm{~d} \boldsymbol{x}+\mathbf{F}_{M}^{k+1} \cdot \boldsymbol{\xi}_{\boldsymbol{v}_{h}^{k+1}}+\mathrm{F}_{I}^{k+1} \omega_{\boldsymbol{v}_{h}^{k+1}} \\
+\int_{\mathcal{F}\left(\boldsymbol{\zeta}_{h}^{k+1}, \theta_{h}^{k+1}\right)}\left(\mathbf{E}_{d_{t} \boldsymbol{u}}^{k}+\mathbf{E}_{\mathrm{ch}}^{k}\right) \cdot \boldsymbol{v}_{h}^{k+1} \mathrm{~d} \boldsymbol{x}+\mathbf{E}_{\boldsymbol{\xi}}^{k} \cdot \boldsymbol{\xi}_{\boldsymbol{v}_{h}^{k+1}}+\mathrm{E}_{\omega}^{k} \omega_{\boldsymbol{v}_{h}^{k+1}}, \forall\left(\boldsymbol{v}_{h}^{k+1}, \boldsymbol{\xi}_{\boldsymbol{v}_{h}^{k+1}}, \omega_{\boldsymbol{v}_{h}^{k+1}}\right) \in \hat{\boldsymbol{V}}_{h}^{k+1} \\
-\int_{\mathcal{F}\left(\boldsymbol{\zeta}_{h}^{k+1}, \theta_{h}^{k+1}\right)} q_{h}^{k+1} \operatorname{div} \boldsymbol{V}_{h}^{k+1} \mathrm{~d} \boldsymbol{x}=0, \forall q_{h}^{k+1} \in \hat{\mathcal{Q}}_{h}^{k+1}
\end{gathered}
$$

Choosing $\boldsymbol{v}_{h}^{k+1}=\boldsymbol{V}_{h}^{k+1}-\boldsymbol{u}_{h}^{k+1}$ as a test function in both (3.16) and (7.13) and subtracting the respective results, we then obtain

$$
\begin{array}{r}
\frac{1}{\delta t}\left\|\boldsymbol{V}_{h}^{k+1}-\boldsymbol{u}_{h}^{k+1}\right\|_{\mathrm{L}^{2}\left(\mathcal{F}_{h}^{k+1}\right)^{2}}^{2}+\frac{M}{\delta t}\left|\boldsymbol{\xi}_{\boldsymbol{V}_{h}^{k+1}}-\boldsymbol{\xi}_{h}^{k+1}\right|^{2}+\frac{I}{\delta t}\left|\omega_{\boldsymbol{V}_{h}^{k+1}}-\omega_{h}^{k+1}\right|^{2}+2 \nu\left\|\mathbf{D}\left(\boldsymbol{V}_{h}^{k+1}-\boldsymbol{u}_{h}^{k+1}\right)\right\|_{\mathrm{L}^{2}\left(\mathcal{F}_{h}^{k+1}\right)^{4}}^{2} \\
=\frac{1}{\delta t} \int_{\mathcal{F}_{h}^{k+1}}\left(\mathbf{U}^{k} \circ \mathcal{C}^{k}-\boldsymbol{u}_{h}^{k} \circ \mathcal{A}_{h}^{k+1, k} \circ \boldsymbol{B}_{h}^{k}\right) \cdot\left(\boldsymbol{V}_{h}^{k+1}-\boldsymbol{u}_{h}^{k+1}\right) \mathrm{d} \boldsymbol{x}+\frac{M}{\delta t}\left(\boldsymbol{\xi}_{\boldsymbol{V}_{h}^{k}}-\boldsymbol{\xi}_{h}^{k}\right) \cdot\left(\boldsymbol{\xi}_{\boldsymbol{V}_{h}^{k+1}}-\boldsymbol{\xi}_{h}^{k+1}\right) \\
+\frac{I}{\delta t}\left(\omega_{\boldsymbol{V}_{h}^{k}}-\omega_{h}^{k}\right)\left(\omega_{\boldsymbol{V}_{h}^{k+1}}-\omega_{h}^{k+1}\right)+\sum_{i=1}^{8} E_{i}
\end{array}
$$

where the terms in the indexed sum are defined as follows

$$
\begin{gathered}
E_{1}=-\int_{\mathcal{F}\left(\boldsymbol{\zeta}_{h}^{k+1}, \theta_{h}^{k+1}\right) \backslash \mathcal{F}_{h}^{k+1}}\left(\frac{\mathbf{U}^{k+1}-\mathbf{U}^{k} \circ \mathcal{C}^{k}}{\delta t}\right) \cdot\left(\boldsymbol{V}_{h}^{k+1}-\boldsymbol{u}_{h}^{k+1}\right) \mathrm{d} \boldsymbol{x}, \\
E_{2}=-\frac{1}{\delta t} \int_{\mathcal{F}_{h}^{k+1}}\left(\mathbf{U}^{k+1}-\boldsymbol{V}_{h}^{k+1}\right) \cdot\left(\boldsymbol{V}_{h}^{k+1}-\boldsymbol{u}_{h}^{k+1}\right) \mathrm{d} \boldsymbol{x}, \\
E_{3}=-\frac{M}{\delta t}\left(\boldsymbol{\Xi}^{k+1}-\boldsymbol{\xi}_{\boldsymbol{V}_{h}^{k+1}}\right) \cdot\left(\boldsymbol{\xi}_{\boldsymbol{V}_{h}^{k+1}}-\boldsymbol{\xi}_{h}^{k+1}\right), E_{4}=\frac{M}{\delta t}\left(\boldsymbol{\Xi}^{k}-\boldsymbol{\xi}_{\boldsymbol{V}_{h}^{k}}\right) \cdot\left(\boldsymbol{\xi}_{\boldsymbol{V}_{h}^{k+1}}-\boldsymbol{\xi}_{h}^{k+1}\right), \\
E_{5}=-\frac{I}{\delta t}\left(\Omega^{k+1}-\omega_{\boldsymbol{V}_{h}^{k+1}}\right) \cdot\left(\omega_{\boldsymbol{V}_{h}^{k+1}}-\omega_{h}^{k+1}\right), E_{6}=\frac{I}{\delta t}\left(\Omega^{k}-\omega_{\boldsymbol{V}_{h}^{k}}\right) \cdot\left(\omega_{\boldsymbol{V}_{h}^{k+1}}-\omega_{h}^{k+1}\right), \\
E_{7}=\int_{\mathcal{F}\left(\boldsymbol{\zeta}_{h}^{k+1}, \theta_{h}^{k+1}\right)} \mathbf{F}^{k+1} \cdot\left(\boldsymbol{V}_{h}^{k+1}-\boldsymbol{u}_{h}^{k+1}\right) \mathrm{d} \boldsymbol{x}-\int_{\mathcal{F}_{h}^{k+1}} \boldsymbol{f}_{h}^{k+1} \cdot\left(\boldsymbol{V}_{h}^{k+1}-\boldsymbol{u}_{h}^{k+1}\right) \mathrm{d} \boldsymbol{x}, \\
E_{8}=\int_{\mathcal{F}\left(\boldsymbol{\zeta}_{h}^{k+1}, \theta_{h}^{k+1}\right)}\left(\mathbf{E}_{d_{t} \boldsymbol{u}}^{k}+\mathbf{E}_{\mathrm{ch}}^{k}\right) \cdot\left(\boldsymbol{V}_{h}^{k+1}-\boldsymbol{u}_{h}^{k+1}\right) \cdot\left(\boldsymbol{\xi}_{\boldsymbol{V}_{h}^{k+1}}-\boldsymbol{\xi}_{h}^{k+1}\right)+\left(\mathrm{F}_{I}^{k+1}-f_{h, I}^{k+1}\right)\left(\omega_{\boldsymbol{V}_{h}^{k+1}}-\omega_{h}^{k+1}\right), \\
\mathbf{E}_{\boldsymbol{\xi}}^{k} \cdot\left(\boldsymbol{\xi}_{\boldsymbol{V}_{h}^{k+1}}-\boldsymbol{\xi}_{h}^{k+1}\right)+\mathrm{E}_{\omega}^{k}\left(\omega_{\boldsymbol{V}_{h}^{k+1}}-\omega_{h}^{k+1}\right) .
\end{gathered}
$$

We estimate $\left|E_{1}\right|$ by first recalling that

$\frac{\mathbf{U}^{k+1}-\mathbf{U}^{k} \circ \mathcal{C}^{k}}{\delta t}=\mathbf{E}_{d_{t} \boldsymbol{u}}^{k}+\left(J_{\mathbf{Y}^{k+1}} \circ \mathbf{X}^{k+1}\right) \frac{\mathrm{d}}{\mathrm{d} t}[\boldsymbol{u} \circ \mathcal{C}]\left(\mathbf{X}^{k+1}, t^{k+1}\right)+\frac{\left(\left(J_{\mathbf{Y}^{k+1}} \circ \mathbf{X}^{k+1}\right)\left(J_{\mathbf{X}^{k}} \circ \mathcal{C}^{k}\right)-\mathbf{I d}\right)\left(\mathbf{U}^{k} \circ \mathcal{C}^{k}\right)}{\delta t}$. 
Lemmata 4.1, 4.3 and 6.4, property (3.2), regularity hypotheses (3.18) and bound (7.12) are therefore used to find that

$$
\begin{aligned}
\left|E_{1}\right| \leqslant & C\left(1+\left|\boldsymbol{\zeta}\left(t^{k+1}\right)-\boldsymbol{\zeta}_{h}^{k+1}\right|+\left|\theta\left(t^{k+1}\right)-\theta_{h}^{k+1}\right|\right) \\
& \times\left(1+\left|\boldsymbol{\zeta}\left(t^{k}\right)-\boldsymbol{\zeta}_{h}^{k}\right|+\left|\theta\left(t^{k}\right)-\theta_{h}^{k}\right|+\left|\boldsymbol{\xi}\left(t^{k}\right)-\boldsymbol{\xi}_{h}^{k}\right|+\left|\omega\left(t^{k}\right)-\omega_{h}^{k}\right|\right)^{2} \\
& \times\left(\left|\boldsymbol{\zeta}\left(t^{k}\right)-\boldsymbol{\zeta}_{h}^{k}\right|+\left|\theta\left(t^{k}\right)-\theta_{h}^{k}\right|+\left|\boldsymbol{\xi}\left(t^{k}\right)-\boldsymbol{\xi}_{h}^{k}\right|+\left|\omega\left(t^{k}\right)-\omega_{h}^{k}\right|+\delta t+h^{1 / 2}\right)\left\|\boldsymbol{V}_{h}^{k+1}-\boldsymbol{u}_{h}^{k+1}\right\|_{L^{2}(\mathcal{O})^{2}}
\end{aligned}
$$

The terms $\left|E_{i}\right|, i=2, \ldots, 6$, can be bounded using Proposition 7.2 , which gives

$$
\sum_{i=2}^{6}\left|E_{i}\right| \leqslant C \frac{h}{\delta t}\left(1+\left|\boldsymbol{\zeta}\left(t^{k+1}\right)-\boldsymbol{\zeta}_{h}^{k+1}\right|+\left|\theta\left(t^{k+1}\right)-\theta_{h}^{k+1}\right|\right)^{2}\left\|\boldsymbol{V}_{h}^{k+1}-\boldsymbol{u}_{h}^{k+1}\right\|_{L^{2}(\mathcal{O})^{2}}
$$

In order to deal with $\left|E_{7}\right|$, let us first remark that, since $\mathcal{S}\left(\boldsymbol{\zeta}_{h}^{k+1}, \theta_{h}^{k+1}\right) \subset \mathcal{S}_{h}^{k+1}$ by construction, we have, from definitions (2.4), the respective definitions of $\mathbf{F}^{k+1}, \mathbf{F}_{M}^{k+1}$ and $\mathrm{F}_{I}^{k+1}$, and property (4.4) of the change of variables $\mathbf{X}^{k+1}$,

$$
\begin{gathered}
\int_{\mathcal{F}\left(\boldsymbol{\zeta}_{h}^{k+1}, \theta_{h}^{k+1}\right)} \mathbf{F}^{k+1} \cdot \boldsymbol{v}_{h}^{k+1} \mathrm{~d} \boldsymbol{x}-\int_{\mathcal{F}_{h}^{k+1}} \boldsymbol{f}_{h}^{k+1} \cdot \boldsymbol{v}_{h}^{k+1} \mathrm{~d} \boldsymbol{x}+\left(\mathbf{F}_{M}^{k+1}-\boldsymbol{f}_{h, M}^{k+1}\right) \cdot \boldsymbol{\xi}_{\boldsymbol{v}_{h}^{k+1}}+\left(\mathrm{F}_{I}^{k+1}-f_{h, I}^{k+1}\right) \omega_{\boldsymbol{v}_{h}^{k+1}} \\
=\int_{\mathcal{F}\left(\boldsymbol{\zeta}_{h}^{k+1}, \theta_{h}^{k+1}\right)} \mathbf{F}^{k+1} \cdot \boldsymbol{v}_{h}^{k+1} \mathrm{~d} \boldsymbol{x}+\int_{\mathcal{S}\left(\boldsymbol{\zeta}_{h}^{k+1}, \theta_{h}^{k+1}\right)} \rho_{s} \mathbf{F}^{k+1} \cdot \boldsymbol{v}_{h}^{k+1} \mathrm{~d} \boldsymbol{x} \\
\quad-\int_{\mathcal{F}_{h}^{k+1}} \boldsymbol{f}_{h}^{k+1} \cdot \boldsymbol{v}_{h}^{k+1} \mathrm{~d} \boldsymbol{x}-\int_{\mathcal{S}_{h}^{k+1}} \rho_{s} \boldsymbol{f}_{h}^{k+1} \cdot \boldsymbol{v}_{h}^{k+1} \mathrm{~d} \boldsymbol{x} .
\end{gathered}
$$

The field $\boldsymbol{f}_{h}^{k+1}$ being the projection of $\boldsymbol{f}\left(\cdot, t^{k+1}\right)$ on $\left(\widetilde{\mathcal{P}}_{h}^{k+1}\right)^{2}$, it satisfies, owing to Corollary 5.7,

$$
\left\|\boldsymbol{f}\left(\cdot, t^{k+1}\right)-\boldsymbol{f}_{h}^{k+1}\right\|_{L^{2}(\mathcal{O})^{2}} \leqslant C h\left(1+\left|\boldsymbol{\zeta}\left(t^{k+1}\right)-\boldsymbol{\zeta}_{h}^{k+1}\right|+\left|\theta\left(t^{k+1}\right)-\theta_{h}^{k+1}\right|\right) .
$$

Note that the positive constant $C$ in the above inequality can indeed be chosen in such a way that it does not depend on $k$, as it is assumed that (2.17) holds for some constant $c_{0}$ small enough. It then follows from the above estimate, Lemma 4.1, property (3.2) and assumption (3.19) that

$$
\left|E_{7}\right| \leqslant C\left(\left|\boldsymbol{\zeta}\left(t^{k+1}\right)-\boldsymbol{\zeta}_{h}^{k+1}\right|+\left|\theta\left(t^{k+1}\right)-\theta_{h}^{k+1}\right|+h^{1 / 2}\right)\left\|\boldsymbol{V}_{h}^{k+1}-\boldsymbol{u}_{h}^{k+1}\right\|_{L^{2}(\mathcal{O})^{2}}
$$

Finally, we infer from Lemmata 4.1 and 7.1 and estimate (7.12) that

$$
\begin{aligned}
\left|E_{8}\right| \leqslant C\left[\left(1+\mid \boldsymbol{\zeta}\left(t^{k+1}\right)\right.\right. & \left.-\boldsymbol{\zeta}_{h}^{k+1}|+| \theta\left(t^{k+1}\right)-\theta_{h}^{k+1} \mid\right) \\
& \times\left(1+\left|\boldsymbol{\zeta}\left(t^{k}\right)-\boldsymbol{\zeta}_{h}^{k}\right|+\left|\theta\left(t^{k}\right)-\theta_{h}^{k}\right|+\left|\boldsymbol{\xi}\left(t^{k}\right)-\boldsymbol{\xi}_{h}^{k}\right|+\left|\omega\left(t^{k}\right)-\omega_{h}^{k}\right|\right)^{2} \\
& \times\left(\left|\boldsymbol{\zeta}\left(t^{k}\right)-\boldsymbol{\zeta}_{h}^{k}\right|+\left|\theta\left(t^{k}\right)-\theta_{h}^{k}\right|+\left|\boldsymbol{\xi}\left(t^{k}\right)-\boldsymbol{\xi}_{h}^{k}\right|+\left|\omega\left(t^{k}\right)-\omega_{h}^{k}\right|+\delta t\right) \\
& \left.\quad+\left(\left|\boldsymbol{\zeta}\left(t^{k+1}\right)-\boldsymbol{\zeta}_{h}^{k+1}\right|+\left|\theta\left(t^{k+1}\right)-\theta_{h}^{k+1}\right|\right)\right]\left\|\boldsymbol{V}_{h}^{k+1}-\boldsymbol{u}_{h}^{k+1}\right\|_{L^{2}(\mathcal{O})^{2}}
\end{aligned}
$$


Next, the first term in the right-hand side of (7.15) can be decomposed as follows

$$
\begin{array}{r}
\int_{\mathcal{F}_{h}^{k+1}}\left(\mathbf{U}^{k} \circ \mathcal{C}^{k}-\boldsymbol{u}_{h}^{k} \circ \mathcal{A}_{h}^{k+1, k} \circ \mathcal{B}_{h}^{k}\right) \cdot\left(\boldsymbol{V}_{h}^{k+1}-\boldsymbol{u}_{h}^{k+1}\right) \mathrm{d} \boldsymbol{x}=\int_{\mathcal{F}_{h}^{k+1}}\left(\left(\mathbf{U}^{k}-\boldsymbol{u}_{h}^{k}\right) \circ \mathcal{C}^{k}\right) \cdot\left(\boldsymbol{V}_{h}^{k+1}-\boldsymbol{u}_{h}^{k+1}\right) \mathrm{d} \boldsymbol{x} \\
-\int_{\mathcal{F}_{h}^{k+1}}\left(\left(\mathbf{U}^{k}-\boldsymbol{u}_{h}^{k}\right) \circ \mathcal{C}^{k}-\left(\mathbf{U}^{k}-\boldsymbol{u}_{h}^{k}\right) \circ \mathcal{A}_{h}^{k+1, k} \circ \mathcal{B}_{h}^{k}\right) \cdot\left(\boldsymbol{V}_{h}^{k+1}-\boldsymbol{u}_{h}^{k+1}\right) \mathrm{d} \boldsymbol{x} \\
\quad+\int_{\mathcal{F}_{h}^{k+1}}\left(\mathbf{U}^{k} \circ \mathcal{C}^{k}-\mathbf{U}^{k} \circ \mathcal{A}_{h}^{k+1, k} \circ \mathcal{B}_{h}^{k}\right) \cdot\left(\boldsymbol{V}_{h}^{k+1}-\boldsymbol{u}_{h}^{k+1}\right) \mathrm{d} \boldsymbol{x} .
\end{array}
$$

Denoting respectively by $I_{1}, I_{2}$ and $I_{3}$ the three integrals in the right-hand side above, we first check that

$$
\begin{aligned}
\left|I_{1}\right| \leqslant \frac{1}{2}\left(\left\|\boldsymbol{V}_{h}^{k+1}-\boldsymbol{u}_{h}^{k+1}\right\|_{\mathrm{L}^{2}\left(\mathcal{F}_{h}^{k+1}\right)^{2}}^{2}+\|\right. & \left.\boldsymbol{V}_{h}^{k}-\boldsymbol{u}_{h}^{k} \|_{\mathrm{L}^{2}\left(\mathcal{F}_{h}^{k}\right)^{2}}^{2}\right) \\
& +C h\left(1+\left|\boldsymbol{\zeta}\left(t^{k}\right)-\boldsymbol{\zeta}_{h}^{k}\right|+\left|\theta\left(t^{k}\right)-\theta_{h}^{k}\right|\right)^{2}\left\|\boldsymbol{V}_{h}^{k+1}-\boldsymbol{u}_{h}^{k+1}\right\|_{\mathrm{L}^{2}(\mathcal{O})^{2}}
\end{aligned}
$$

Then, arguing as in [1], we have

$$
\left|I_{2}\right| \leqslant\left\|\left(\mathbf{U}^{k}-\boldsymbol{u}_{h}^{k}\right) \circ \mathcal{C}^{k}-\left(\mathbf{U}^{k}-\boldsymbol{u}_{h}^{k}\right) \circ \mathcal{A}_{h}^{k+1, k} \circ \mathcal{B}_{h}^{k}\right\|_{L^{1}\left(\mathcal{F}_{h}^{k+1}\right)^{2}}\left\|\boldsymbol{V}_{h}^{k+1}-\boldsymbol{u}_{h}^{k+1}\right\|_{L^{\infty}\left(\mathcal{F}_{h}^{k+1}\right)^{2}},
$$

which yields, using an inverse inequality (see for instance [3]), Lemmata 5.3 and 6.3, and Corollary 5.9,

$$
\begin{aligned}
\left|I_{2}\right| \leqslant C\left(|\log h|+\left|\boldsymbol{\zeta}\left(t^{k+1}\right)-\boldsymbol{\zeta}_{h}^{k+1}\right|+\left|\theta\left(t^{k+1}\right)-\theta_{h}^{k+1}\right|\right)^{1 / 2} & \left\|\boldsymbol{\nabla}\left(\mathbf{U}^{k}-\boldsymbol{u}_{h}^{k}\right)\right\|_{\mathrm{L}^{2}(\mathcal{O})^{4}} \\
& \times\left\|\mathcal{C}^{k}-\mathcal{A}_{h}^{k+1, k} \circ \mathcal{B}_{h}^{k}\right\|_{\mathrm{L}^{2}(\mathcal{O})^{2}}\left\|\boldsymbol{V}_{h}^{k+1}-\boldsymbol{u}_{h}^{k+1}\right\|_{\mathrm{H}^{1}\left(\mathcal{F}_{h}^{k+1}\right)^{2}} .
\end{aligned}
$$

Then, by the Poincaré and Korn inequalities, Theorem 6.1 and Proposition 7.2, it follows that

$$
\begin{aligned}
\left|I_{2}\right| \leqslant C(\delta t)(|\log h| & \left.+\left|\boldsymbol{\zeta}\left(t^{k+1}\right)-\boldsymbol{\zeta}_{h}^{k+1}\right|+\left|\theta\left(t^{k+1}\right)-\theta_{h}^{k+1}\right|\right)^{1 / 2} \\
& \times\left(\left\|\mathbf{D}\left(\boldsymbol{V}_{h}^{k}-\boldsymbol{u}_{h}^{k}\right)\right\|_{\mathrm{L}^{2}\left(\mathcal{F}_{h}^{k}\right)^{4}}+h^{1 / 2}\left(1+\left|\boldsymbol{\zeta}\left(t^{k}\right)-\boldsymbol{\zeta}_{h}^{k}\right|+\left|\theta\left(t^{k}\right)-\theta_{h}^{k}\right|\right)^{3 / 2}\right) \\
\times\left(\| \mathbf{U}^{k}-\right. & \left.\boldsymbol{u}_{h}^{k} \|_{\mathrm{L}^{2}(\mathcal{O})^{2}}+\left|\boldsymbol{\zeta}\left(t^{k}\right)-\boldsymbol{\zeta}_{h}^{k}\right|+\left|\theta\left(t^{k}\right)-\theta_{h}^{k}\right|+\delta t+h^{1 / 2}\right)\left\|\mathbf{D}\left(\boldsymbol{V}_{h}^{k+1}-\boldsymbol{u}_{h}^{k+1}\right)\right\|_{\mathrm{L}^{2}\left(\mathcal{F}_{h}^{k+1}\right)^{4}}
\end{aligned}
$$

The last term can be treated as follows

$$
\left|I_{3}\right| \leqslant C\left(1+\left|\boldsymbol{\zeta}\left(t^{k}\right)-\boldsymbol{\zeta}_{h}^{k}\right|+\left|\theta\left(t^{k}\right)-\theta_{h}^{k}\right|\right)\left\|\mathcal{C}^{k}-\mathcal{A}_{h}^{k+1, k} \circ \mathcal{B}_{h}^{k}\right\|_{L^{2}\left(\mathcal{F}_{h}^{k+1}\right)^{2}}\left\|\boldsymbol{V}_{h}^{k+1}-\boldsymbol{u}_{h}^{k+1}\right\|_{L^{2}\left(\mathcal{F}_{h}^{k+1}\right)^{2}},
$$

hence

$$
\begin{aligned}
\left|I_{3}\right| \leqslant C(\delta t)\left(1+\left|\boldsymbol{\zeta}\left(t^{k}\right)-\boldsymbol{\zeta}_{h}^{k}\right|+\mid \theta\left(t^{k}\right)\right. & \left.-\theta_{h}^{k} \mid\right)\left\|\boldsymbol{V}_{h}^{k+1}-\boldsymbol{u}_{h}^{k+1}\right\|_{L^{2}\left(\mathcal{F}_{h}^{k+1}\right)^{2}} \\
& \times\left(\left\|\mathbf{U}^{k}-\boldsymbol{u}_{h}^{k}\right\|_{L^{2}(\mathcal{O})^{2}}+\left|\boldsymbol{\zeta}\left(t^{k}\right)-\boldsymbol{\zeta}_{h}^{k}\right|+\left|\theta\left(t^{k}\right)-\theta_{h}^{k}\right|+\delta t+h^{1 / 2}\right) .
\end{aligned}
$$


Substituting bounds (7.16) to (7.23) into identity (7.15) and using the triangle inequality with the first estimate in Proposition 7.2, we obtain

$$
\begin{gathered}
\left\|\boldsymbol{V}_{h}^{k+1}-\boldsymbol{u}_{h}^{k+1}\right\|_{\mathrm{L}^{2}\left(\mathcal{F}_{h}^{k+1}\right)^{2}}^{2}+M\left|\boldsymbol{\xi}_{\boldsymbol{V}_{h}^{k+1}}-\boldsymbol{\xi}_{h}^{k+1}\right|^{2}+I\left|\omega_{\boldsymbol{V}_{h}^{k+1}}-\omega_{h}^{k+1}\right|^{2}+4 \nu(\delta t)\left\|\mathbf{D}\left(\boldsymbol{V}_{h}^{k+1}-\boldsymbol{u}_{h}^{k+1}\right)\right\|_{\mathrm{L}^{2}\left(\mathcal{F}_{h}^{k+1}\right)^{4}}^{2} \\
\leqslant\left\|\boldsymbol{V}_{h}^{k}-\boldsymbol{u}_{h}^{k}\right\|_{\mathrm{L}^{2}\left(\mathcal{F}_{h}^{k}\right)^{2}}+M\left|\boldsymbol{\xi}_{\boldsymbol{V}_{h}^{k}}-\boldsymbol{\xi}_{h}^{k}\right|^{2}+I\left|\omega_{\boldsymbol{V}_{h}^{k}}-\omega_{h}^{k}\right|^{2}+C\left(1+\left|\boldsymbol{\zeta}\left(t^{k+1}\right)-\boldsymbol{\zeta}_{h}^{k+1}\right|+\left|\theta\left(t^{k+1}\right)-\theta_{h}^{k+1}\right|\right)^{3} \\
\times\left((\delta t)\left(\left|\boldsymbol{\zeta}\left(t^{k+1}\right)-\boldsymbol{\zeta}_{h}^{k+1}\right|+\left|\theta\left(t^{k+1}\right)-\theta_{h}^{k+1}\right|+\left\|\boldsymbol{V}_{h}^{k}-\boldsymbol{u}_{h}^{k}\right\|_{\mathrm{L}^{2}(\mathcal{O})^{2}}+\left|\boldsymbol{\zeta}\left(t^{k}\right)-\boldsymbol{\zeta}_{h}^{k}\right|+\left|\theta\left(t^{k}\right)-\theta_{h}^{k}\right|\right)+(\delta t)^{2}+h\right) \\
\times\left(1+\left\|\boldsymbol{V}_{h}^{k}-\boldsymbol{u}_{h}^{k}\right\|_{\mathrm{L}^{2}(\mathcal{O})^{2}}+\left|\boldsymbol{\zeta}\left(t^{k}\right)-\boldsymbol{\zeta}_{h}^{k}\right|+\left|\theta\left(t^{k}\right)-\theta_{h}^{k}\right|\right)^{2}\left\|\boldsymbol{V}_{h}^{k+1}-\boldsymbol{u}_{h}^{k+1}\right\|_{\mathrm{L}^{2}(\mathcal{O})^{2}} \\
+C(\delta t)\left(1+\left|\boldsymbol{\zeta}\left(t^{k}\right)-\boldsymbol{\zeta}_{h}^{k}\right|+\left|\theta\left(t^{k}\right)-\theta_{h}^{k}\right|\right)^{4}\left(|\log h|+\left|\boldsymbol{\zeta}\left(t^{k+1}\right)-\boldsymbol{\zeta}_{h}^{k+1}\right|+\left|\theta\left(t^{k+1}\right)-\theta_{h}^{k+1}\right|\right)^{1 / 2} \\
\times\left(\left\|\boldsymbol{V}_{h}^{k}-\boldsymbol{u}_{h}^{k}\right\|_{\mathrm{L}^{2}(\mathcal{O})^{2}}+\left|\boldsymbol{\zeta}\left(t^{k}\right)-\boldsymbol{\zeta}_{h}^{k}\right|+\left|\theta\left(t^{k}\right)-\theta_{h}^{k}\right|+\delta t+h^{1 / 2}\right) \\
\times\left(\left\|\mathbf{D}\left(\boldsymbol{V}_{h}^{k}-\boldsymbol{u}_{h}^{k}\right)\right\|_{\mathrm{L}^{2}\left(\mathcal{F}_{h}^{k}\right)^{4}}+h^{1 / 2}\right)\left\|\mathbf{D}\left(\boldsymbol{V}_{h}^{k+1}-\boldsymbol{u}_{h}^{k+1}\right)\right\|_{\mathrm{L}^{2}\left(\mathcal{F}_{h}^{k+1}\right)^{4}} .
\end{gathered}
$$

Denoting, for all $k$ in $\{0, \ldots, N\}$,

$$
\mathcal{N}^{k}=\left\|\boldsymbol{V}_{h}^{k}-\boldsymbol{u}_{h}^{k}\right\|_{L^{2}\left(\mathcal{F}_{h}^{k}\right)^{2}}^{2}+M\left|\boldsymbol{\xi}_{V_{h}^{k}}-\boldsymbol{\xi}_{h}^{k}\right|^{2}+I\left|\omega_{V_{h}^{k}}-\omega_{h}^{k}\right|^{2},
$$

we infer on the one hand, using the Taylor-Lagrange inequality and regularity assumptions (3.18), that

$$
\begin{gathered}
\left|\boldsymbol{\zeta}\left(t^{k+1}\right)-\boldsymbol{\zeta}_{h}^{k+1}\right|+\left|\theta\left(t^{k+1}\right)-\theta_{h}^{k+1}\right| \leqslant\left|\boldsymbol{\zeta}\left(t^{k}\right)-\boldsymbol{\zeta}_{h}^{k}\right|+\left|\theta\left(t^{k}\right)-\theta_{h}^{k}\right|+C(\delta t)\left(\sqrt{\mathcal{N}^{k}}+(\delta t)\right) \\
\left|\boldsymbol{\zeta}\left(t^{k+1}\right)-\boldsymbol{\zeta}_{h}^{k+1}\right|^{2}+\left|\theta\left(t^{k+1}\right)-\theta_{h}^{k+1}\right|^{2} \leqslant(1+C(\delta t))\left(\left|\boldsymbol{\zeta}\left(t^{k}\right)-\boldsymbol{\zeta}_{h}^{k}\right|^{2}+\left|\theta\left(t^{k}\right)-\theta_{h}^{k}\right|^{2}\right)+C(\delta t)\left(\mathcal{N}^{k}+(\delta t)^{2}\right),
\end{gathered}
$$

and on the other hand, from (7.24), (7.25), the use of condition (3.20), Young's inequality, hypotheses (3.18) and some estimates, that

$$
\begin{aligned}
& \mathcal{N}^{k+1}+ 4 \nu(\delta t)\left\|\mathbf{D}\left(\boldsymbol{V}_{h}^{k+1}-\boldsymbol{u}_{h}^{k+1}\right)\right\|_{\mathrm{L}^{2}\left(\mathcal{F}_{h}^{k+1}\right)^{4}}^{2} \leqslant \mathcal{N}^{k}+C(\delta t)\left(1+\left|\boldsymbol{\zeta}\left(t^{k}\right)-\boldsymbol{\zeta}_{h}^{k}\right|+\left|\theta\left(t^{k}\right)-\theta_{h}^{k}\right|+\sqrt{\mathcal{N}^{k}}\right)^{6} \\
& \times\left(\left|\boldsymbol{\zeta}\left(t^{k}\right)-\boldsymbol{\zeta}_{h}^{k}\right|+\left|\theta\left(t^{k}\right)-\theta_{h}^{k}\right|+\sqrt{\mathcal{N}^{k}}+\delta t\right) \sqrt{\mathcal{N}^{k+1}}+C(\delta t)\left(1+\left|\boldsymbol{\zeta}\left(t^{k}\right)-\boldsymbol{\zeta}_{h}^{k}\right|+\left|\theta\left(t^{k}\right)-\theta_{h}^{k}\right|\right)^{4} \\
& \times\left(|\log \delta t|+\left|\boldsymbol{\zeta}\left(t^{k}\right)-\boldsymbol{\zeta}_{h}^{k}\right|+\left|\theta\left(t^{k}\right)-\theta_{h}^{k}\right|+\sqrt{\mathcal{N}^{k}}\right)^{1 / 2}\left(\left|\boldsymbol{\zeta}\left(t^{k}\right)-\boldsymbol{\zeta}_{h}^{k}\right|+\left|\theta\left(t^{k}\right)-\theta_{h}^{k}\right|+\sqrt{\mathcal{N}^{k}}+\delta t\right) \\
& \times\left(\left\|\mathbf{D}\left(\boldsymbol{V}_{h}^{k}-\boldsymbol{u}_{h}^{k}\right)\right\|_{\mathrm{L}^{2}\left(\mathcal{F}_{h}^{k}\right)^{4}}^{2}+\left\|\mathbf{D}\left(\boldsymbol{V}_{h}^{k+1}-\boldsymbol{u}_{h}^{k+1}\right)\right\|_{\mathrm{L}^{2}\left(\mathcal{F}_{h}^{k+1}\right)^{4}}^{2}+(\delta t)^{2}\right) .
\end{aligned}
$$

We are finally in a position to demonstrate the main result of the paper.

Proof of Theorem 3.5. We proceed by induction on $k$ to show that bounds (6.1) and (6.2) and the following induction relation

$$
\mathcal{N}^{k}+2 \nu(\delta t)\left\|\mathbf{D}\left(\boldsymbol{V}_{h}^{k}-\boldsymbol{u}_{h}^{k}\right)\right\|_{\mathrm{L}^{2}\left(\mathcal{F}_{h}^{k}\right)^{4}}^{2}+\left|\boldsymbol{\zeta}\left(t^{k}\right)-\boldsymbol{\zeta}_{h}^{k}\right|^{2}+\left|\theta\left(t^{k}\right)-\theta_{h}^{k}\right|^{2} \leqslant C_{k}(\delta t)^{2},
$$

hold for all $k$ in $\{0, \ldots, N\}$, with

$$
C_{k}=(1+642 C(\delta t))^{k} C_{0}+(4 \nu+130) \frac{(1+642 C(\delta t))^{k}-1}{642 C},
$$


where $C$ is the maximum of the fixed generic constants appearing in (7.26) and (7.27). Notice there exists a positive constant $C_{\infty}$, independent of $\delta t$ and $h$, such that, for any $k$ in $\{0, \ldots, N\}$,

$$
C_{k} \leqslant C_{\infty}
$$

The first step is to show that these statements are true when $k=0$. From the respective definitions of $\boldsymbol{u}_{h}^{0}$, $\boldsymbol{\zeta}_{h}^{0}, \theta_{h}^{0}, \boldsymbol{\xi}_{h}^{0}$ and $\omega_{h}^{0}$ in Subsection 3.1.1 and assumption (2.1) on the regularity of the initial datum $\boldsymbol{u}^{(0)}$, we have

$$
\mathcal{N}^{0}+2 \nu(\delta t)\left\|\mathbf{D}\left(\boldsymbol{V}_{h}^{0}-\boldsymbol{u}_{h}^{0}\right)\right\|_{\mathrm{L}^{2}\left(\mathcal{F}_{h}^{0}\right)^{4}}^{2} \leqslant C_{0}(\delta t)^{2},
$$

for some positive constant $C_{0}$. It remains only to prove that (6.1) and (6.2) are satisfied, that is

$$
(\delta t)\|\overline{\boldsymbol{u}}-\overline{\boldsymbol{w}}\|_{\mathrm{L}^{\infty}\left(0, t^{1} ; \mathrm{W}^{1, \infty}(\mathcal{O})\right)^{2}} \leqslant \varepsilon \text { and }(\delta t)\left\|\overline{\boldsymbol{u}}_{\boldsymbol{h}}^{\mathbf{0}}-\overline{\boldsymbol{w}}_{\boldsymbol{h}}^{\mathbf{0}}\right\|_{\mathrm{W}^{1, \infty}(\mathcal{O})^{2}} \leqslant \varepsilon,
$$

where, for all $t$ in $\left[0, t^{1}\right]$ and $\boldsymbol{x}$ in $\mathcal{F}\left(\boldsymbol{\zeta}\left(t^{1}\right), \theta\left(t^{1}\right)\right)$, the functions $\overline{\boldsymbol{u}}$ and $\overline{\boldsymbol{w}}$ are defined by taking $s$ equal to $t^{1}$ in (2.21) and $\overline{\boldsymbol{u}}_{h}^{0}$ and $\overline{\boldsymbol{w}}_{h}^{0}$ are given by (3.15). The first bound being only a condition on the time step, we check the second one by using an inverse inequality

$$
(\delta t)\left\|\overline{\boldsymbol{u}}_{\boldsymbol{h}}^{0}-\overline{\boldsymbol{w}}_{\boldsymbol{h}}^{0}\right\|_{\mathrm{W}^{1, \infty}(\mathcal{O})^{2}} \leqslant(\delta t)|\log h|^{1 / 2}\left\|\overline{\boldsymbol{u}}_{\boldsymbol{h}}^{0}-\overline{\boldsymbol{w}}_{\boldsymbol{h}}^{0}\right\|_{\mathrm{H}^{1}(\mathcal{O})^{2}} .
$$

Then, from assumption (2.17) and the properties of the mapping $\mathcal{A}_{h}^{0}$, we get that

$$
(\delta t)\left\|\overline{\boldsymbol{u}}_{\boldsymbol{h}}^{0}-\overline{\boldsymbol{w}}_{\boldsymbol{h}}^{0}\right\|_{\mathrm{W}^{1, \infty}(\mathcal{O})^{2}} \leqslant C(\delta t)|\log h|^{1 / 2} .
$$

The above relation and condition (3.20) thus imply the result for $k=0$.

Now, assuming that the relations (6.1), (6.2) and (7.28) hold for some $k \geqslant 0$, we will show they are also true for $k+1$. For $\delta t$ small enough, relation (6.1) is simply a consequence of Lemma 4.1 and results (5.2) to (5.4). Next, assuming that $\delta t$ is such that

$$
\sqrt{3 C_{\infty}}(\delta t) \leqslant 1 \text { and } 16 C(\delta t)(|\log \delta t|+1)^{1 / 2}\left(\sqrt{3 C_{\infty}}+1\right)<2 \nu,
$$

we infer from (7.27) that

$$
\begin{aligned}
\mathcal{N}^{k+1}+2 \nu(\delta t)\left\|\mathbf{D}\left(\boldsymbol{V}_{h}^{k+1}-\boldsymbol{u}_{h}^{k+1}\right)\right\|_{\mathrm{L}^{2}\left(\mathcal{F}_{h}^{k+1}\right)^{3}}^{2} & \leqslant \mathcal{N}^{k}+2 \nu(\delta t)\left\|\mathbf{D}\left(\boldsymbol{V}_{h}^{k}-\boldsymbol{u}_{h}^{k}\right)\right\|_{\mathrm{L}^{2}\left(\mathcal{F}_{h}^{k}\right)^{3}}^{2}+128 C(\delta t) \mathcal{N}^{k+1} \\
& +192 C(\delta t)\left(\mathcal{N}^{k}+\left|\boldsymbol{\zeta}\left(t^{k}\right)-\boldsymbol{\zeta}_{h}^{k}\right|^{2}+\left|\theta\left(t^{k}\right)-\theta_{h}^{k}\right|^{2}\right)+(64 C+2 \nu)(\delta t)^{3} .
\end{aligned}
$$

Using (7.26) and the latter inequality, we find that

$$
\begin{aligned}
&\left(\mathcal{N}^{k+1}+\right.\left.2 \nu(\delta t)\left\|\mathbf{D}\left(\boldsymbol{V}_{h}^{k+1}-\boldsymbol{u}_{h}^{k+1}\right)\right\|_{\mathrm{L}^{2}\left(\mathcal{F}_{h}^{k+1}\right)^{4}}^{2}+\left|\boldsymbol{\zeta}\left(t^{k+1}\right)-\boldsymbol{\zeta}_{h}^{k+1}\right|^{2}+\left|\theta\left(t^{k+1}\right)-\theta_{h}^{k+1}\right|^{2}\right) \leqslant \\
&(1+642 C \delta t)\left(\mathcal{N}^{k}+2 \nu(\delta t)\left\|\mathbf{D}\left(\boldsymbol{V}_{h}^{k}-\boldsymbol{u}_{h}^{k}\right)\right\|_{\mathrm{L}^{2}\left(\mathcal{F}_{h}^{k}\right)^{4}}^{2}+\left|\boldsymbol{\zeta}\left(t^{k}\right)-\boldsymbol{\zeta}_{h}^{k}\right|^{2}+\left|\theta\left(t^{k}\right)-\theta_{h}^{k}\right|^{2}\right)+(130+4 \nu)(\delta t)^{3},
\end{aligned}
$$

which clearly implies (7.28) and (7.29). It remains to prove that (6.2) holds for $k+1$. We have

$$
\begin{aligned}
(\delta t)\left\|\overline{\boldsymbol{u}}_{\boldsymbol{h}}^{k+1}-\overline{\boldsymbol{w}}_{\boldsymbol{h}}^{k+\mathbf{1}}\right\|_{\mathrm{W}^{1, \infty}(\mathcal{O})^{2}} \leqslant(\delta t)\left(\left\|\overline{\boldsymbol{u}}_{\boldsymbol{h}}^{k+1}-\boldsymbol{V}_{h}^{k+1}\right\|_{\mathrm{W}^{1, \infty}(\mathcal{O})^{2}}+\left\|\overline{\boldsymbol{w}}_{\boldsymbol{h}}^{\boldsymbol{k}+\boldsymbol{1}}-\boldsymbol{W}_{h}^{k+1}\right\|_{\mathrm{W}^{1, \infty}(\mathcal{O})^{2}}\right. & \left.+\left\|\boldsymbol{V}_{h}^{k+1}\right\|_{\mathrm{W}^{1, \infty}(\mathcal{O})^{2}}+\left\|\boldsymbol{W}_{h}^{k+1}\right\|_{\mathrm{W}^{1, \infty}(\mathcal{O})^{2}}\right), \\
&
\end{aligned}
$$


which yields, using an inverse inequality and some estimates,

$$
(\delta t)\left\|\overline{\boldsymbol{u}}_{\boldsymbol{h}}^{\boldsymbol{k}+\mathbf{1}}-\overline{\boldsymbol{w}}_{\boldsymbol{h}}^{k+\mathbf{1}}\right\|_{\mathrm{W}^{1, \infty}(\mathcal{O})^{2}} \leqslant C(\delta t)\left(|\log \delta t|^{1 / 2}+\delta t\right) .
$$

Taking the time step small enough thus gives the desired result.

The two estimates stated in the theorem finally derive from (7.28). Indeed, combining this bound with (7.30) and the results of Lemma 4.1 and Proposition 7.2, we find that

$$
\left\|\boldsymbol{u}\left(\cdot, t^{k}\right)-\boldsymbol{u}_{h}^{k}\right\|_{\mathrm{L}^{2}\left(\mathcal{O}^{2}\right)} \leqslant C(\delta t),
$$

and reach the conclusion by using the error bounds obtained above, assumptions (3.18) and Lemma 5.1.

\section{REFERENCES}

[1] Y. Achdou and J.-L. Guermond, Convergence analysis of a finite element projection/Lagrange-Galerkin method for the incompressible Navier-Stokes equations. SIAM J. Numer. Anal. 37 (2000) 799-826.

[2] V.I. Arnold, Ordinary Differential Equations. Springer-Verlag, Berlin, Germany (1992).

[3] S.C. Brenner and L.R. Scott, The Mathematical Theory of Finite Element Methods, Texts in Applied Mathematics 15. Springer-Verlag, New York, USA (1994).

[4] P.G. Ciarlet, Mathematical Elasticity, Vol. I: Three-Dimensional Elasticity, Studies in Mathematics and its Applications 20. North-Holland Publishing Co., Amsterdam, Netherlands (1988).

[5] P.G. Ciarlet and P.-A. Raviart, Interpolation theory over curved elements, with applications to finite element methods. Comput. Methods Appl. Mech. Engrg. 1 (1972) 217-249.

[6] J. Donea, S. Giuliani and J.P. Halleux, An arbitrary Lagrangian-Eulerian finite element method for transient dynamic fluidstructure interactions. Comput. Methods Appl. Mech. Engrg. 33 (1982) 689-723.

[7] F. Duarte, R. Gormaz and S. Natesan, Arbitrary Lagrangian-Eulerian method for Navier-Stokes equations with moving boundaries. Comput. Methods Appl. Mech. Engrg. 193 (2004) 4819-4836.

[8] C. Farhat, M. Lesoinne and N. Maman, Mixed explicit/implicit time integration of coupled aeroelastic problems: three-field formulation, geometric conservation and distributed solution. Internat. J. Numer. Methods Fluids 21 (1995) 807-835

[9] M.A. Fernández, J.-F. Gerbeau and C. Grandmont, A projection semi-implicit scheme for the coupling of an elastic structure with an incompressible fluid. Internat. J. Numer. Methods Engrg. 69 (2007) 794-821.

[10] L. Formaggia and F. Nobile, A stability analysis for the Arbitrary Lagrangian Eulerian formulation with finite elements. East-West J. Numer. Math. 7 (1999) 105-132.

[11] L. Gastaldi, A priori error estimates for the Arbitrary Lagrangian Eulerian formulation with finite elements. East-West J. Numer. Math. 9 (2001) 123-156.

[12] V. Girault, H. López and B. Maury, One time-step finite element discretization of the equation of motion of two fluid flows. Numer. Methods Partial Differ. Equ. 22 (2005) 680-707.

[13] R. Glowinski, T.-W. Pan, T.I. Hesla, D.D. Joseph and J. Periaux, A distributed Lagrange multiplier/fictitious domain method for the simulation of flow around moving rigid bodies: application to particulate flow. Comput. Methods Appl. Mech. Engrg. 184 (2000) 241-267.

[14] C. Grandmont and Y. Maday, Fluid-structure interaction: a theoretical point of view, in Fluid-structure interaction, Innov. Tech. Ser., Kogan Page Sci., London (2003) 1-22.

[15] C. Grandmont, V. Guimet and Y. Maday, Numerical analysis of some decoupling techniques for the approximation of the unsteady fluid structure interaction. Math. Models Methods Appl. Sci. 11 (2001) 1349-1377.

[16] P. Grisvard, Elliptic problems in nonsmooth domains, Monographs and Studies in Mathematics 24. Pitman (Advanced Publishing Program), Boston, USA (1985).

[17] H.H. Hu, Direct simulation of flows of solid-liquid mixtures. Int. J. Multiphase Flow 22 (1996) 335-352.

[18] T.J.R. Hughes, W.K. Liu and T.K. Zimmermann, Lagrangian-Eulerian finite element formulation for incompressible viscous flows. Comput. Methods Appl. Mech. Engrg. 29 (1981) 329-349.

[19] I. Inoue and M. Wakimoto, On existence of solutions of the Navier-Stokes equation in a time dependent domain. J. Fac. Sci. Univ. Tokyo Sect. IA Math. 24 (1977) 303-319.

[20] J. Janela, A. Lefebvre and B. Maury, A penalty method for the simulation of fluid-rigid body interaction. ESAIM: Proc. 14 (2005) 115-123.

[21] M. Lenoir, Optimal isoparametric finite elements and error estimates for domains involving curved boundaries. SIAM J. Numer. Anal. 23 (1986) 562-580.

[22] B. Maury, Characteristics ALE method for the unsteady 3D Navier-Stokes equations with a free surface. Int. J. Comput. Fluid Dyn. 6 (1996) 175-188. 
[23] B. Maury, Direct simulations of 2D fluid-particle flows in biperiodic domains. J. Comput. Phys. 156 (1999) 325-351.

[24] B. Maury and R. Glowinski, Fluid-particle flow: a symmetric formulation. C. R. Acad. Sci. Paris Sér. I Math. 324 (1997) $1079-1084$.

[25] J. Nitsche, Finite element approximations for solving the elastic problem, in Computing methods in applied sciences and engineering (Second Internat. Sympos., Versailles, 1975), Part 1, Lecture Notes in Econom. and Math. Systems 134, SpringerVerlag, Berlin, Germany (1976) 154-167.

[26] O. Pironneau, On the transport-diffusion algorithm and its applications to the Navier-Stokes equations. Numer. Math. 38 (1982) 309-332.

[27] A. Quaini and A. Quarteroni, A semi-implicit approach for fluid-structure interaction based on an algebraic fractional step method. Math. Models Methods Appl. Sci. 17 (2007) 957-983.

[28] R. Rannacher, On finite element approximation of general boundary value problems in nonlinear elasticity. Calcolo 17 (1980) $175-193$.

[29] J. San Martín, J.-F. Scheid, T. Takahashi and M. Tucsnak, Convergence of the Lagrange-Galerkin method for the equations modelling the motion of a fluid-rigid system. SIAM J. Numer. Anal. 43 (2005) 1539-1571.

[30] J. San Martín, L. Smaranda and T. Takahashi, Convergence of a finite element/ALE method for the Stokes equations in a domain depending on time. Prépublication de l'Institut Élie Cartan de Nancy 17 (2006) http://hal.archives-ouvertes.fr/hal00275223/.

[31] E. Süli, Convergence and nonlinear stability of the Lagrange-Galerkin method for the Navier-Stokes equations. Numer. Math. 53 (1988) 459-483.

[32] T. Takahashi, Analysis of strong solutions for the equations modelling the motion of a rigid-fluid system in a bounded domain. Adv. Differential Equations 8 (2003) 1499-1532. 